\title{
CALIBRATION AND CHARACTERIZATION OF CUBESAT MAGNETIC SENSORS USING A HELMHOLTZ CAGE
}

\author{
A Project \\ presented to \\ the Faculty of California Polytechnic State University, \\ San Luis Obispo
}

\author{
In Partial Fulfillment \\ of the Requirements for the Degree \\ Master of Science in Aerospace Engineering \\ by \\ Justin Dean Foley \\ December 2012
}


(C) 2012

Justin Dean Foley

ALL RIGHTS RESERVED 
TITLE:

Calibration and Characterization of CubeSat Magnetic Sensors Using a Helmholtz Cage

AUTHOR:

Justin Dean Foley

DATE SUBMITTED:

December 2012

COMMITTEE CHAIR: $\quad$ Dr. Jordi Puig-Suari

COMMITTEE MEMBER: Dr. Kira Abercromby

COMMITTEE MEMBER: Dr. Matthew Moelter

COMMITTEE MEMBER: Dr. Gerald Shaw 


\begin{abstract}
Calibration and Characterization of CubeSat Magnetic Sensors Using a Helmholtz Cage Justin Dean Foley
\end{abstract}

Small satellites, and CubeSats in particular, have quickly become a hot topic in the aerospace industry. Attitude determination is currently one of the most intense areas of development for these miniaturized systems and future Cal Poly satellite missions will depend heavily on magnetometers. In order to utilize magnetometers as a viable source of attitude knowledge, precise calibration is required to ensure the greatest accuracy achievable. This paper outlines a procedure for calibrating and testing magnetometers on the next generation of Cal Poly CubeSats, utilizing a Helmholtz cage to simulate any desired orbital magnetic field that would be experienced by a spacecraft around Earth, as well as investigation of magnetic interference as a result of on-board electrical activity.

Keywords: CubeSat, picosatellite, small satellite, Helmholtz, magnetic, magnetometer 


\section{ACKNOWLEDGMENTS}

To my family: The completion of this project has depended greatly on your consistent support. Your positive actions and words continue to provide motivation to be successful. This project is a direct result of the power that is your love and support.

To Dr. $P$ and the PolySat and CubeSat teams past and present: Thank you for giving me the opportunity to work with you doing things I enjoy every day, and for providing me with the open learning environment I appreciate and depend on. 


\section{TABLE OF CONTENTS}

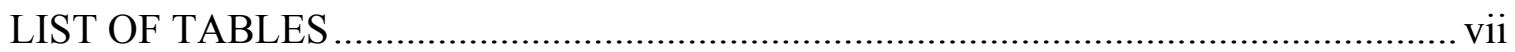

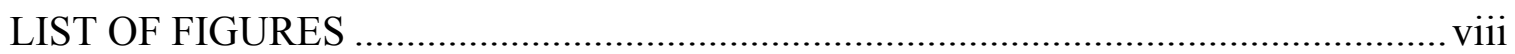

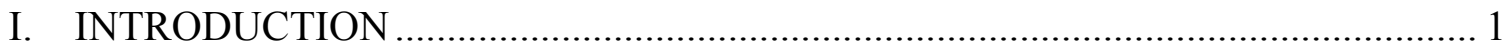

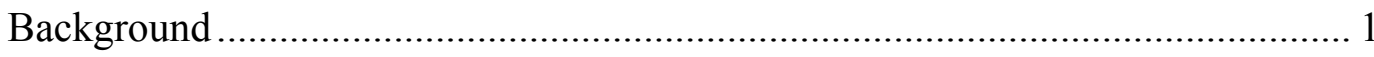

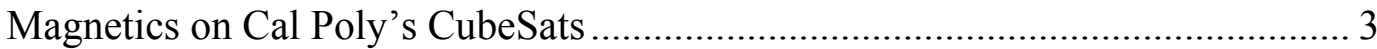

B-Dot Detumbling Algorithm........................................................................ 12

Next Generation Bus and New Magnetic Sensors................................................. 13

Statement of Problem...................................................................................... 18

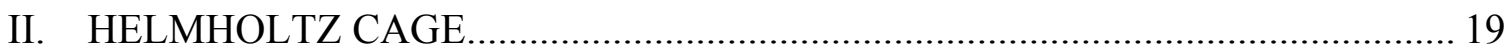

Purpose

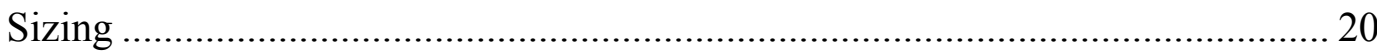

Magnetic Field Uniformity Analysis ............................................................. 22

III. CALIBRATION METHOD FOR THE MAGNETIC SENSOR ……………......... 29

Initial Testing of the HMC5883L Magnetometer................................................... 29

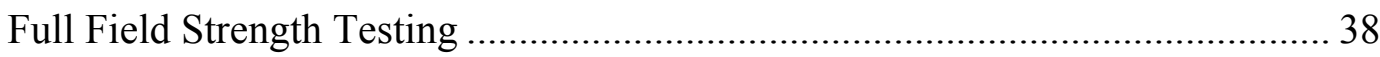

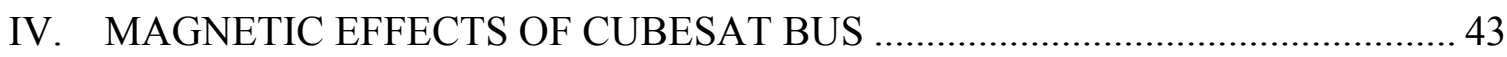

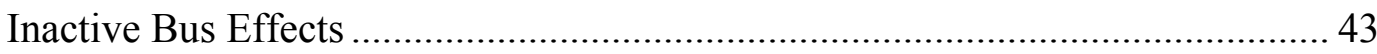

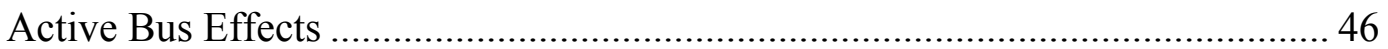

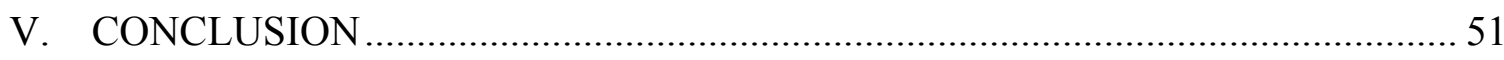

Performance of the Standalone HMC5883L....................................................... 51

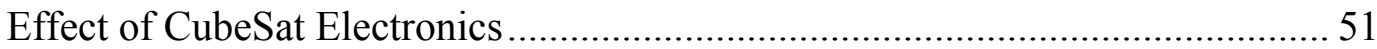

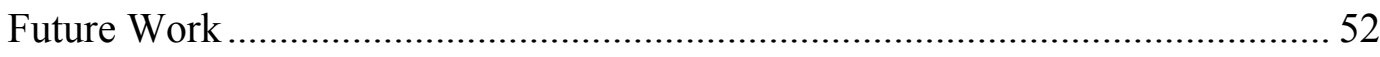

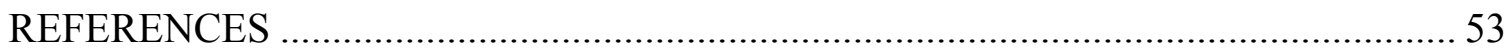

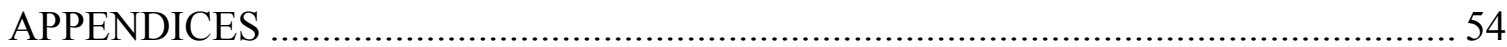

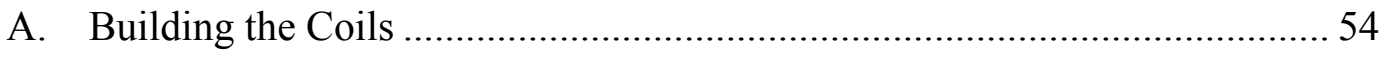

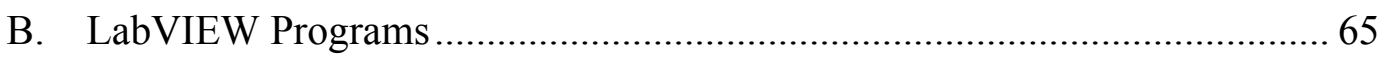

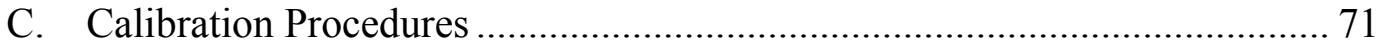




\section{LIST OF TABLES}

Table 1: Helmholtz Field Offsets at Sensor Locations ................................................ 28

Table 2: Test Setups For Each Active Bus Measurement Location ............................... 48

Table 3: Active Bus Field Strength Offsets Full Results in nT .................................... 50

Table 4: Active Bus Angle Errors Full Results in nT ................................................ 50 


\section{LIST OF FIGURES}

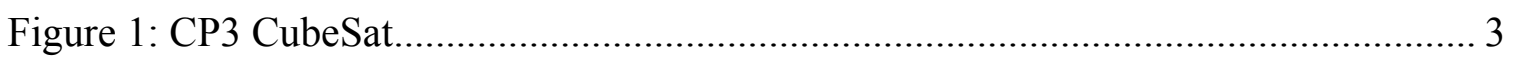

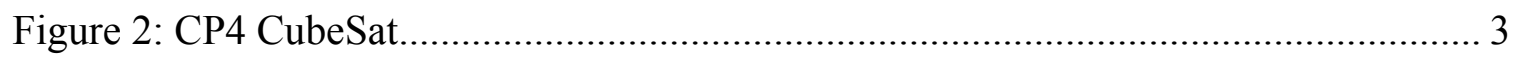

Figure 3: PolySat first generation bus........................................................................ 4

Figure 4: PolySat first generation bus expanded ....................................................... 4

Figure 5: CP3 on-orbit data, temperature vs. time .................................................. 5

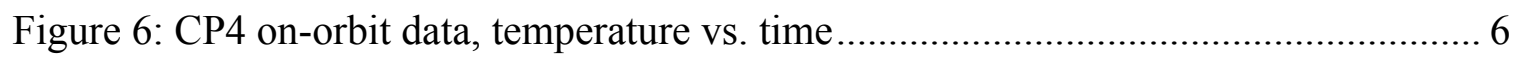

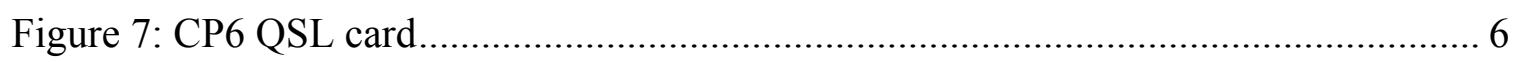

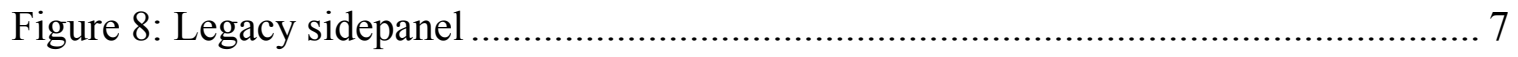

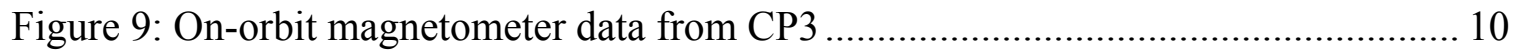

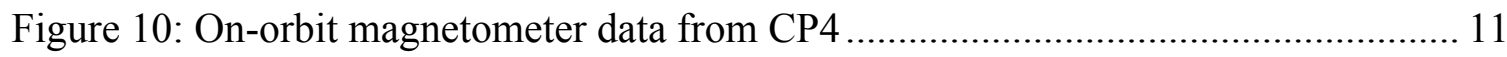

Figure 11: New Cal Poly CubeSat Bus (no batteries or side panels).............................. 14

Figure 12: IPEX (CP8) CubeSat design concept using new bus ................................. 15

Figure 13: Next Generation Sidepanel (left: full panel, right: larger view)................... 16

Figure 14: (left) Helmholtz Coils, (right) Calculated Field in Region of Coils............... 19

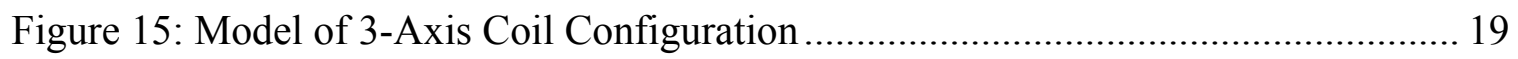

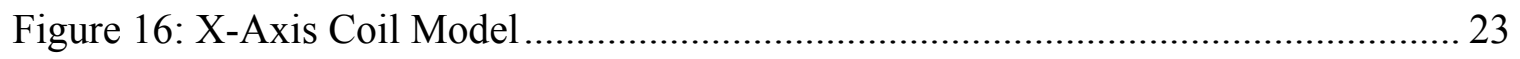

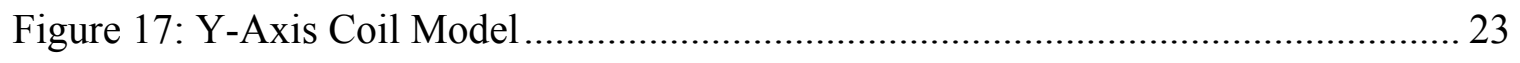

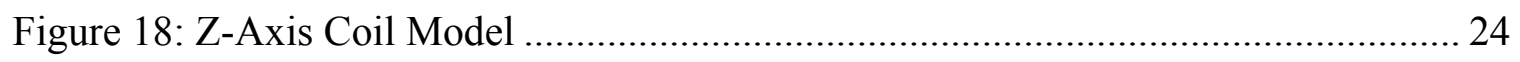

Figure 19: X-Axis Comparison of Measurements to Model ….................................... 25

Figure 20: Y-Axis Comparison of Measurements to Model ….................................... 25

Figure 21: Z-axis Comparison of Measurements to Model .......................................... 26

Figure 22: X-Axis Cross-Axis Profile from Model ...................................................... 27

Figure 23: Y-Axis Cross-Axis Profile from Model ..................................................... 27

Figure 24: Z-Axis Cross-Axis Profile from Model.................................................... 28

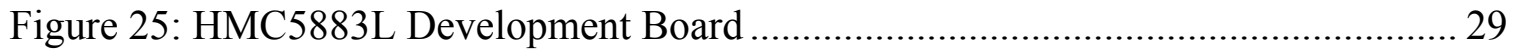




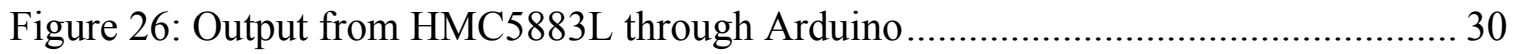

Figure 27: HMC5883L Development Board on Test Stand ......................................... 30

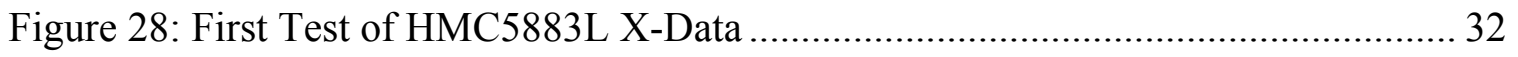

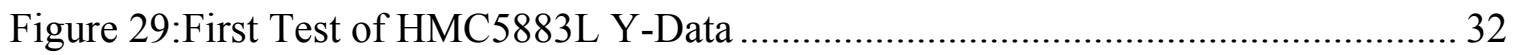

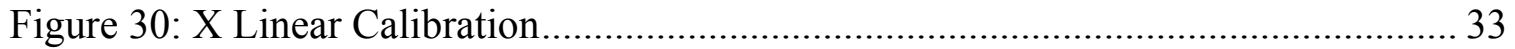

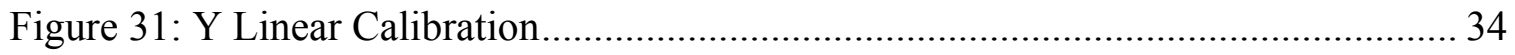

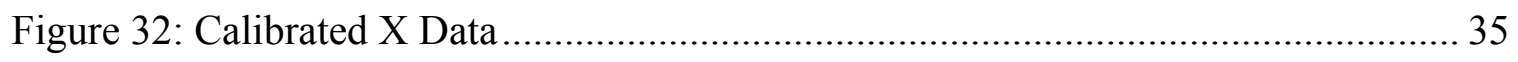

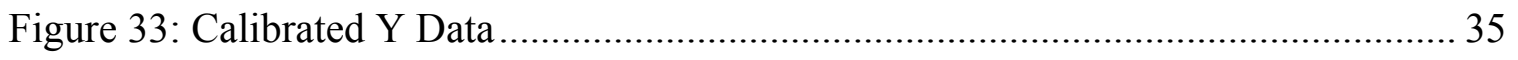

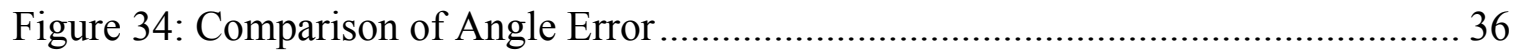

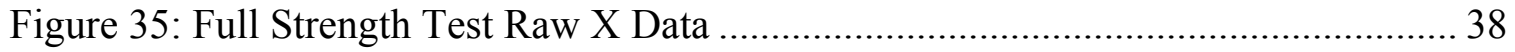

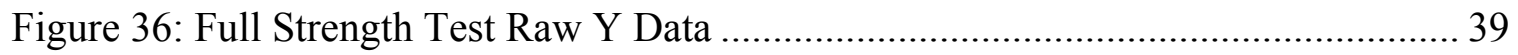

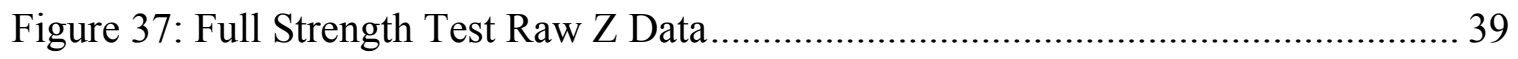

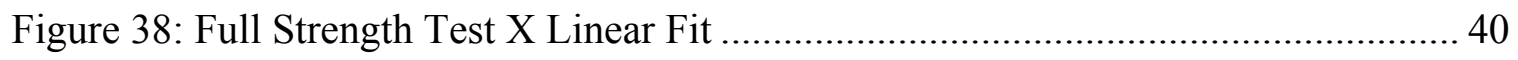

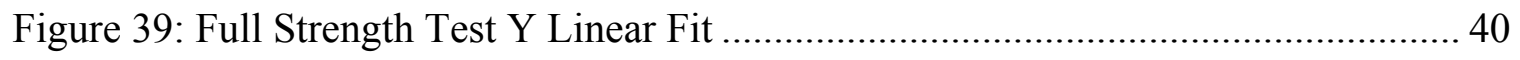

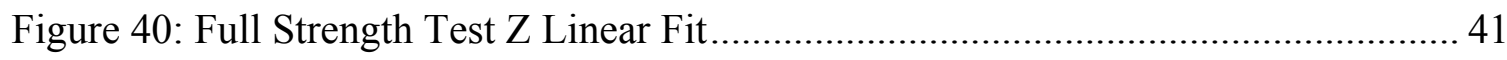

Figure 41: Full Strength Test X Calibrated............................................................... 41

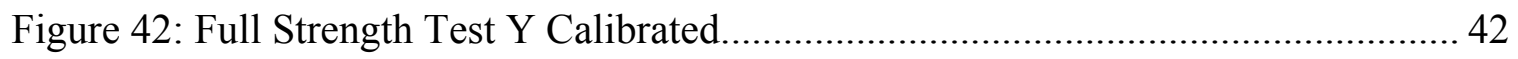

Figure 43: Full Strength Test Z Calibrated ....................................................... 42

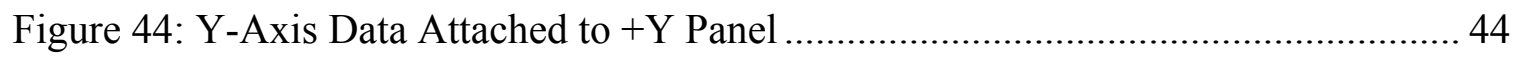

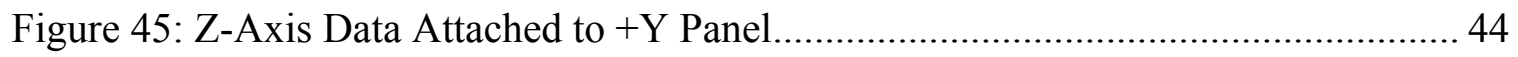

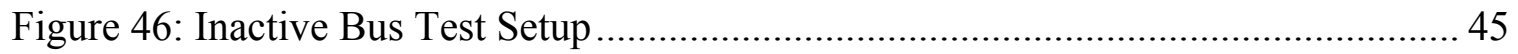

Figure 47: Active System Board Raw Field Magnitudes .......................................... 49

Figure 48: Active System Board Normalized and Aligned Field Magnitudes ................ 49 


\section{INTRODUCTION}

\section{Background}

The CubeSat standard was established in 1999 by Professor Robert Twiggs at Stanford University and Professor Jordi Puig-Suari at Cal Poly, San Luis Obispo. The goal of the standard has been to provide universities with affordable and regular access to space by establishing a standard form factor with which all entities could build to. The standard, now defined by the CubeSat Design Specification (CDS) document [1] published by the Cal Poly CubeSat program, was originally for a 10 centimeter cube no more than $1 \mathrm{~kg}$ in mass. Each 10 centimeter cube can be thought of as one CubeSat unit, or $1 \mathrm{U}$. Three $1 \mathrm{U}$ CubeSats, or any combination adding up to $3 \mathrm{U}$, will fit into the Cal Poly-built Poly Picosatellite Orbital Deployer (P-POD) which interfaces the satellites to a launch vehicle. Since the establishment of the CubeSat standard and the CDS, a multitude of CubeSats have been built and flown successfully, giving hundreds, if not thousands of students worldwide the opportunity to participate in real satellite design projects.

In addition to building the P-POD and providing launch integration services for CubeSat developers, a subset of the Cal Poly team has also been established as a wellknown CubeSat developer itself, known as PolySat. The CubeSats built by PolySat are known for making use of highly integrated systems, allowing for less volume for support electronics and more volume for payload. This is a desirable trait for CubeSats due to the high cost of launch.

As with any new technology at the forefront of electrical and mechanical potential, CubeSats have rapidly grown in complexity and capability, closely following the progress of miniaturized systems used in smartphones and portable computers. 
Power, communications, and processing capabilities have continuously improved, but one of the most sought after enablers of advanced missions on CubeSats is attitude determination, that is, the ability of the CubeSat to accurately know what direction it is pointing.

One of the most common methods of attitude determination on CubeSats is the use of magnetic sensors, usually referred to as magnetometers. The concept is nearly identical to the use of a compass to determine your direction with relation to the Earth's magnetic North pole. The needle of a compass is made of a magnetic material, and will point in the direction of the local magnetic field while balancing level to the ground. On the surface of the Earth, this field line runs more or less from South to North, providing the user a quick reference of direction in two dimensions. In space, a satellite can move freely in three dimensions and has no gravitational reference, so it is necessary to utilize a "compass" capable of reporting the magnetic field in three dimensions.

Rather than utilize a bulky needle-type magnetic measurement system, CubeSat developers, short on power and volume, take advantage of a rapidly developing market of relatively inexpensive, volume and power efficient microelectromechanical system (MEMS) sensors. Similar to MEMS acceleration and rotation rate sensors, MEMS magnetometers are extremely small, typically less than the size of a dime. 


\section{Magnetics on Cal Poly's CubeSats}

Cal Poly's first CubeSats to reach orbit, CP3 and CP4 (Figure 1 and Figure 2), share a common bus architecture. This bus, PolySat's first generation CubeSat bus, utilizes nearly identical structure, side panels, power, radio and command and data handling systems, and allows for a variety of payloads to be integrated. Examples of the basic components of the first generation bus can be seen in Figure 3 and Figure 4.

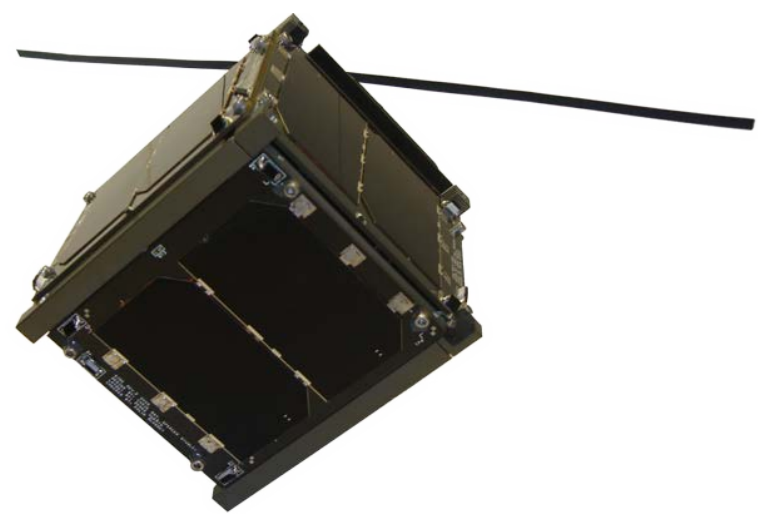

Figure 1: CP3 CubeSat

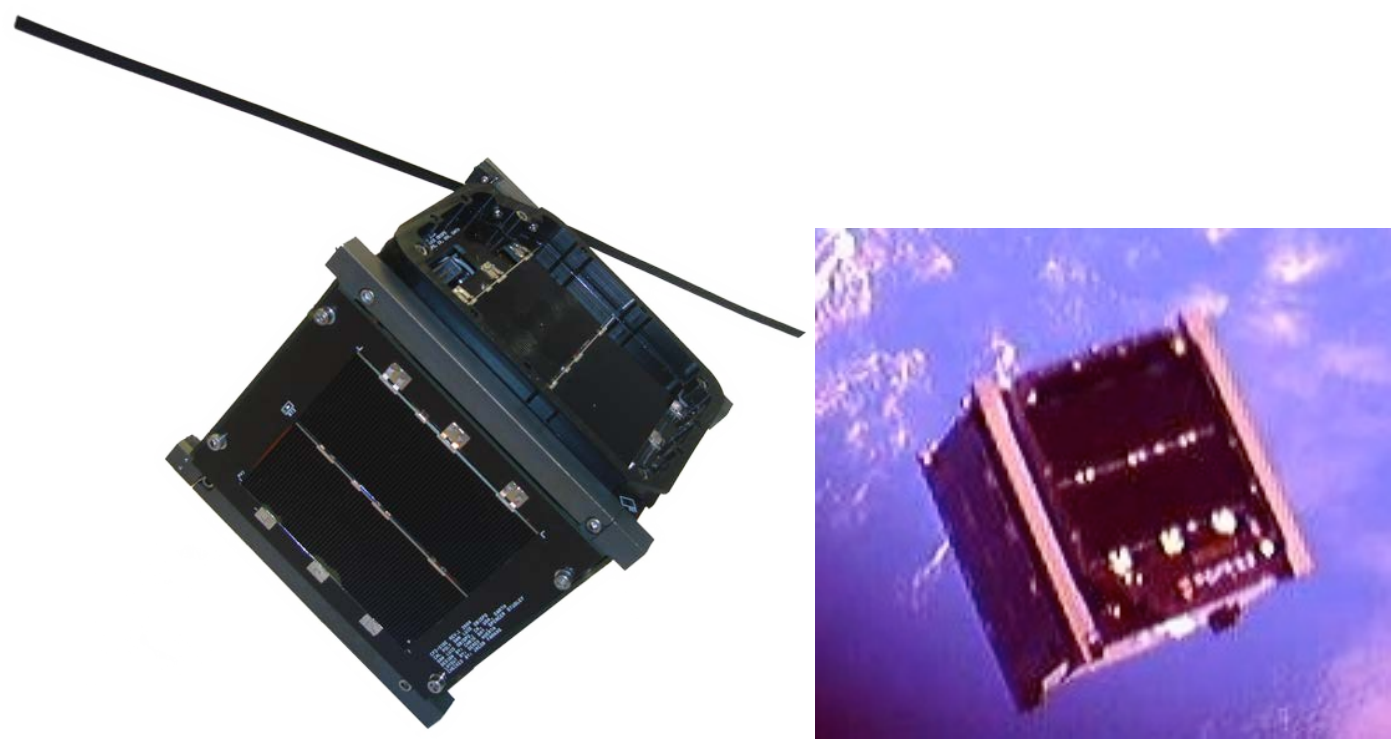

Figure 2: CP4 CubeSat 


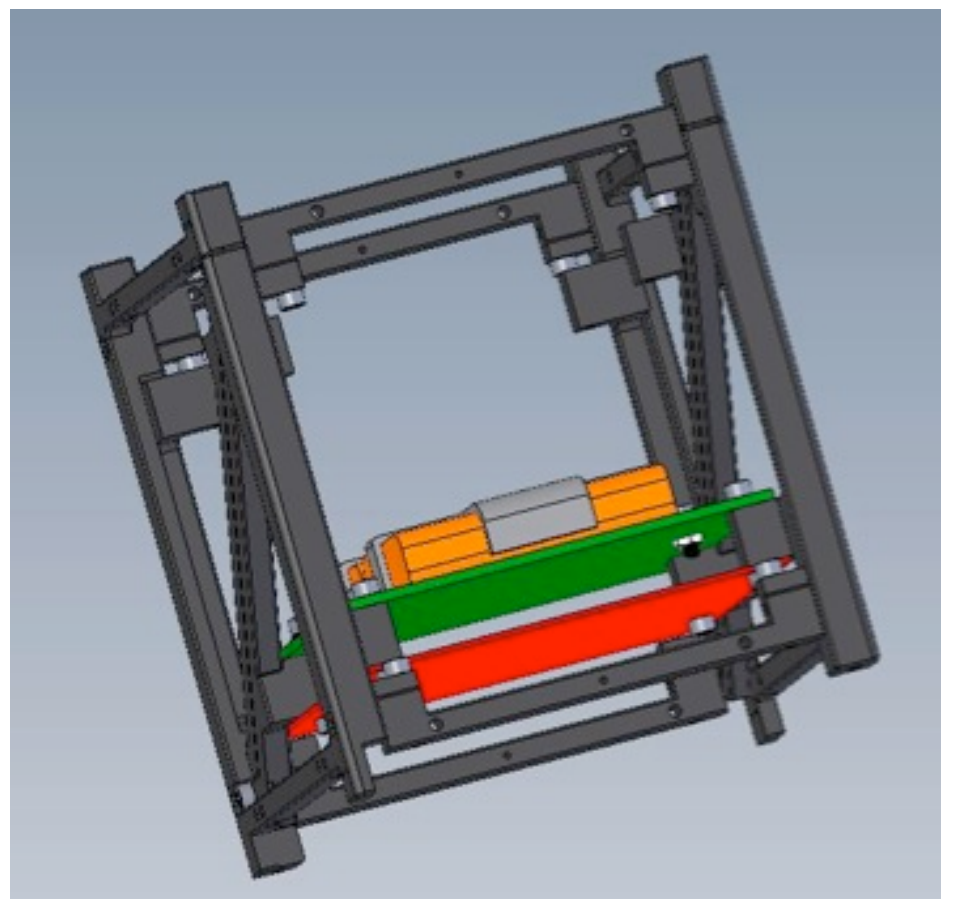

Figure 3: PolySat first generation bus

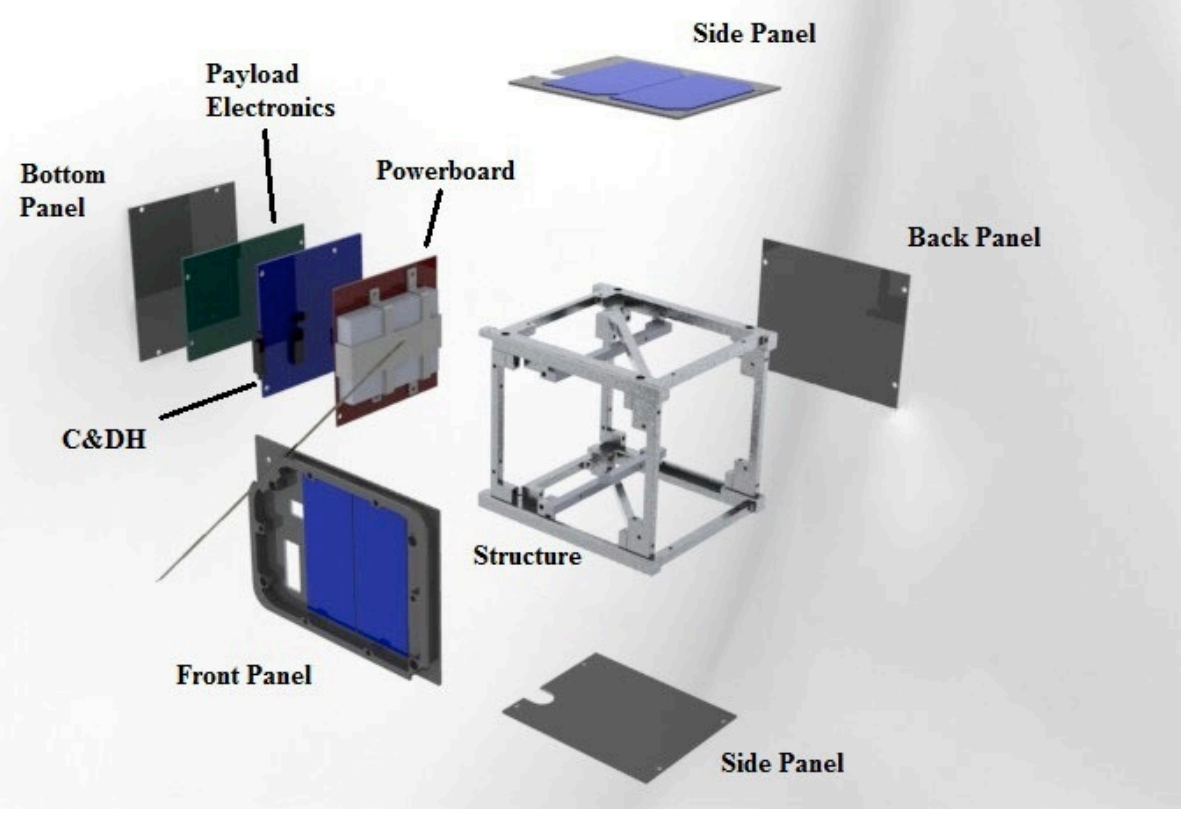

Figure 4: PolySat first generation bus expanded 
CP3 and CP4 were successfully launched together in 2007, however it was discovered shortly thereafter that both satellites suffered from poor receive sensitivity in their radios [2]. Despite the hardship, both CubeSats downlinked valuable data to the ground and provided the PolySat team with great operations and data-processing experience. While CP4 ceased functioning a few months after launch, CP3 has continued to periodically provide useful data.

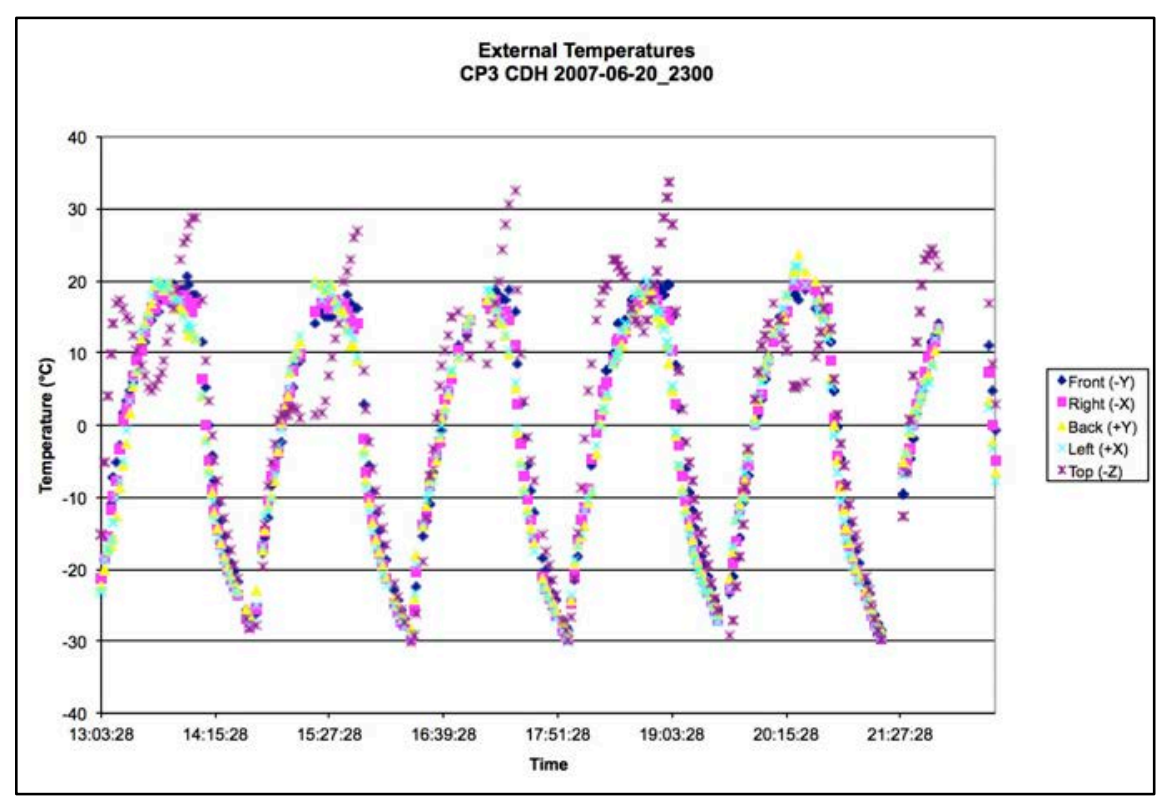

Figure 5: CP3 on-orbit data, temperature vs. time 


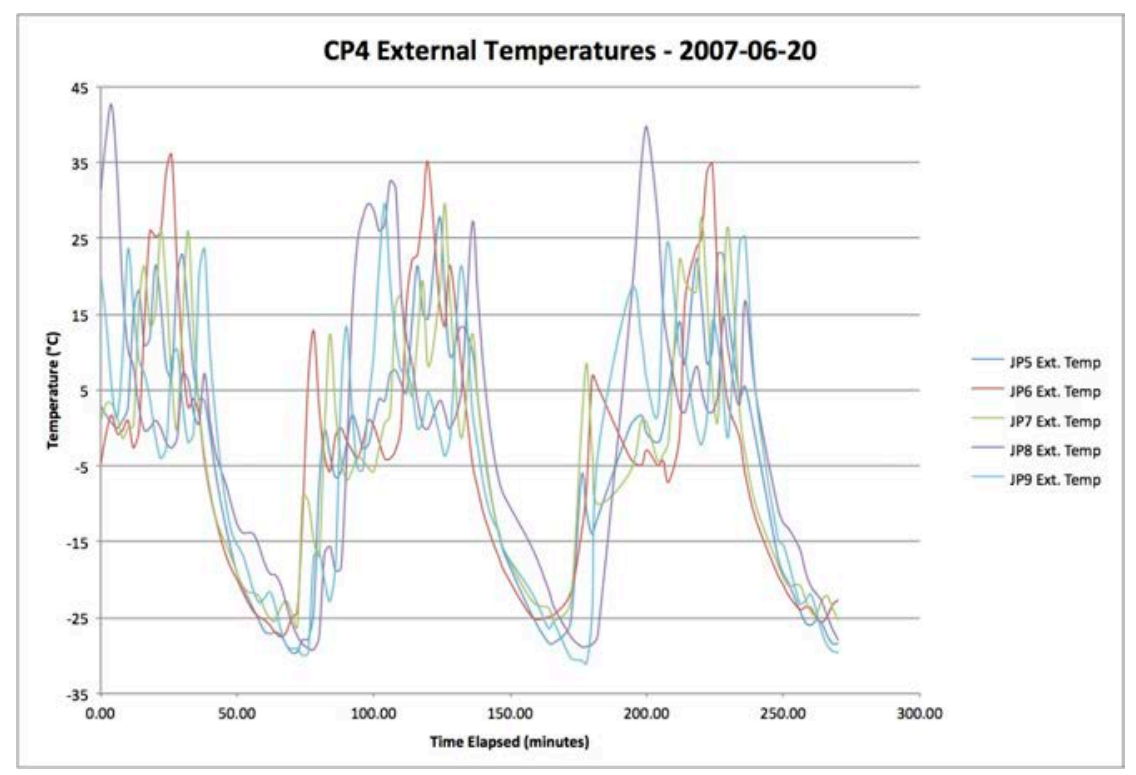

Figure 6: CP4 on-orbit data, temperature vs. time

In an attempt to salvage CP3's mission from the poor uplink ability, the PolySat team upgraded the backup CP3 flight unit with an improved radio and more advanced payload, and re-launched it as CP6 in 2008. CP6 provided even more useful data to the PolySat team before a computer lockup disabled commanding ability.

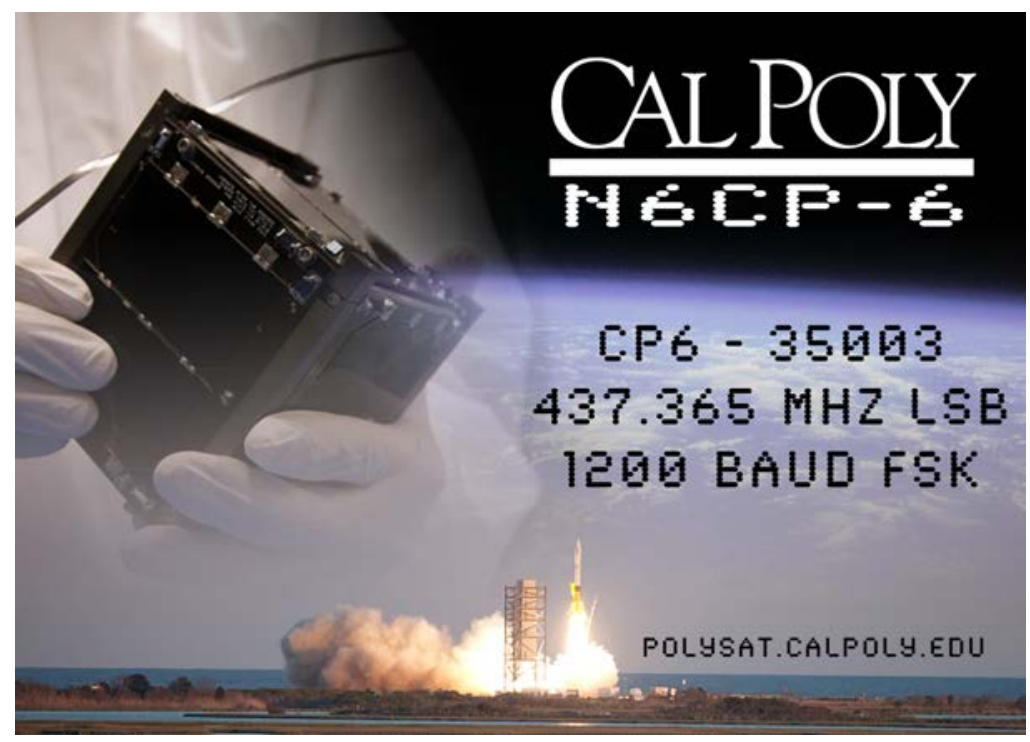

Figure 7: CP6 QSL card 
All of Cal Poly's first-generation CubeSats to successfully operate in orbit to date, CP3, CP4 and CP6, have utilized the HMC1052 2-axis magnetometer chip made by Honeywell, having one placed on each of five sides of the cube. This provides a total of ten readings, at least three independent readings for each axis. An image of the board layout showing magnetometer location is given in Figure 8.

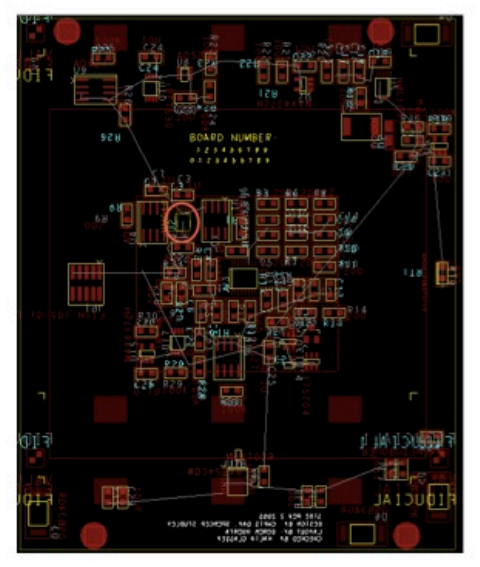

Magnetometer circled

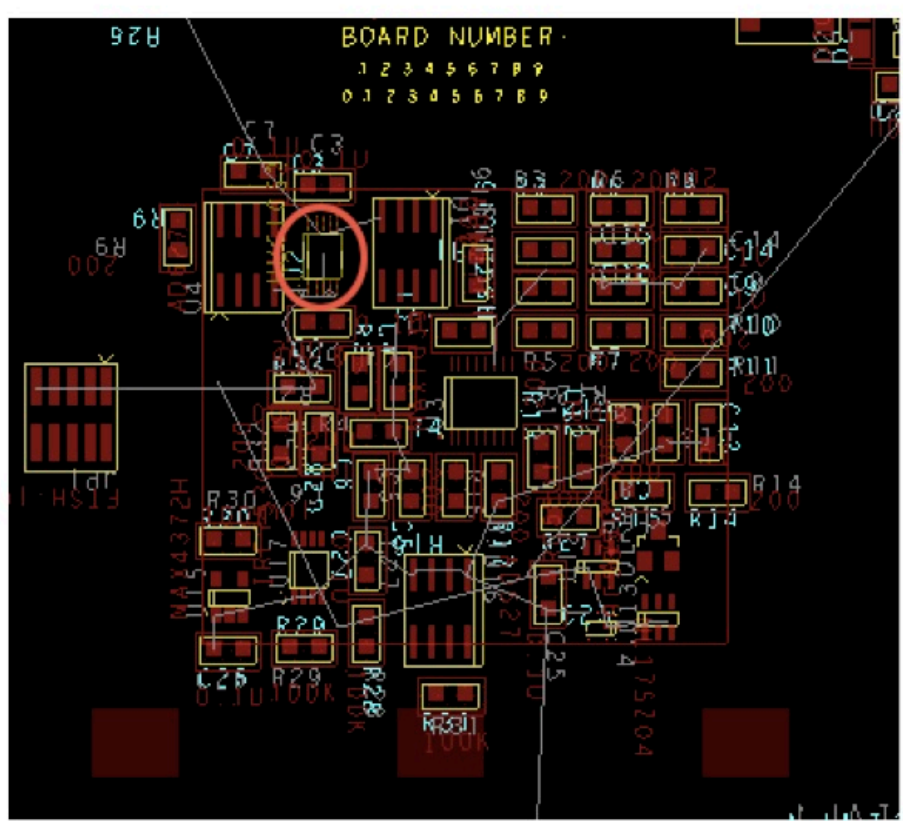

Figure 8: Legacy sidepanel

The HMC1052 outputs two analog voltage signals, one for each axis measured by the chip. These two voltages are read by an 8-bit analog to digital converter and then passed to the Command and Data Handling $(\mathrm{C} \& D H)$ processor via the satellite's $\mathrm{I}^{2} \mathrm{C}$ bus $[3]$.

Calibration of the first-generation bus magnetometers consisted of three different tests. The first two, determination of offset and gain, was performed according to documented procedures on each individual flight-candidate side panel prior to being 
chosen for integration into the satellite. The third test was an investigation of the relationship between temperature changes and magnetometer readings, though the results of this investigation were inconclusive and not formally documented.

Procedures for determining the offset and gain of the HMC1052 magnetometer are described in the Side Board testing document developed for the CP2 satellite, the first satellite in the line using the first-generation bus. The side panel is placed roughly in the center of a fixed compass rose and connected to a satellite bus connected to a lab computer. Using the debug mode of the satellite programming environment, the magnetometer analog to digital converters can be read while rotating the side panel $90^{\circ}$ at a time for offset measurement and $45^{\circ}$ at a time for gain measurement. An Excel spreadsheet was developed to assist in these tests. Based on resulting values for each angle measured, the spreadsheet provides a visual representation to confirm proper operation of the magnetometer and offers suggested values for the resistors on the side panel that control the offset and gain.

While testing of the first generation side panels verified that the magnetometer readings would not be out of range while in orbit, they did not fully characterize the relationship between the sensor readings and the body coordinate system of the satellite. The testing also did not account for offsets induced by the power system that consists of current carrying conductors embedded in and between the side panels and power board near the magnetometer chips. Interference induced by the radio transmitter, that on CP4 and CP6 operated at regular intervals due to beacons, was also not fully characterized. Examples of magnetic data downlinked from the CP3 and CP4 satellites are shown in Figure 9 and Figure 10. It is worth noting from the on-orbit data that the readings can 
vary considerably between coaxial sensors. The CP3 data was taken at a higher sensor snapshot rate and is thought to have been rotating faster than $\mathrm{CP} 4$ upon deployment from the P-POD. 

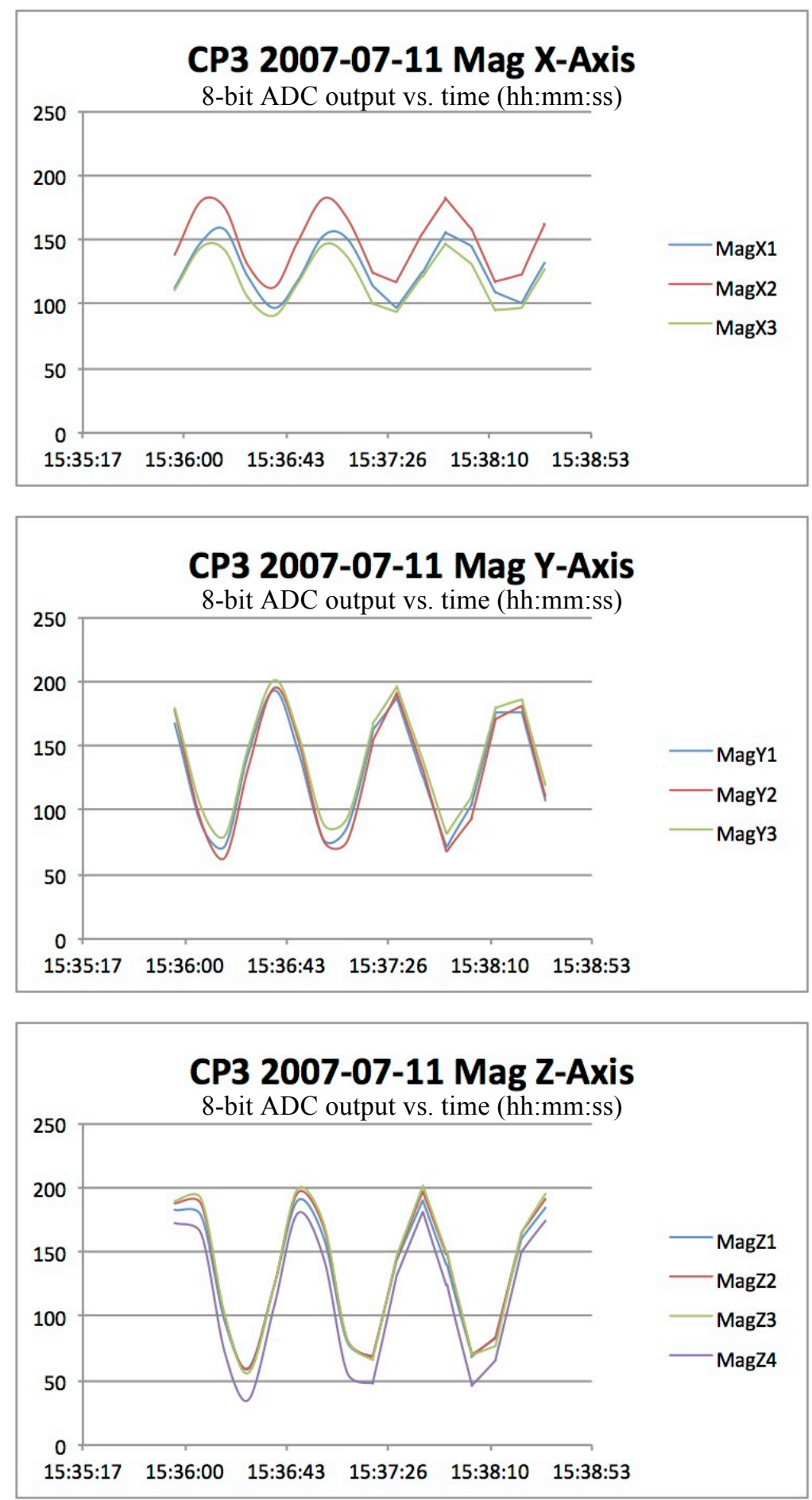

Figure 9: On-orbit magnetometer data from CP3 

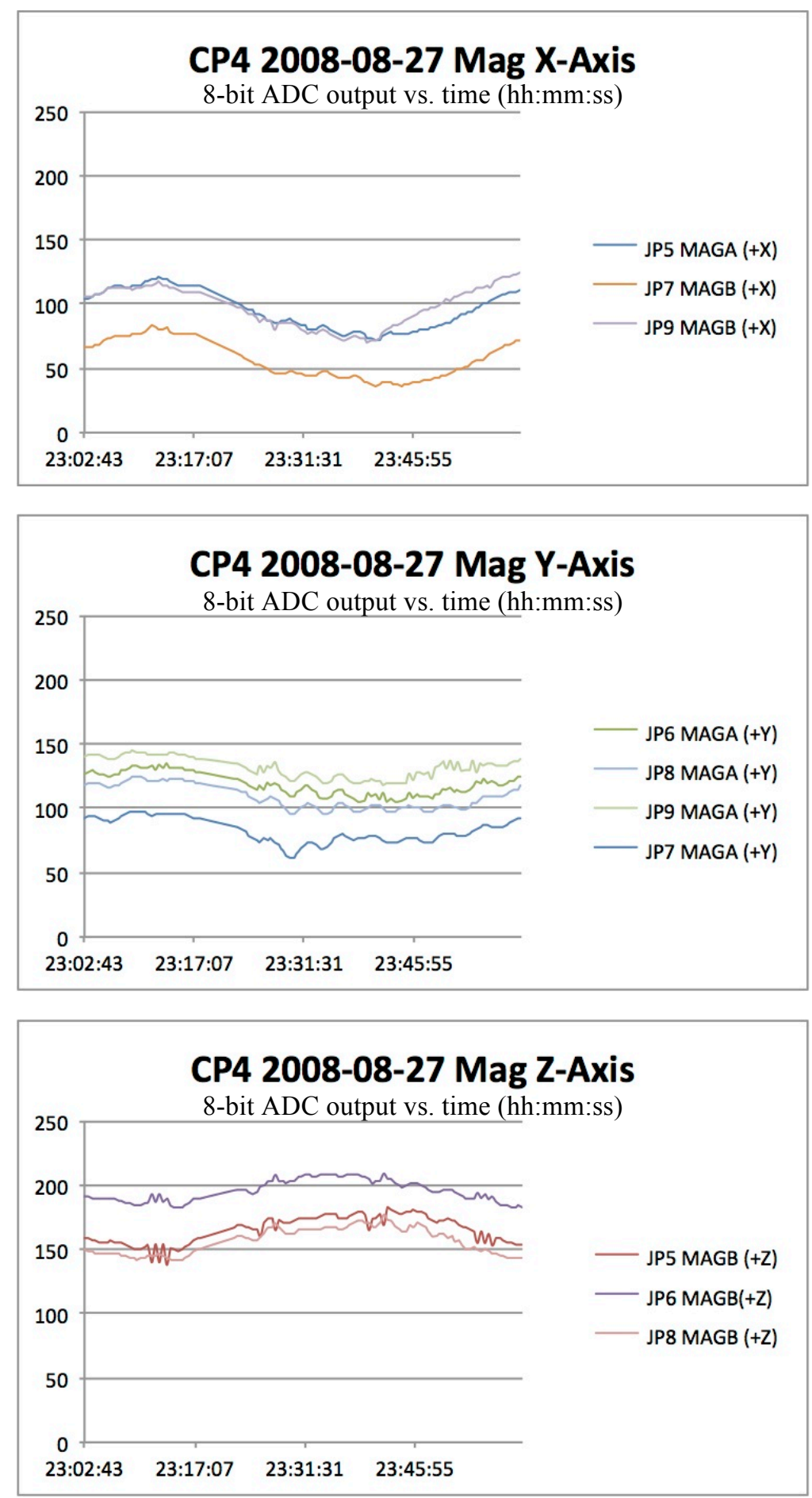

Figure 10: On-orbit magnetometer data from CP4 


\section{B-Dot Detumbling Algorithm}

While the existing calibration procedures do not fully characterize the magnetometers to the point of providing accurate true magnetic field vectors with respect to the satellite's body coordinate system, the measurements are sufficient to provide relative rotations over short time periods. This was useful for running the B-dot detumbling algorithm that uses the magnetometers combined with the magnetorquer traces built into each side panel. When run, B-dot reads the magnetometers, waits a predetermined time, reads the magnetometers again and compares the two readings. It then activates the necessary magnetorquers, in the correct direction, to counteract the rotation of the satellite. The Bdot command includes parameters to set the sign of each magnetorquer, the timing between magnetometer reads, the number of iterations, and the gain of the feedback loop [4]. B-dot was demonstrated twice on the CP6 satellite shortly before a lockup of its Command and Data Handling $(\mathrm{C} \& D H)$ processor prevented further operations. The first B-dot attempt on CP6 included an error in the command for one of the magnetorquer's signs. The result was an increase of the satellite's rotation rate. The second attempt successfully resulted in a decrease of the rotation rate of the satellite. 


\section{Next Generation Bus and New Magnetic Sensors}

The next generation Cal Poly bus is designed with the intent of pushing the envelope of highly integrated systems. The smartphone revolution has resulted in the availability of a multitude of small form factor and energy efficient electrical components that allow the next generation bus to be one of the smallest and most capable CubeSat busses to date.

The previous bus design used separate boards for Electrical Power System (EPS) and C\&DH systems, with the batteries built into the EPS board and the radio built into the C\&DH board. The new bus combines the two boards into a motherboard, but does not dictate the location of batteries. It is designed to allow mission-specific battery sizing and placement providing flexibility for capacity, thermal management and center of gravity location. The new bus also separates the radio from the C\&DH and instead uses a closely integrated daughterboard concept. The radio daughterboard helps to decouple electrical and RF noise between the motherboard and the radio, and allows for different radio band options without a complete motherboard redesign. The current radio design for the new bus uses UHF frequencies and has data throughput capability in the 10's or 100's of kbps. The old bus was never developed past $1.2 \mathrm{kbps}$. The basic components of the next generation Cal Poly bus are shown in Figure 11. 


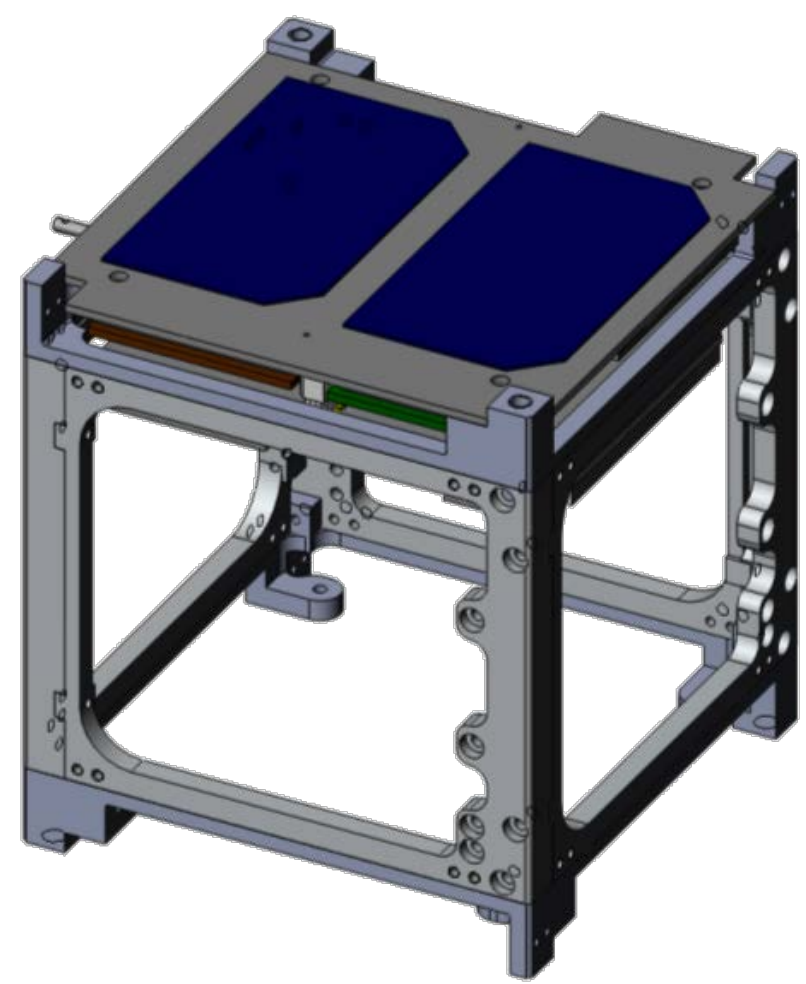

Figure 11: New Cal Poly CubeSat Bus (no batteries or side panels)

The side panels used in the old bus all connected to the EPS where solar generated power could pass more directly to the batteries. Data was piped from the side panels through the EPS board to the C\&DH board via a board-to-board interboard connector. In the new bus, the side panels all connect to the top panel through thin ribbon-type cables used in modern portable electronics. The top panel is connected directly to the motherboard. This design keeps the amount of hardware necessary for side panel connection minimal while simplifying the steps needed to assemble and disassemble the satellite. The IPEX CubeSat shown in Figure 12 is one of the first CubeSats to make use of the new bus design. 


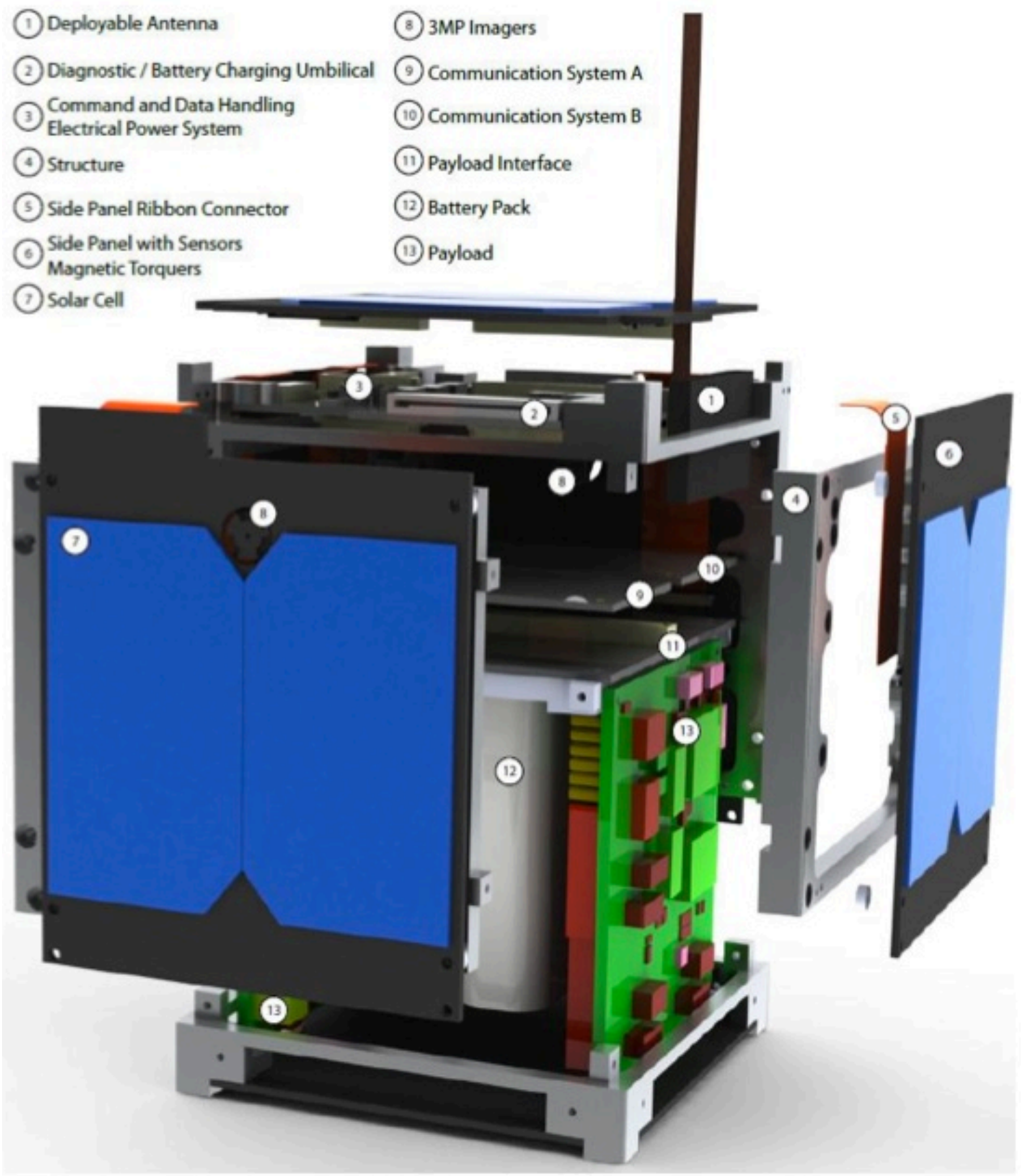

Figure 12: IPEX (CP8) CubeSat design concept using new bus 
As is the case with the legacy bus design, the new bus uses magnetometers on each of the side panels as shown in Figure 13. The new magnetometer module is the HMC5883L, which provides a three-axis, 12-bit digital output without the need for external support electronics [5]. In addition to placing one of these modules on each side panel, the new bus motherboard contains an additional HMC5883L. This results in a potential of seven independent three-axis magnetometer readings: one on each of the six sides plus one on the motherboard. Since some missions require the use of a whole side of the CubeSat dedicated to payload, this ensures that redundant magnetic readings will be available regardless of side panel configuration.
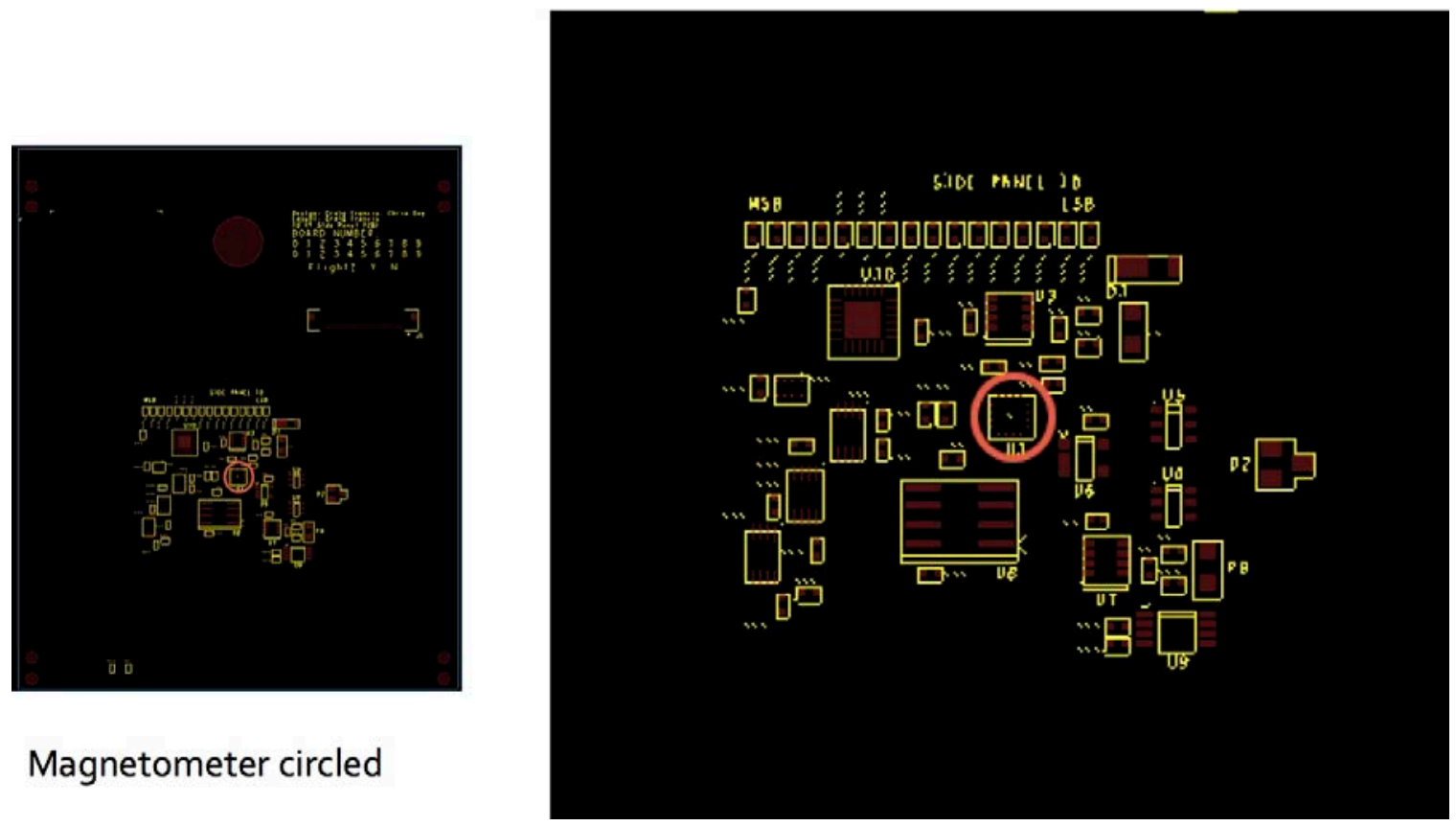

Magnetometer circled

Figure 13: Next Generation Sidepanel (left: full panel, right: larger view)

Both the HMC1052 and the HMC5883L modules utilize Honeywell's Anisotropic Magnetoresistive (AMR) technology to make magnetic field measurements. AMR is a property of a material where its resistance is effected by the relative direction of the 
magnetic field it is experiencing. The resistance is measured and amplified. In the case of the HMC5883L, the analog signal is converted to a digital value by a built-in analog to digital converter [6]. Magnetoresistive effects are also commonly utilized in the construction of hard disk drives and MEMS vibration sensors. 


\section{Statement of Problem}

The goal of the calibration process is to characterize the magnetic sensors so the satellite can accurately read magnetic field strength and direction under different modes of operation. This entails subjecting the sensors to well-known magnetic fields, comparing the reported values to the actual ones and making note of any offsets that may result from manufacturing or on-board noise. The offsets can then be subtracted from the reported values to produce accurate readings.

To generate a well-known field, a Helmholtz cage will be used consisting of three orthogonal pairs of electromagnetic coils. The article under test can be placed inside the Helmholtz cage, and by applying a computer-controlled current supply to the coils in the correct manner, can simulate changes and rotations of magnetic field while leaving the test article stationary.

The Helmholtz cage will pre-calibrated with a vector magnetometer. The vector magnetometer is a piece of lab equipment that also must be calibrated. That calibration is done by the manufacturer of the magnetometer, and must be performed prior to testing in order to provide the most accurate results. The following procedure can be followed for practice purposes without calibrating the vector magnetometer, but when it comes time to characterize a flight CubeSat it will need to be sent off for calibration. 


\section{HELMHOLTZ CAGE}

\section{Purpose}

The purpose of the Helmholtz cage is to create magnetic fields. Helmholtz coils are commonly used because of ease of construction and ability to create an essentially uniform field inside. By definition, it has two coaxial, circular coils of wire connected in series and separated by a distance equal to the radius of the coils as shown in Figure 14.

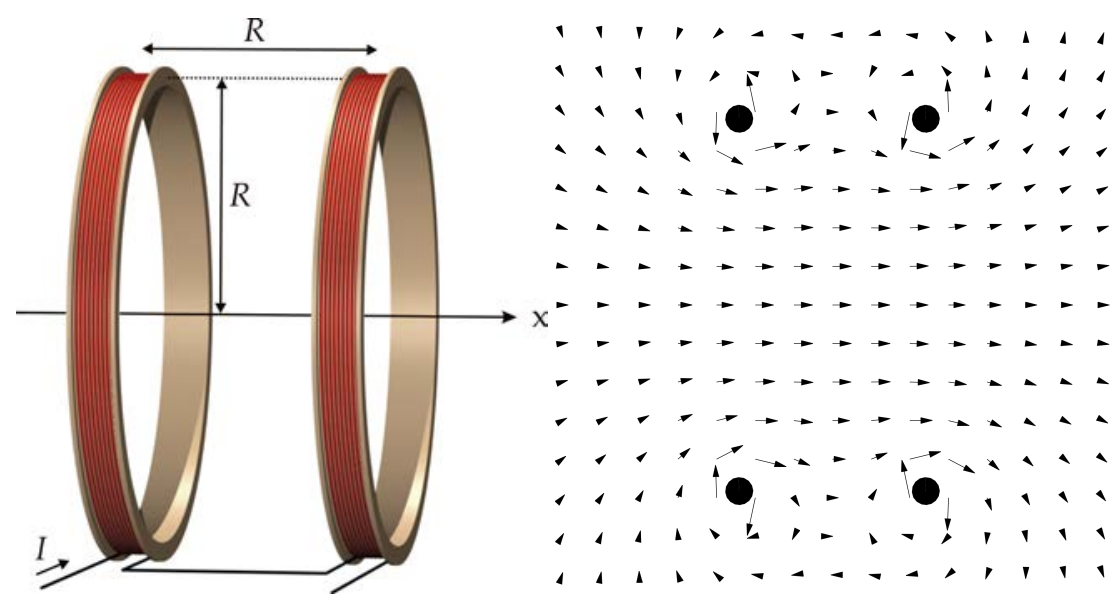

Figure 14: (left) Helmholtz Coils, (right) Calculated Field in Region of Coils

For this application, three orthogonal coils are used for full, three-dimensional control, with the smallest coil size being based on the desired testing volume.

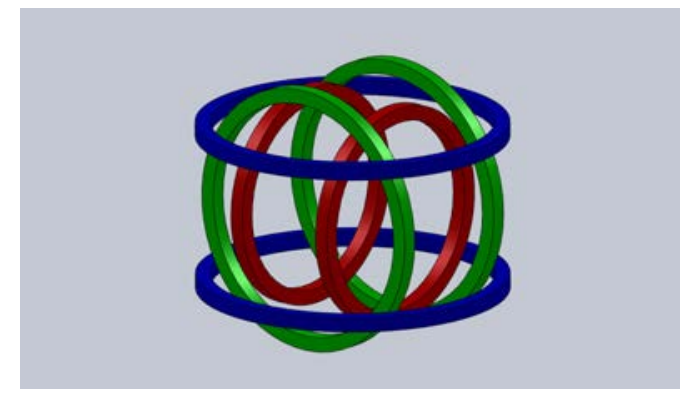

Figure 15: Model of 3-Axis Coil Configuration 


\section{Sizing}

To determine the sizes of the two larger coils, the following equation can be used:

$$
D=\sqrt{2} \sqrt{\left(\frac{d}{2}+t\right)^{2}+\left(\frac{d}{2}+2 w\right)^{2}}
$$

where $\mathrm{d}$ is the inner diameter of the smaller coil, $\mathrm{D}$ is the inner diameter of the larger coil, $\mathrm{t}$ is thickness and $\mathrm{w}$ is width. This equation is used to go from the small coil to the medium coil, and again from the medium coil to the large coil.

The original design of the Helmholtz cage was intended to be used around a 12inch spherical air bearing while remaining small enough to use on a small workbench. Thus the smallest coil diameter was chosen to be 13 inches, and the width and thickness of the plastic housing chosen to hold the wire is one inch. Using the previous equation, the medium coil would have an inner diameter of 16 inches and the large coil would have an inner diameter of 19 inches.

Starting with the desired dimension based on the radius/coil separation $(\mathrm{R})$ and magnetic field (B), the required current (I) and number of turns (n) can be traded and selected according to the following equation for field strength at the center of a Helmholtz coil:

$$
B=\left(\frac{4}{5}\right)^{3 / 2} \frac{\mu_{0} n I}{R}
$$

The power supply for driving the coils must also be taken into consideration when calculating the amount and type of wire to be used to ensure it can provide the current needed to create the desired magnetic field. The power supply available for this project 
can provide up to $1 \mathrm{~A}$ of current simultaneously for all outputs, and the desired capability of the cage is to cancel the local field plus generate a new field on top of it, essentially doubling the required field generating capability. The $\mathrm{X}$ and $\mathrm{Y}$ coils are in the horizontal field directions and the $\mathrm{Z}$ coil is in the vertical field direction. In San Luis Obispo, the horizontal component of the Earth's magnetic field is $\sim 0.25$ Gauss and the vertical component is $\sim 0.35$ Gauss. The values 0.25 Gauss and 0.35 Gauss are converted to Teslas for use in calculation as $2.5 \times 10^{-5} \mathrm{~T}$ and $3.5 \times 10^{-5} \mathrm{~T}$ accordingly, and doubled to account for full range, $5 \times 10^{-5} \mathrm{~T}$ for $\mathrm{X}$ and $\mathrm{Y}$, and $7 \times 10^{-5} \mathrm{~T}$ for $\mathrm{Z}$. Therefore, the following information is used to calculate the required number of turns in each coil.

\begin{tabular}{|c|c|c|}
\hline \multicolumn{3}{|c|}{ X-Coil (horizontal) } \\
\hline Diameter= & 19 & in \\
\hline Current $(\mathrm{I})=$ & 1 & $A$ \\
\hline Field Magnitude $=$ & $5.00 \mathrm{E}-05$ & $\mathrm{~T}$ \\
\hline Radius $(m)=$ & 0.2413 & $\mathrm{~m}$ \\
\hline$\mu_{0}=$ & $1.26 \mathrm{E}-06$ & $\mathrm{~T} * \mathrm{~m} / \mathrm{A}$ \\
\hline Number of turns $(n)=$ & 13.4 & turns \\
\hline \multicolumn{3}{|c|}{ Y-Coil (horizontal) } \\
\hline Diameter= & 16 & in \\
\hline Current $(\mathrm{I})=$ & 1 & $A$ \\
\hline Field Magnitude $=$ & $5.00 E-05$ & $\mathrm{~T}$ \\
\hline Radius $(m)=$ & 0.2032 & $\mathrm{~m}$ \\
\hline$\mu_{0}=$ & $1.26 \mathrm{E}-06$ & $\mathrm{~T}^{*} \mathrm{~m} / \mathrm{A}$ \\
\hline Number of turns $(n)=$ & 11.3 & turns \\
\hline \multicolumn{3}{|c|}{ Z-Coil (vertical) } \\
\hline Diameter = & 13 & in \\
\hline Current $(\mathrm{I})=$ & 1 & $A$ \\
\hline Field Magnitude $=$ & $7.00 \mathrm{E}-05$ & $\mathrm{~T}$ \\
\hline $\operatorname{Radius}(\mathrm{m})=$ & 0.1651 & $\mathrm{~m}$ \\
\hline$\mu_{0}=$ & $1.26 \mathrm{E}-06$ & $\mathrm{~T}^{*} \mathrm{~m} / \mathrm{A}$ \\
\hline Number of turns $(n)=$ & 12.8 & turns \\
\hline
\end{tabular}

For added margin, each coil was given 20 turns of wire. 


\section{Magnetic Field Uniformity Analysis}

Although the field inside a Helmholtz cage is relatively uniform near the center, it is important to note that there is non-uniformity away from the center and near the coils. This non-uniformity must be analyzed to ensure it does not introduce more inaccuracy than desired. In addition, due to the imperfections resulting from the "do it yourself" nature of this cage design, each set of coils must be modeled and mapped based on asbuilt dimensions. The location of the magnetic sensors in the Cal Poly bus provide an advantage in that they are located on the faces of a $10 \mathrm{~cm}$ cube. Because any nonuniformity of the magnetic field of the Helmholtz cage is symmetrical, the CubeSat can be placed in the center of the cage and any non-uniformity can be easily subtracted. In addition, the field inside the cage can be mathematically generated using a finite element modeling application based on as-built dimensions and compared with actual measurements taken with the vector magnetometer to produce a theoretical best achievable accuracy. The software package chosen is Finite Element Method Magnetics, or FEMM [7]. Finite element models of the theoretical magnetic fields are shown in Figure 16, Figure 17, and Figure 18. These models are based on as-built dimensions and properties of the actual Helmholtz coils and assume a 0.5 amp current is running through the conductors to produce the magnetic field strengths. The finite element modeling application uses an axisymmetric solving method so each model represents half of each respective coil. The coils are going through the page and represented by small black squares. The area occupied by the CubeSat is drawn on each figure and lies at the center of each coil. Each field map represents approximately 18 " on each side and arrows are spaced at 1" intervals. The color represents the field intensity. 


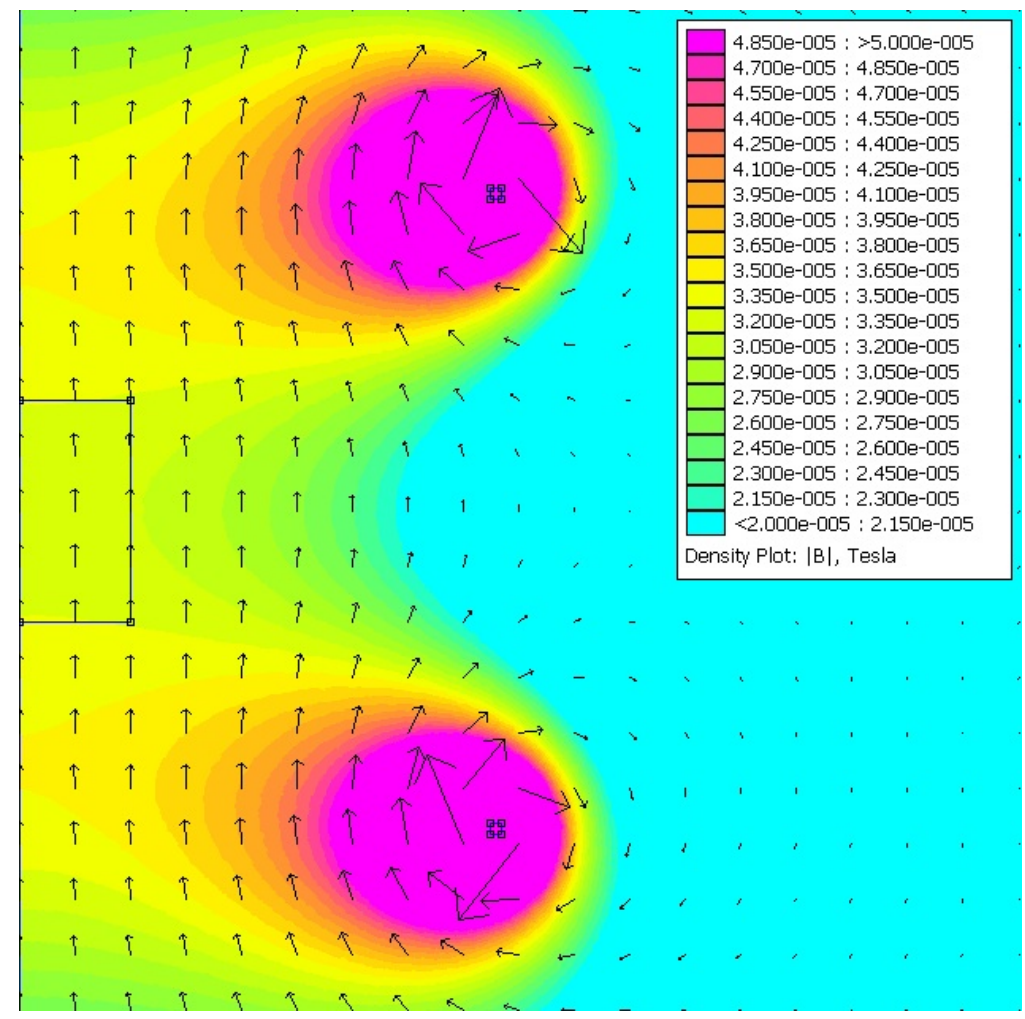

Figure 16: X-Axis Coil Model

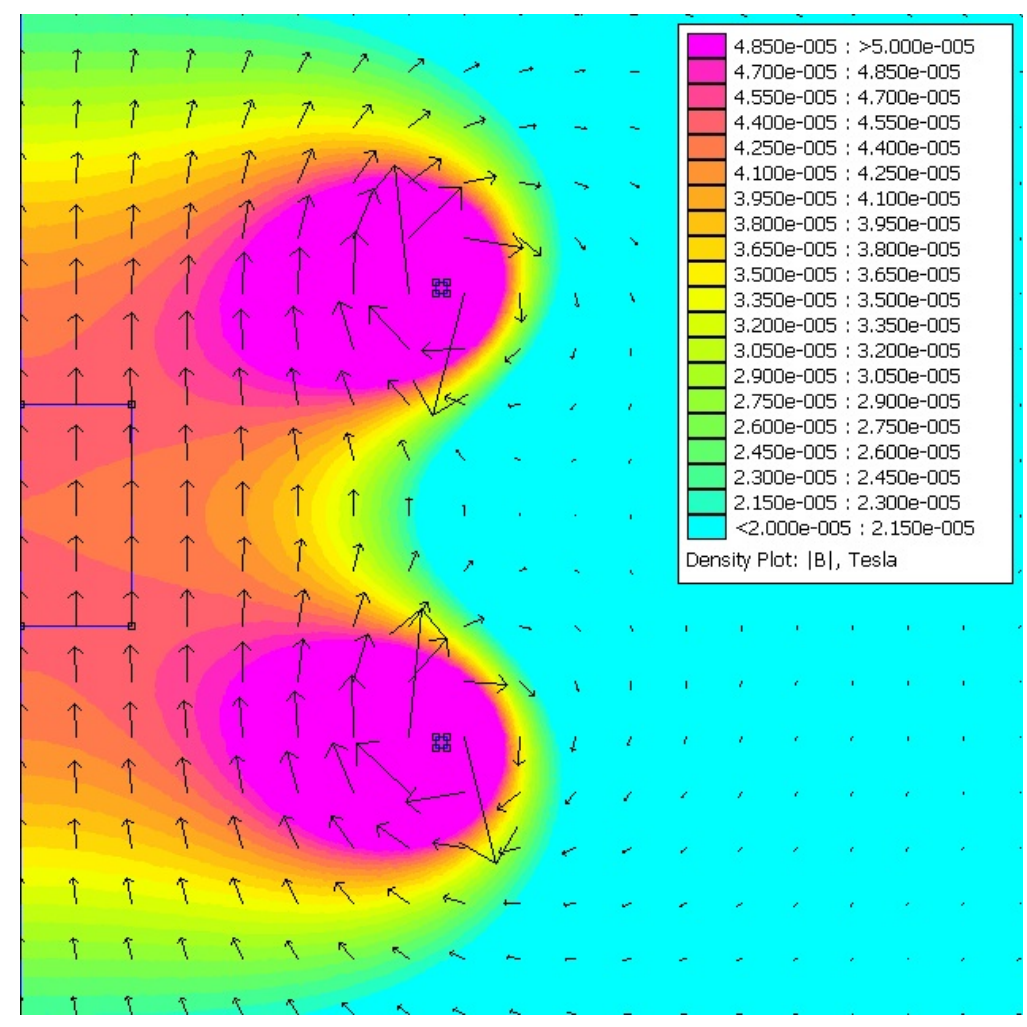

Figure 17: Y-Axis Coil Model 


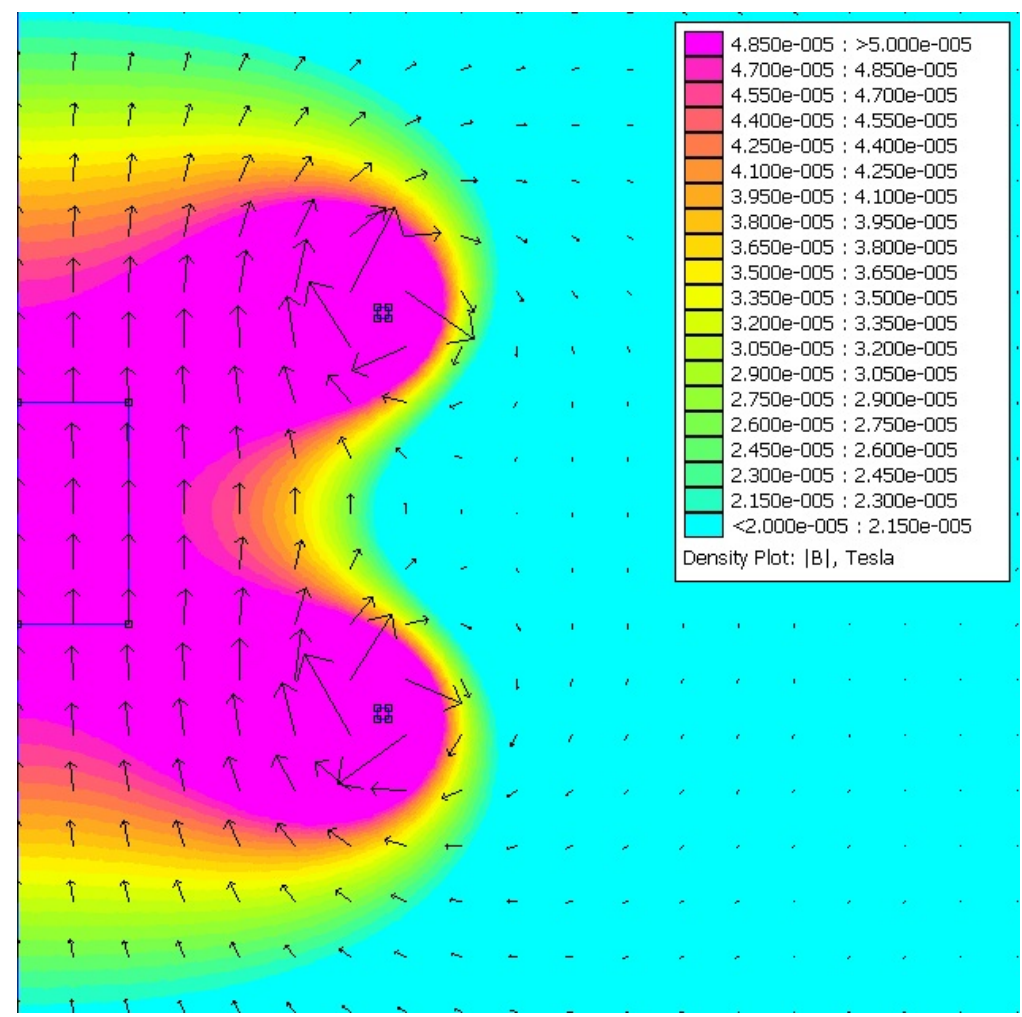

Figure 18: Z-Axis Coil Model

To confirm the reliability of the finite element models, measurements were taken along the center axis of each coil and compared to a plot of the same locations in the model. In all cases the curve shape is nearly identical and the field value is accurate to within $6 \%$ or better. The field offset is attributed to imperfections in the physical design and build of the coils. Calculating the difference and applying it as an offset can reduce the error to less than $1 \%$ in the measured regions. In Figure 19, Figure 20, and Figure 21 below, the actual field as measured by the vector magnetometer is compared to the "FEMM" software finite element model output. The "FEMM+" curve represents the finite element output with an offset to match the actual field readings to compare the curve shape. 


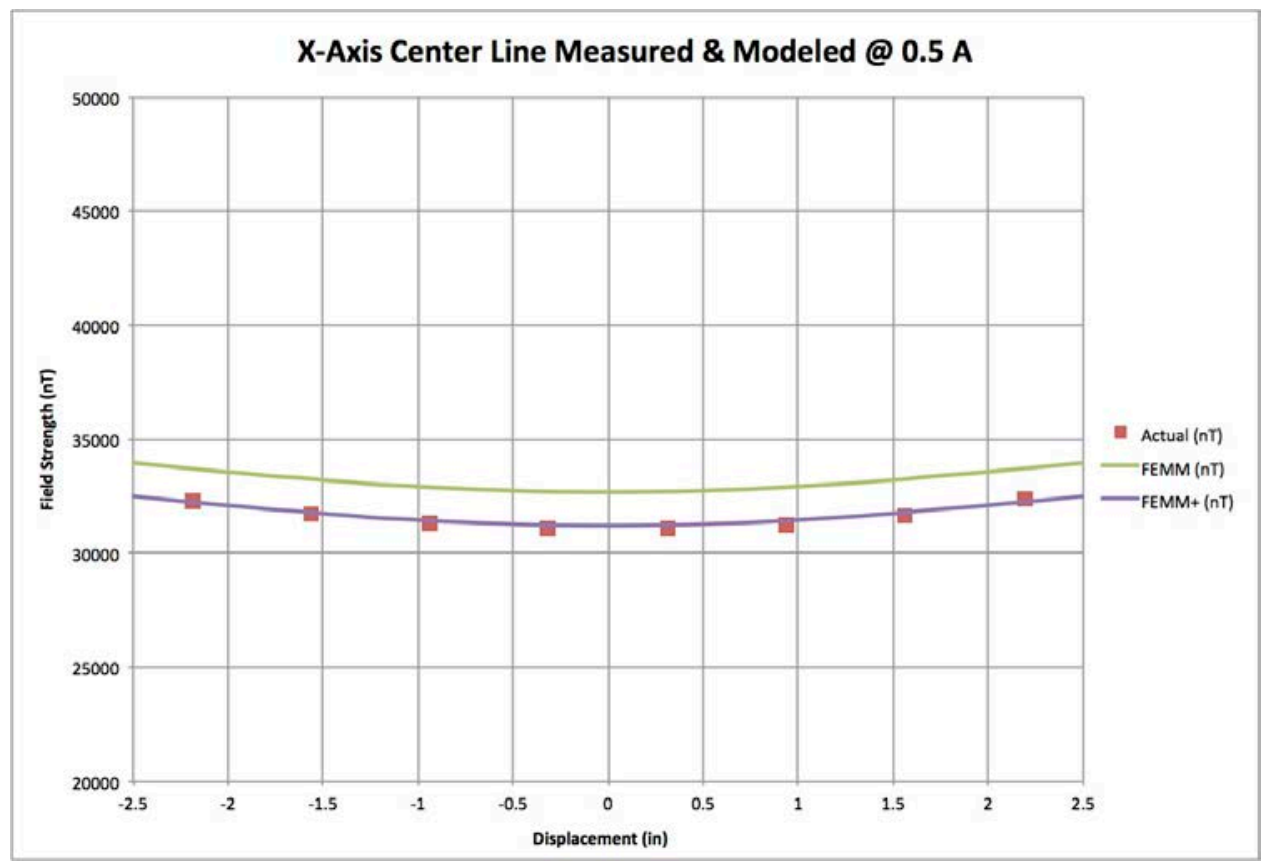

Figure 19: X-Axis Comparison of Measurements to Model

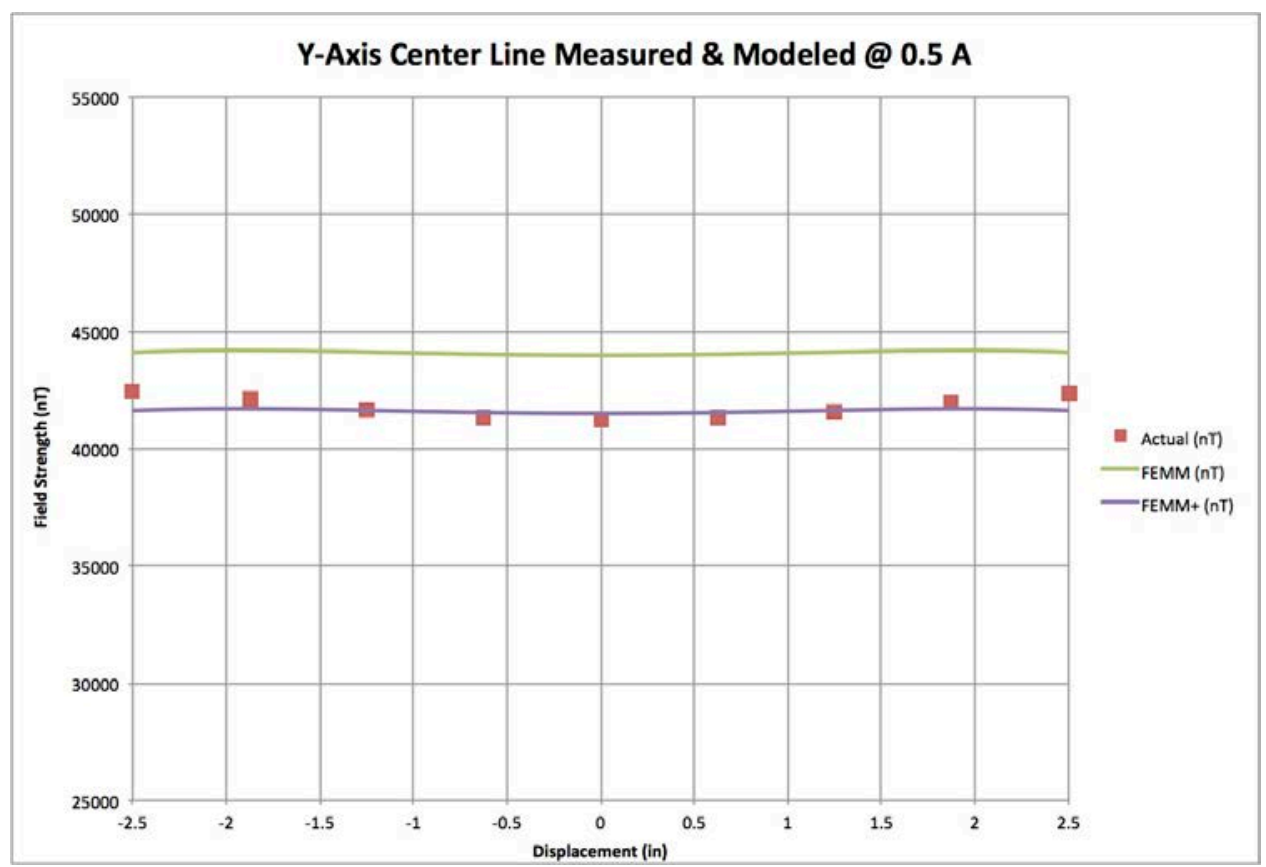

Figure 20: Y-Axis Comparison of Measurements to Model 


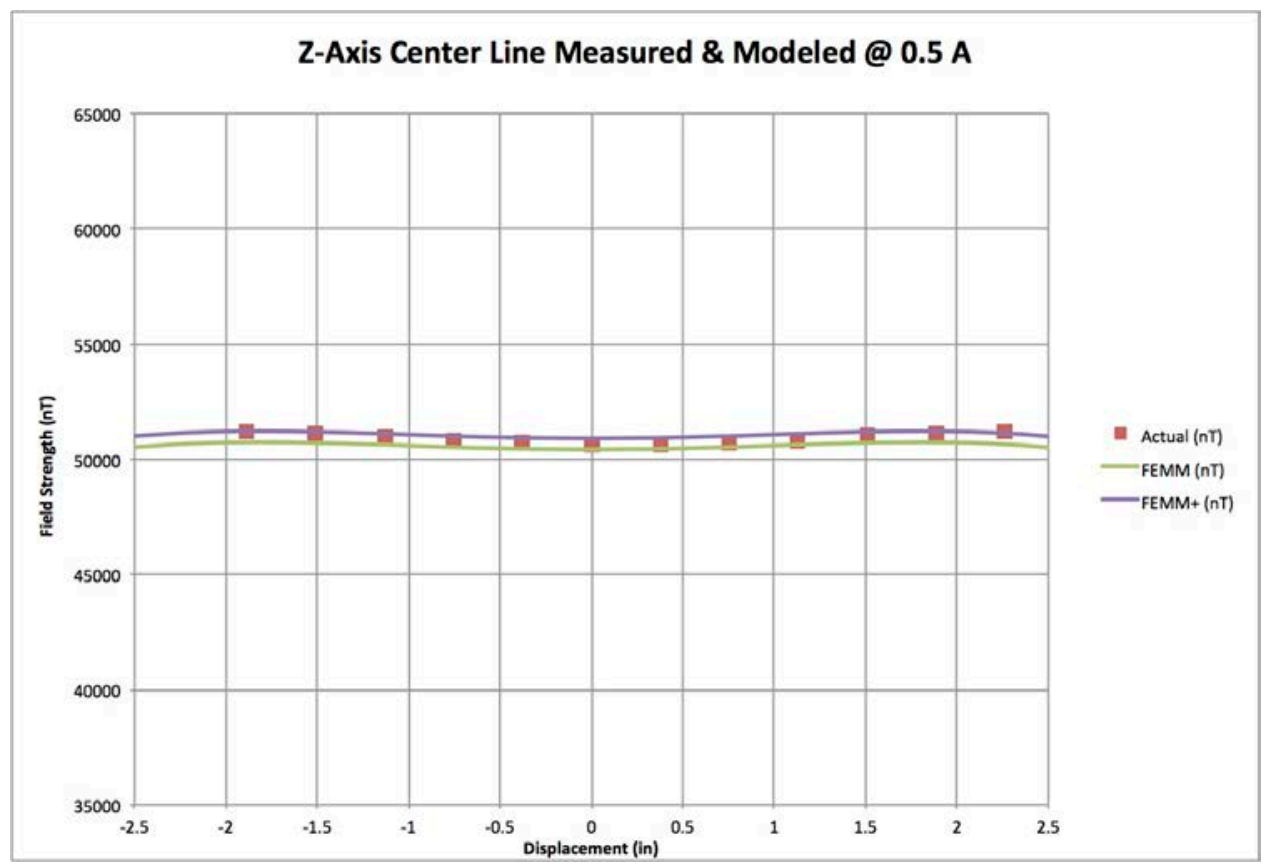

Figure 21: Z-axis Comparison of Measurements to Model

By using the in-axis profile measured from each coil and comparing it to the in-axis profile generated by the finite element model, confidence is gained that the model is correct and can be used to determine offsets for other points in the internal volume. Even with the non-uniformity created by inaccuracy in the as-built coil spacing, the field should be symmetrical around the coil axis due to the symmetrical nature of Helmholtz coils, and easily characterized by the model.

In practice, the end goal is to understand the actual field values at the location of each magnetometer at the center of the 6 exterior faces of a $10 \mathrm{~cm}$ cube, then compare those values with the values reported by the magnetometers. To get a calibrated actual field value from the Helmholtz cage the vector magnetometer is placed in the center and each coil is energized with a known current. To determine the actual field at each of the magnetometer locations, an offset is required with respect to that center location, and that offset is determined by looking at the in-axis and cross-axis profiles generated by the 
finite element model. The in-axis profiles are shown above as part of the comparisons in Figure 19, Figure 20, and Figure 21. The cross-axis profiles are shown below in Figure 22, Figure 23, and Figure 24.

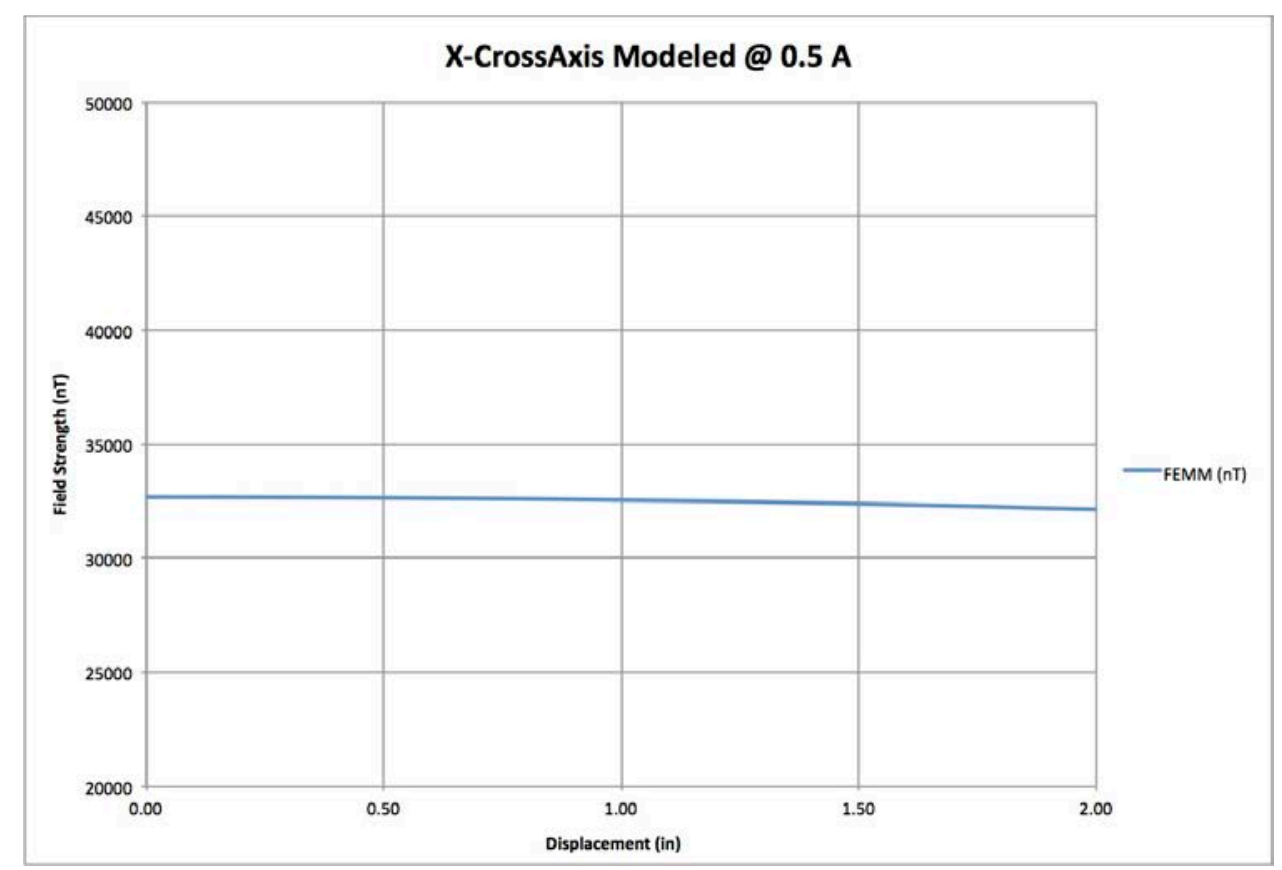

Figure 22: X-Axis Cross-Axis Profile from Model

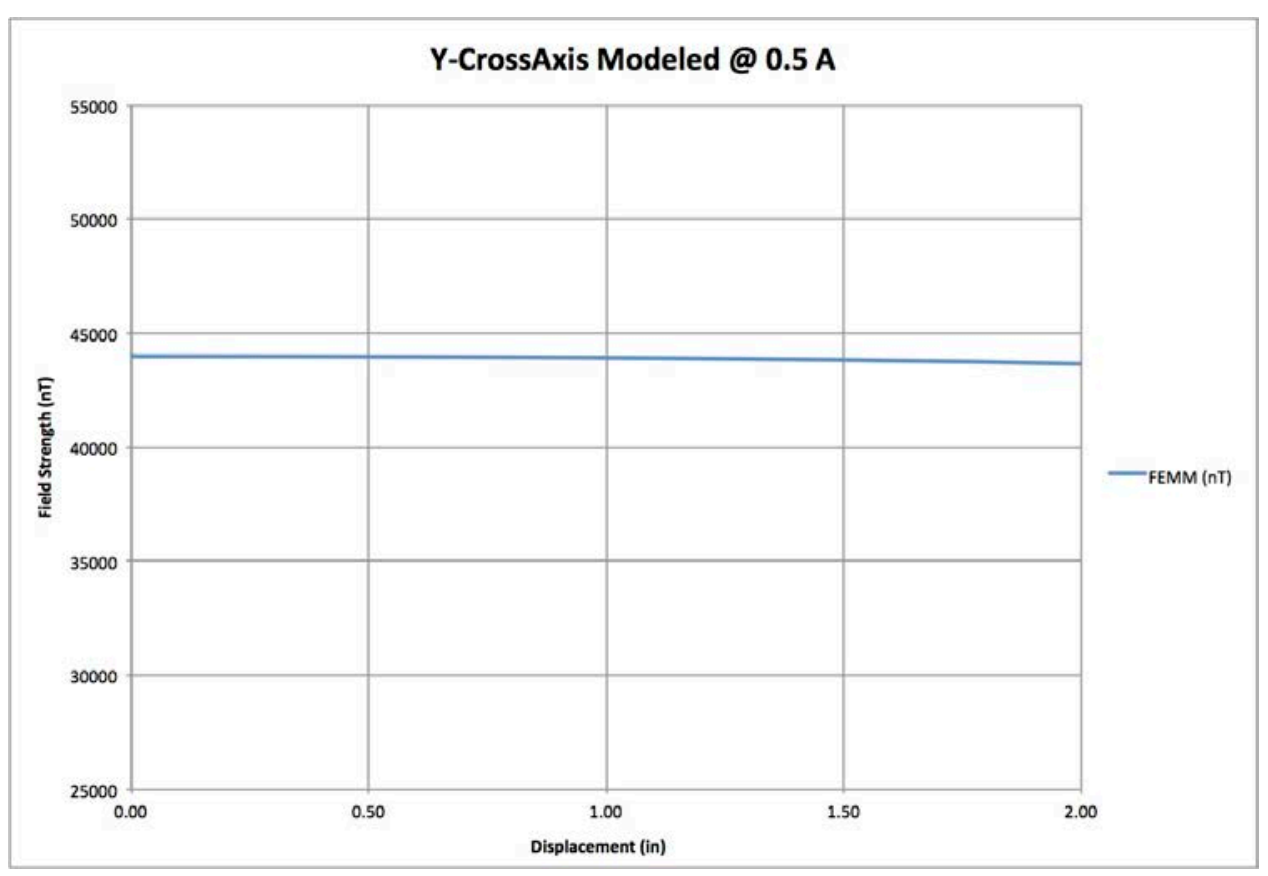

Figure 23: Y-Axis Cross-Axis Profile from Model 


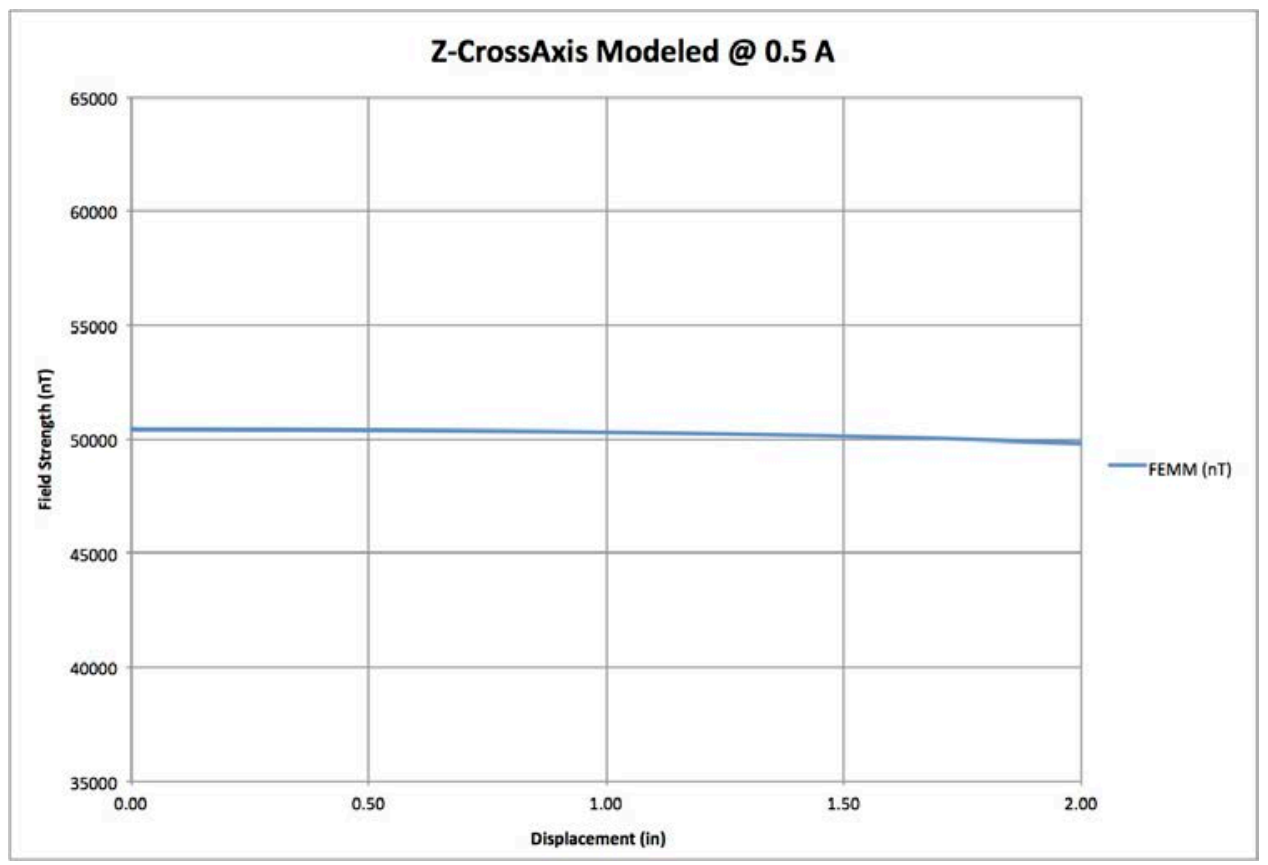

Figure 24: Z-Axis Cross-Axis Profile from Model

By using the previous profiles, the offsets between the center point and the magnetometer locations can be determined, both in-axis and cross-axis. The offsets are summarized below in Table 1.

Table 1: Helmholtz Field Offsets at Sensor Locations

$\begin{array}{lrrr}\text { Sensor Location } & \text { X-offset (nT) } & \text { Y-offset (nT) } & \text { Z-offset (nT) } \\ +/-X & 876 & -331 & -605 \\ +/-Y & -539 & 207 & -605 \\ \text { +/-Z } & -539 & -331 & 300\end{array}$




\section{CALIBRATION METHOD FOR THE MAGNETIC SENSOR}

Initial Testing of the HMC5883L Magnetometer

The first step in learning about how the HMC5883L magnetometer works is to test one in its most isolated form. A development board is available, shown in Figure 25, that enables convenient connection to the power and data lines with minimal nearby support electronics, and a small printed circuit board makes for convenient mounting. The data lines use standard $\mathrm{i}^{2} \mathrm{c}$ signals. Using an Arduino kit coded with a simple request and forward program, the magnetometer can be turned on and read over a virtual serial port.

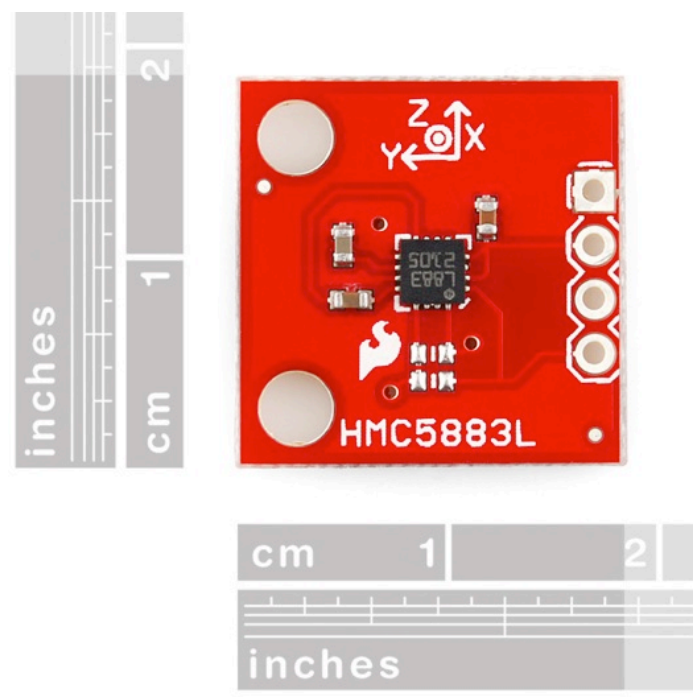

Figure 25: HMC5883L Development Board

The Arduino places the magnetometer into continuous-read mode, sets the scale to the default $+/-1.3$ Gauss (130 uT), and requests and collects the data. It returns the raw magnetometer values, the values corrected for the scale mode, and an estimated compass heading in radians and degrees as shown in Figure 26. 


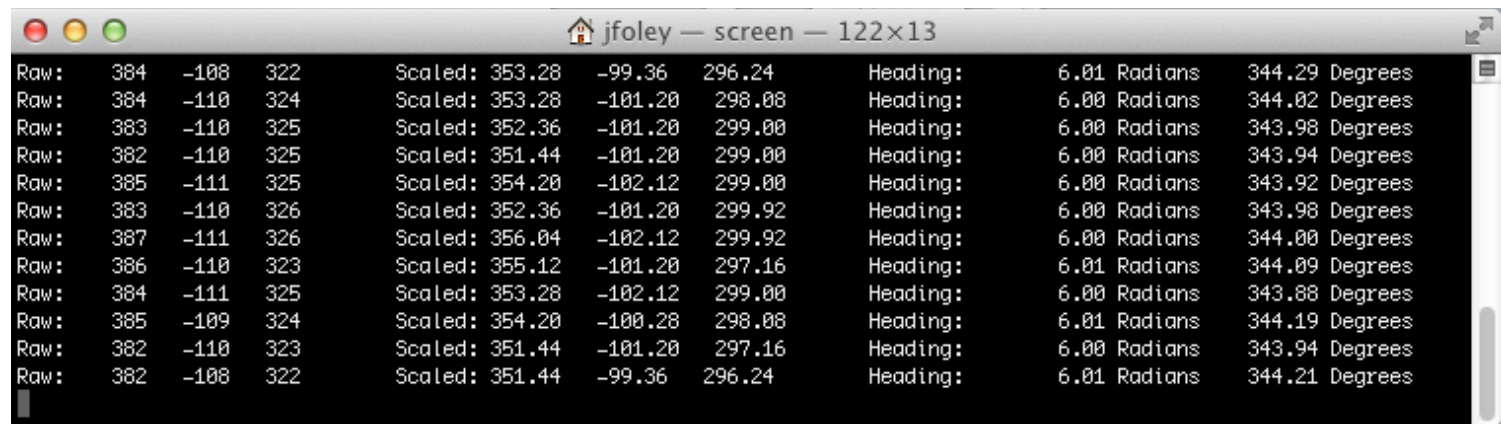

Figure 26: Output from HMC5883L through Arduino

For initial testing of the HMC5883L, the test setup is as follows:

- FVM-400 vector magnetometer on test stand for calibration

- RS-232 interface to computer for taking data

- HMC5883L development board on test stand

- Arduino USB interface to computer for taking data

- Test stand positioned in center of Helmholtz cage for each test

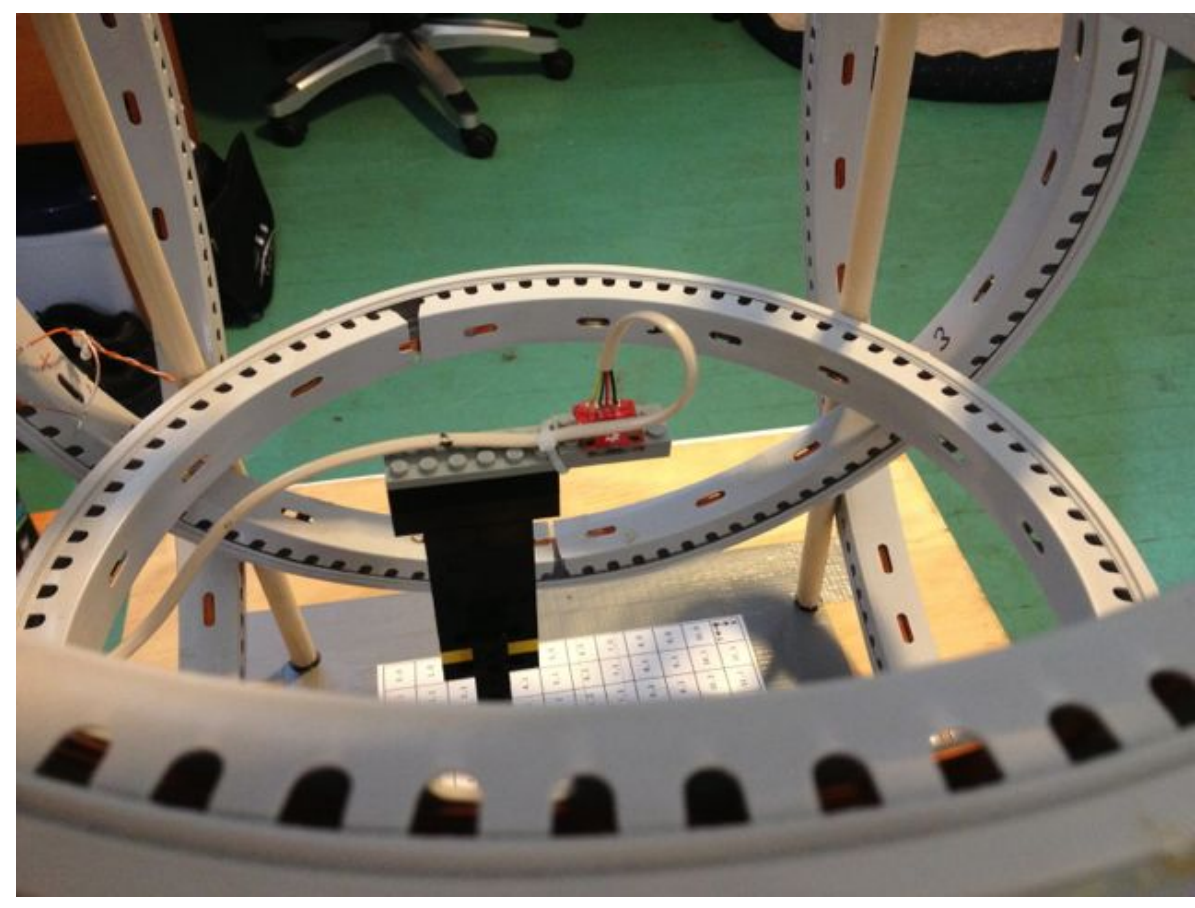

Figure 27: HMC5883L Development Board on Test Stand 
The tests are used to determine if calibration is required and to compare to the accuracy claimed on the HMC5883L datasheet of $1-2^{\circ}$ of compass heading [5]. To test the compass heading accuracy in all three dimensions, a magnetic field rotation is induced around the $\mathrm{Z}$-axis, testing the $\mathrm{X}$ and $\mathrm{Y}$ sensors, and around the $\mathrm{X}$-axis, testing the $\mathrm{Y}$ and $\mathrm{Z}$ sensors. The rotations are created by supplying a list of comma-separated vectors to the Helmholtz cage software. For these tests, the lists were generated in Excel. For example, to rotate about the $\mathrm{Z}$ axis, the following formulas were used, where $\mathrm{B}_{\mathrm{mag}}$ is the full field magnitude, $\mathrm{N}$ is the sample number and $\mathrm{T}$ is the number of samples in a period.

$$
\begin{gathered}
B_{x}=B_{m a g} \times \sin \left(\frac{\pi N}{T}\right) \\
B_{y}=B_{m a g} \times \cos \left(\frac{\pi N}{T}\right) \\
B_{z}=0
\end{gathered}
$$

Due to local magnetic field strength and limitations of the power supply, the maximum field initially tested was a $30000 \mathrm{nT}$ vector rotated about the Z-axis. The resulting data is compared to the actual field data to determine whether calibration is necessary and what accuracy is achieved. Prior to running the test, the FVM-400 vector magnetometer is used with the calibration procedures to prepare the Helmholtz cage. The values read and recorded from the HMC5883L are the scaled values as shown in Figure 26. Data from the first test are shown in Figure 28 and Figure 29. 


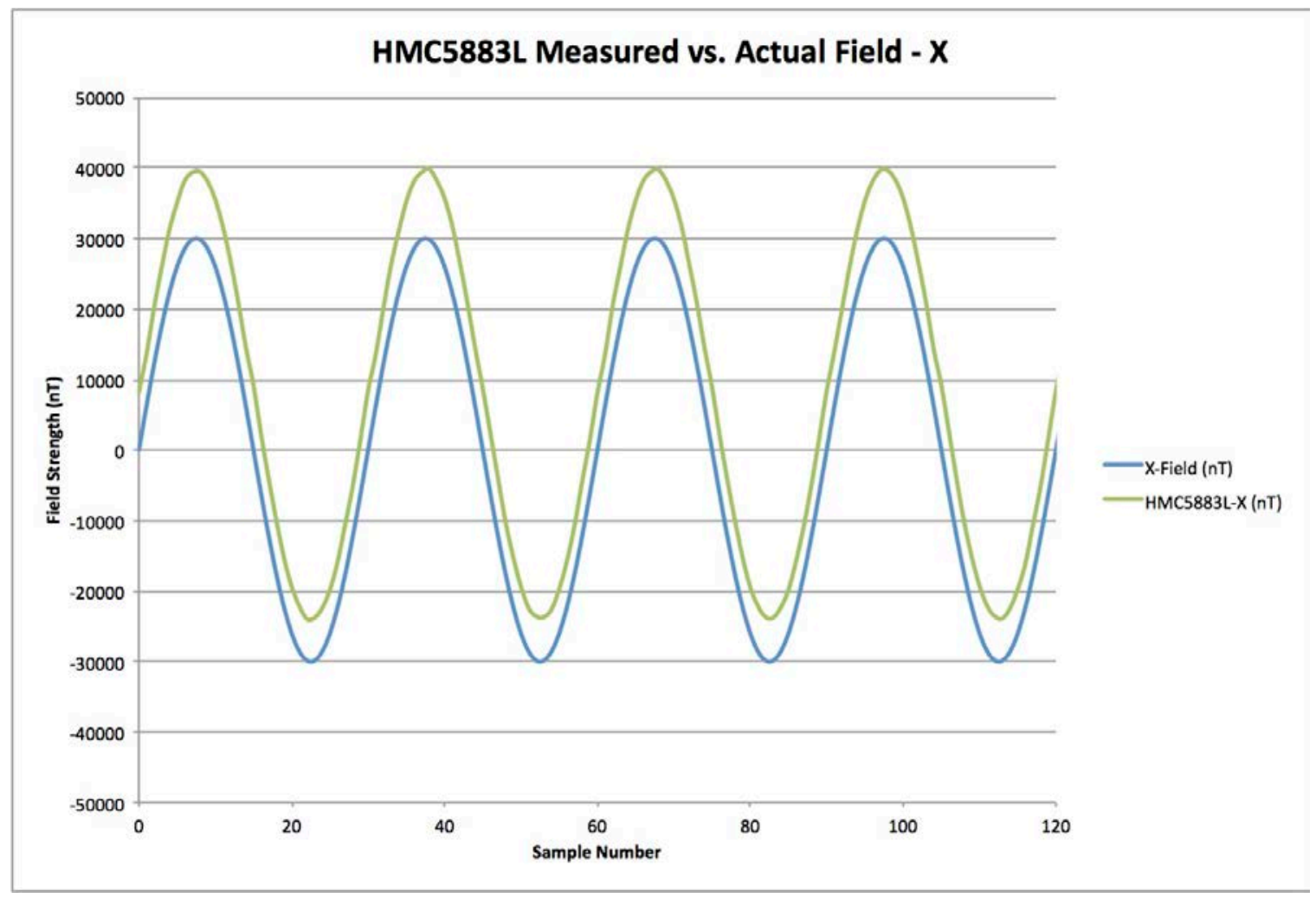

Figure 28: First Test of HMC5883L X-Data

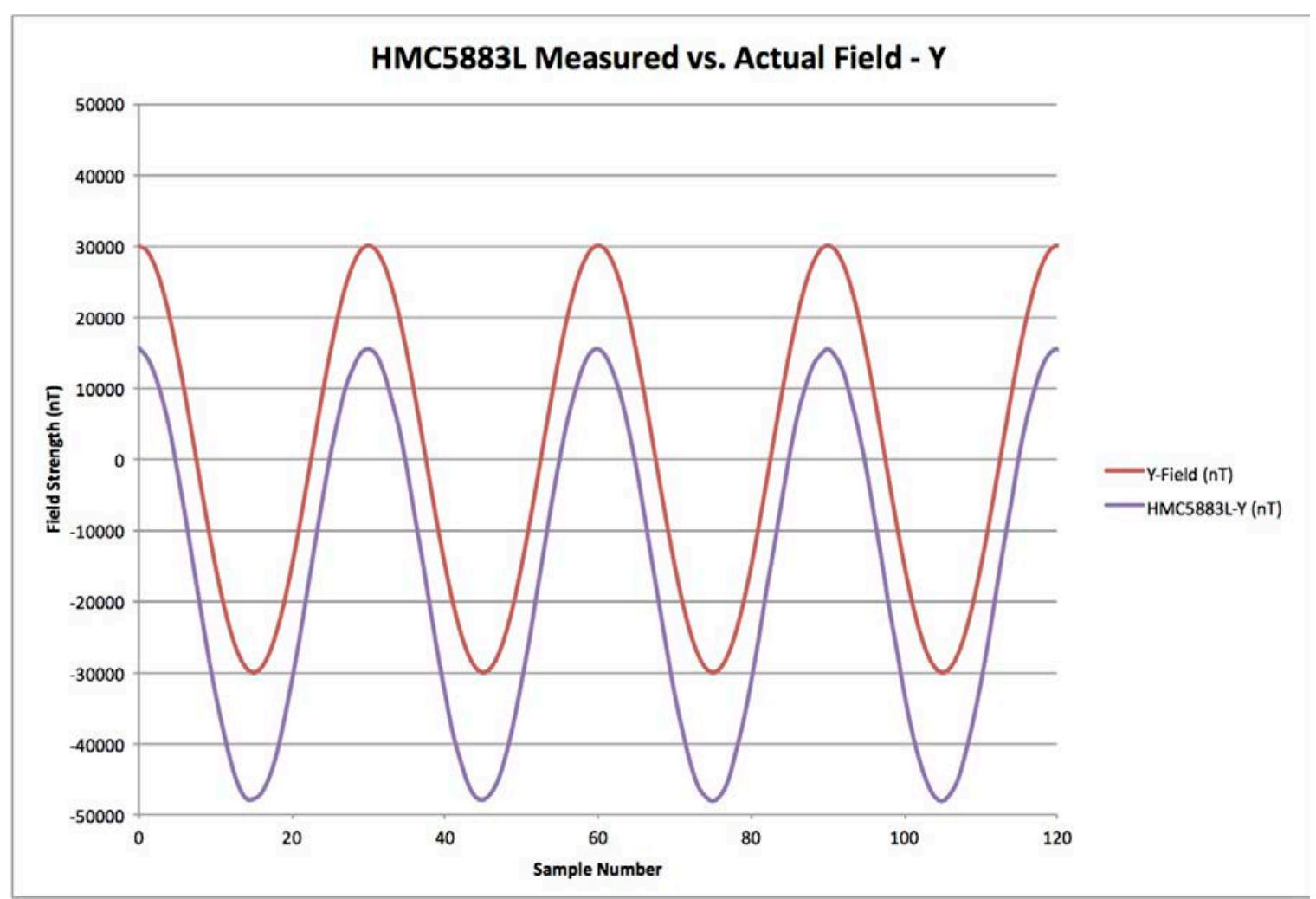

Figure 29:First Test of HMC5883L Y-Data 
As seen from the data in the previous plots, the field readings are considerably inaccurate without any calibration. By plotting the reported values from the HMC5883L against the actual values induced by the Helmholtz cage, it can be shown that the relationship between the two is linear.

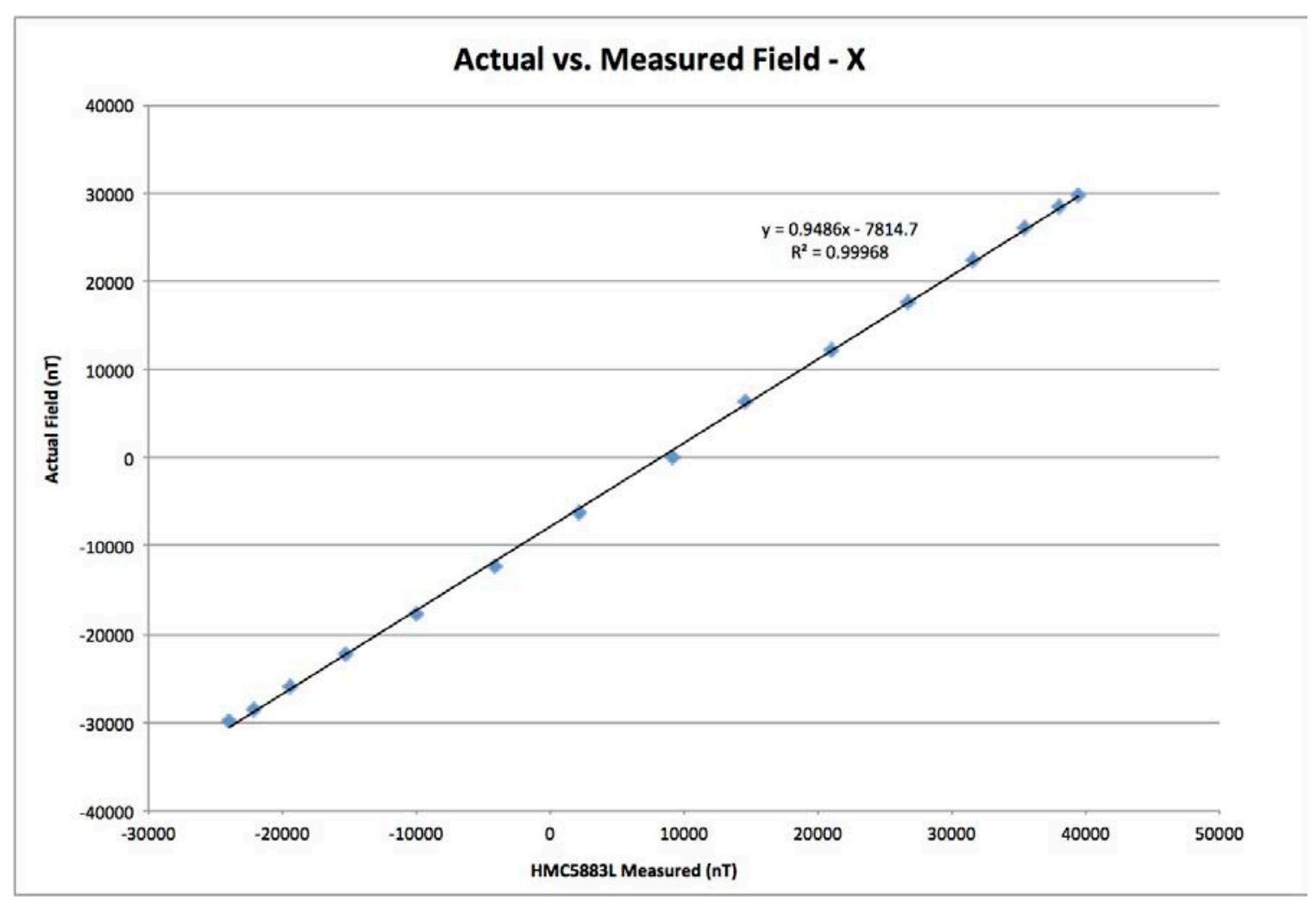

Figure 30: X Linear Calibration 


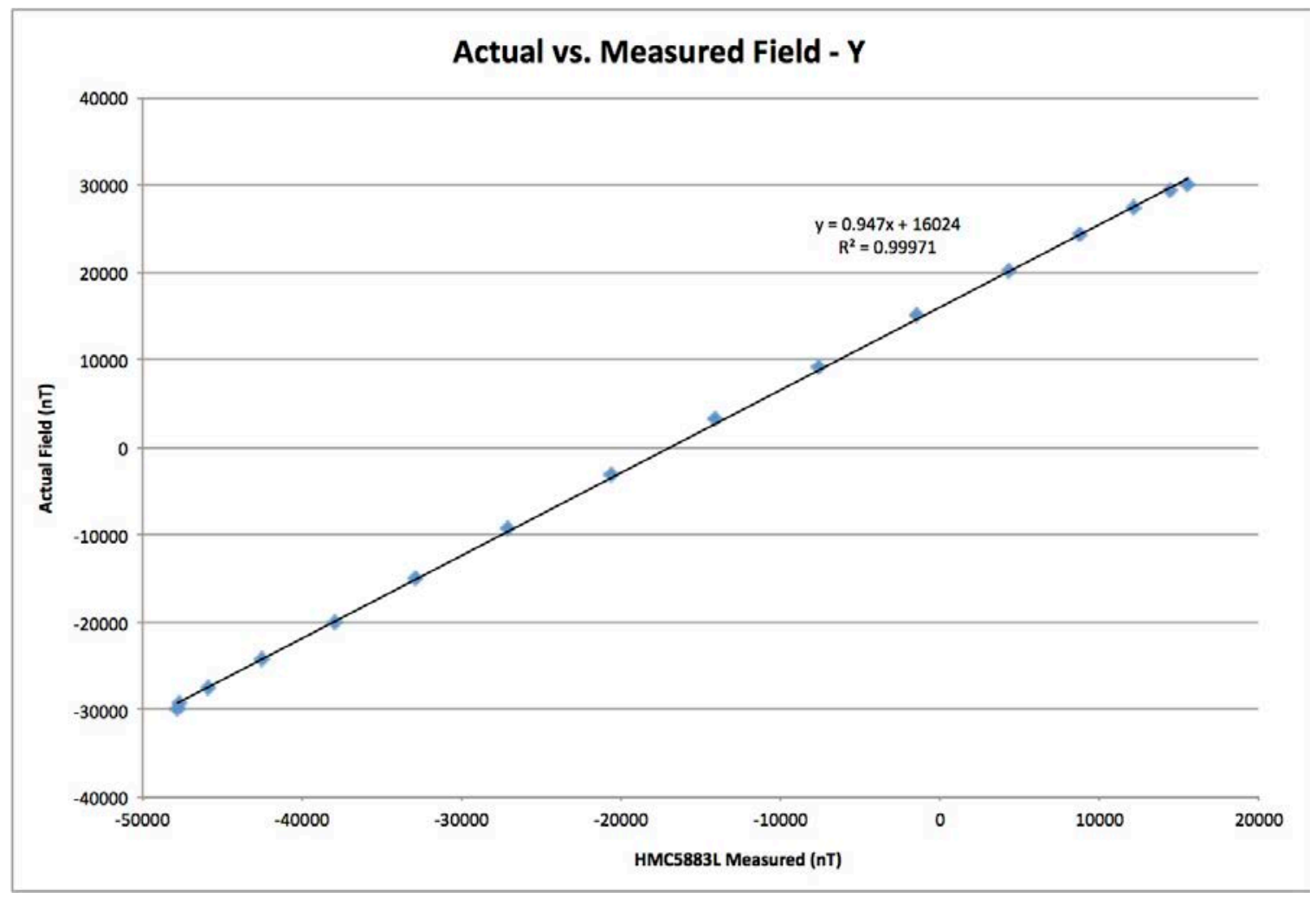

Figure 31: Y Linear Calibration

Using the linear trendline equations as shown in Figure 30 and Figure 31, the HMC5883L data can be calibrated and again plotted alongside the actual field values to compare uniformity. 


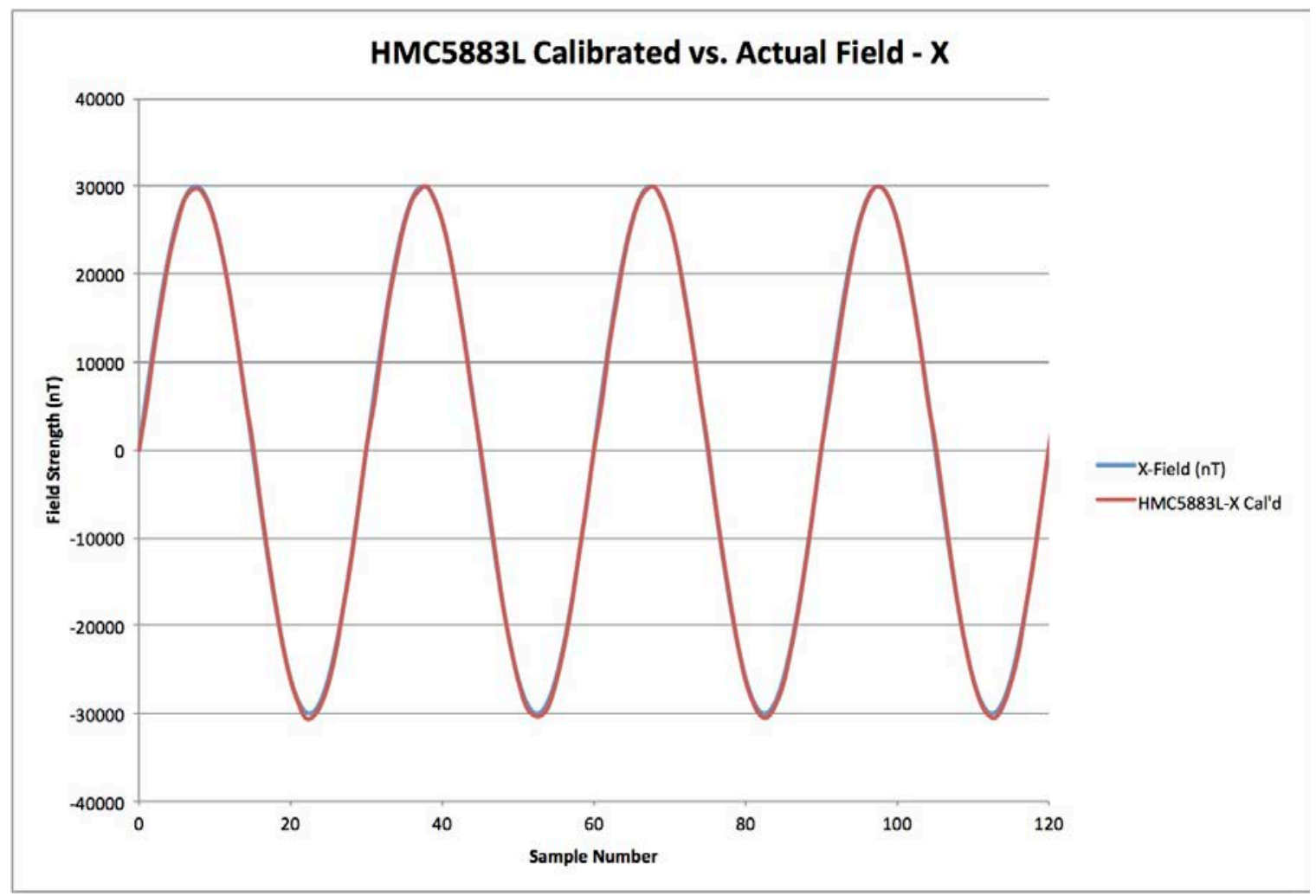

Figure 32: Calibrated X Data

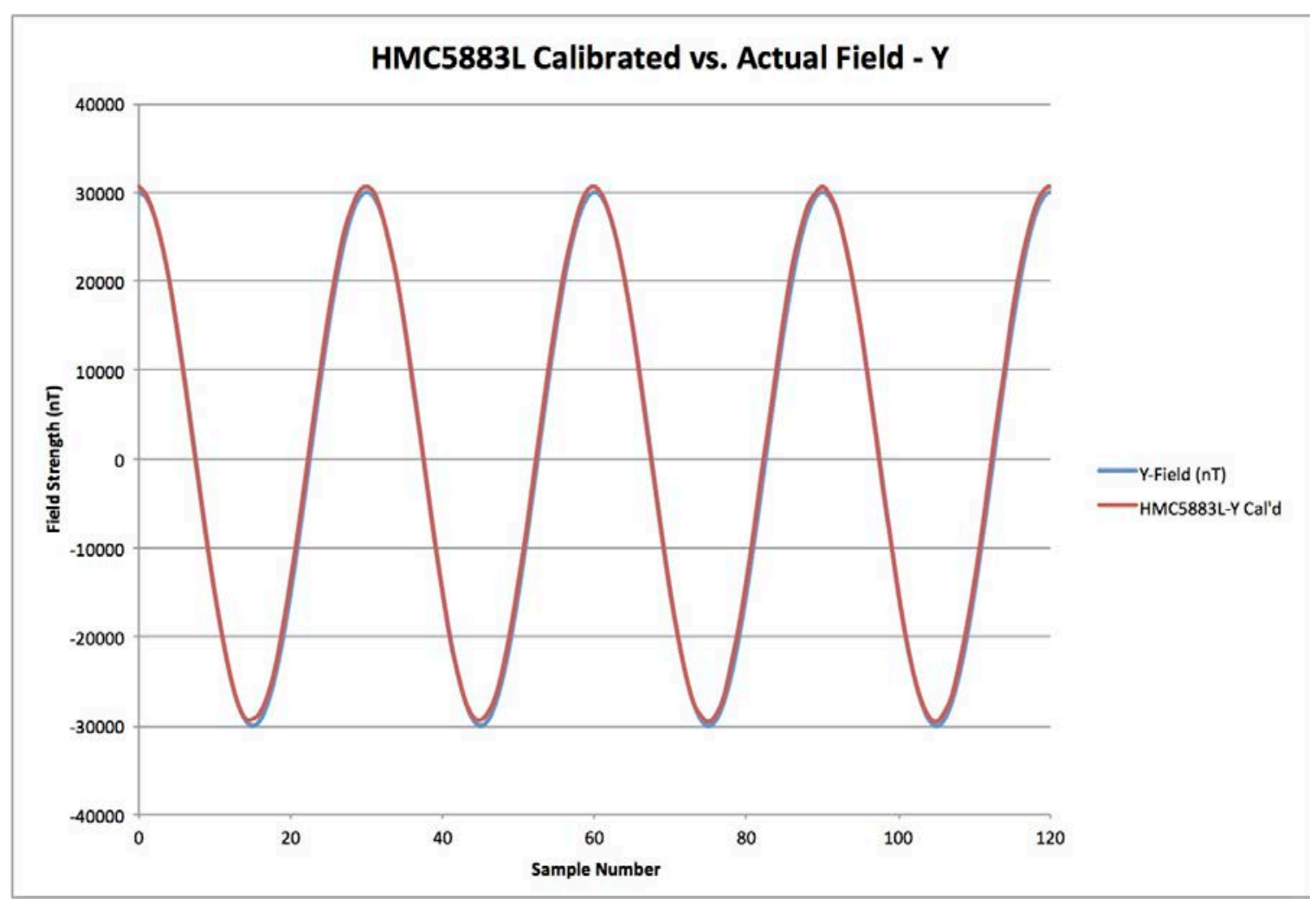

Figure 33: Calibrated Y Data 
To compare the results from an angle error perspective, the error is first represented as a difference, in magnetic units, between the actual magnetic field strength inside the Helmholtz cage and the field strength reported by the HMC5883L. Normally the compass-heading angle would be calculated by taking the inverse tangent of the $\mathrm{X}$ over $\mathrm{Y}$ components. The worst-case angle error as caused by a magnitude error in, for example, the $\mathrm{X}$-axis reading would result when the $\mathrm{X}$-axis is at a minimum state and the $\mathrm{Y}$-axis is at a maximum state, which would be roughly equal to the overall field magnitude. Therefore, the worst-case angle error resulting from X-axis field error can then be represented by taking the inverse tangent of the $\mathrm{X}$-axis error over the overall field magnitude. In this initial test, the overall field magnitude at any given time is $30000 \mathrm{nT}$.

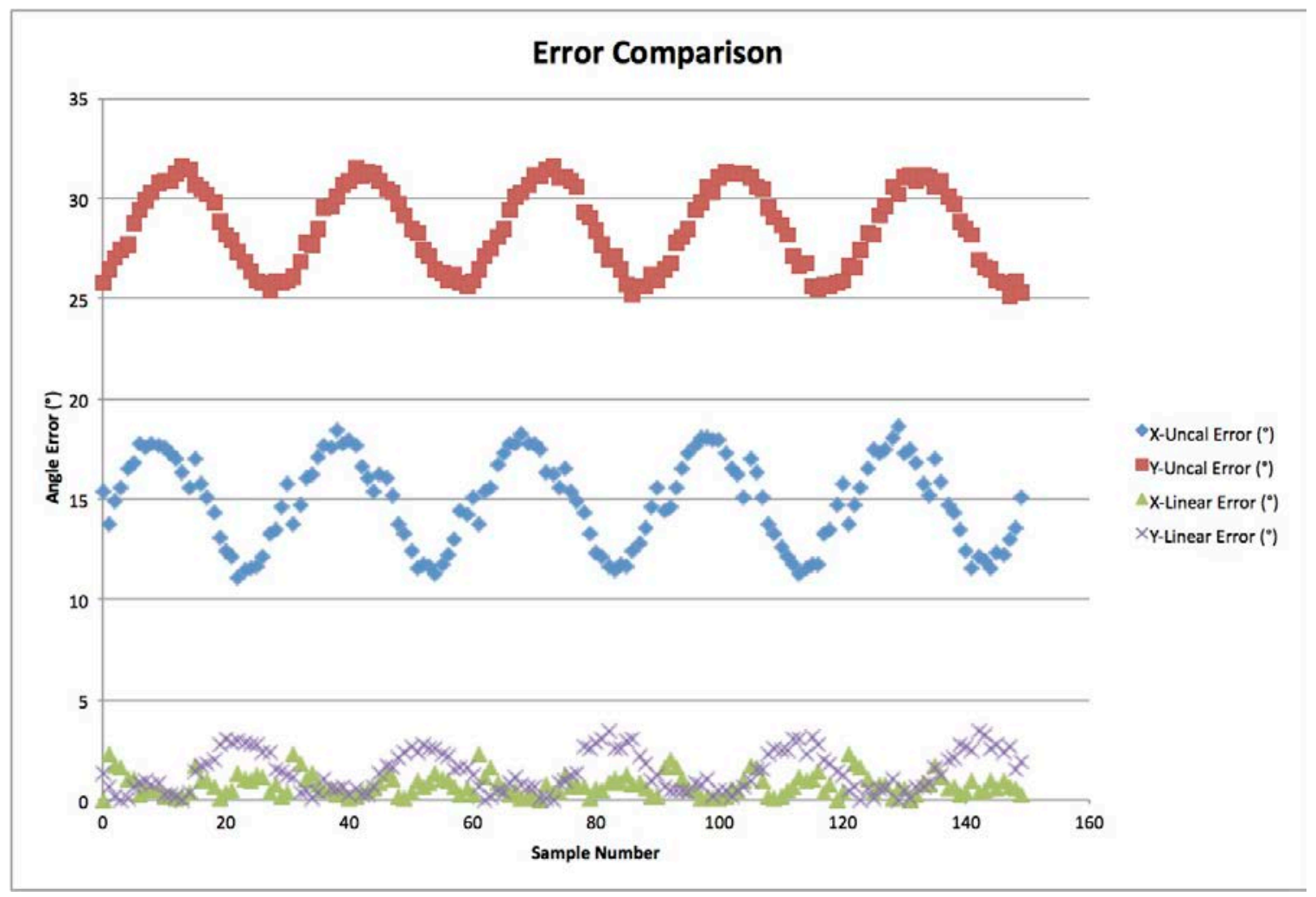

Figure 34: Comparison of Angle Error 
A comparison of the angle error both before and after the linear trendline calibration is applied is shown above in Figure 34. To summarize the results, the average angle error in the $\mathrm{X}$ axis is improved from $14.9^{\circ}$ uncalibrated to $0.7^{\circ}$ calibrated. The average angle error in the $\mathrm{Y}$-axis is improved from $28.9^{\circ}$ uncalibrated to $1.4^{\circ}$ calibrated. It is worth noting this first test was performed informally in a home office, with only minor care taken to correctly align the magnetometer and minimize nearby magnetic interference, therefore some improvement is expected when testing within a more controlled environment. 


\section{Full Field Strength Testing}

With the test setup moved to a more controlled laboratory environment, the initial testing could be expanded upon to include a full magnetic field strength of $50000 \mathrm{nT}$. Procedures are nearly identical to the previous testing with rotations about the $\mathrm{X}$ and $\mathrm{Z}$ axes to test all three sensor components. Extra care was also taken to ensure that magnetometer placement was precise and aligned correctly with the axes of the Helmholtz cage.

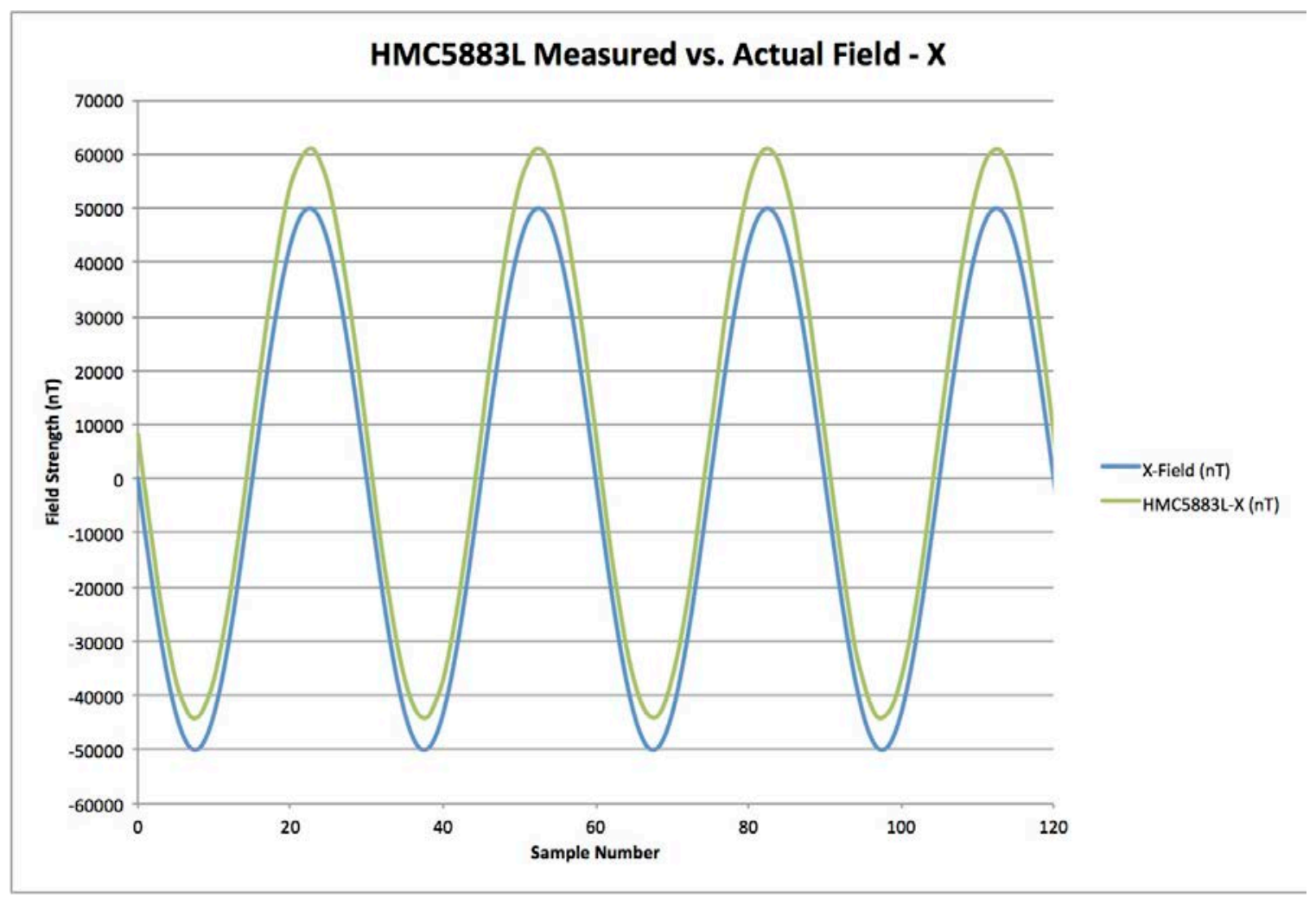

Figure 35: Full Strength Test Raw X Data 


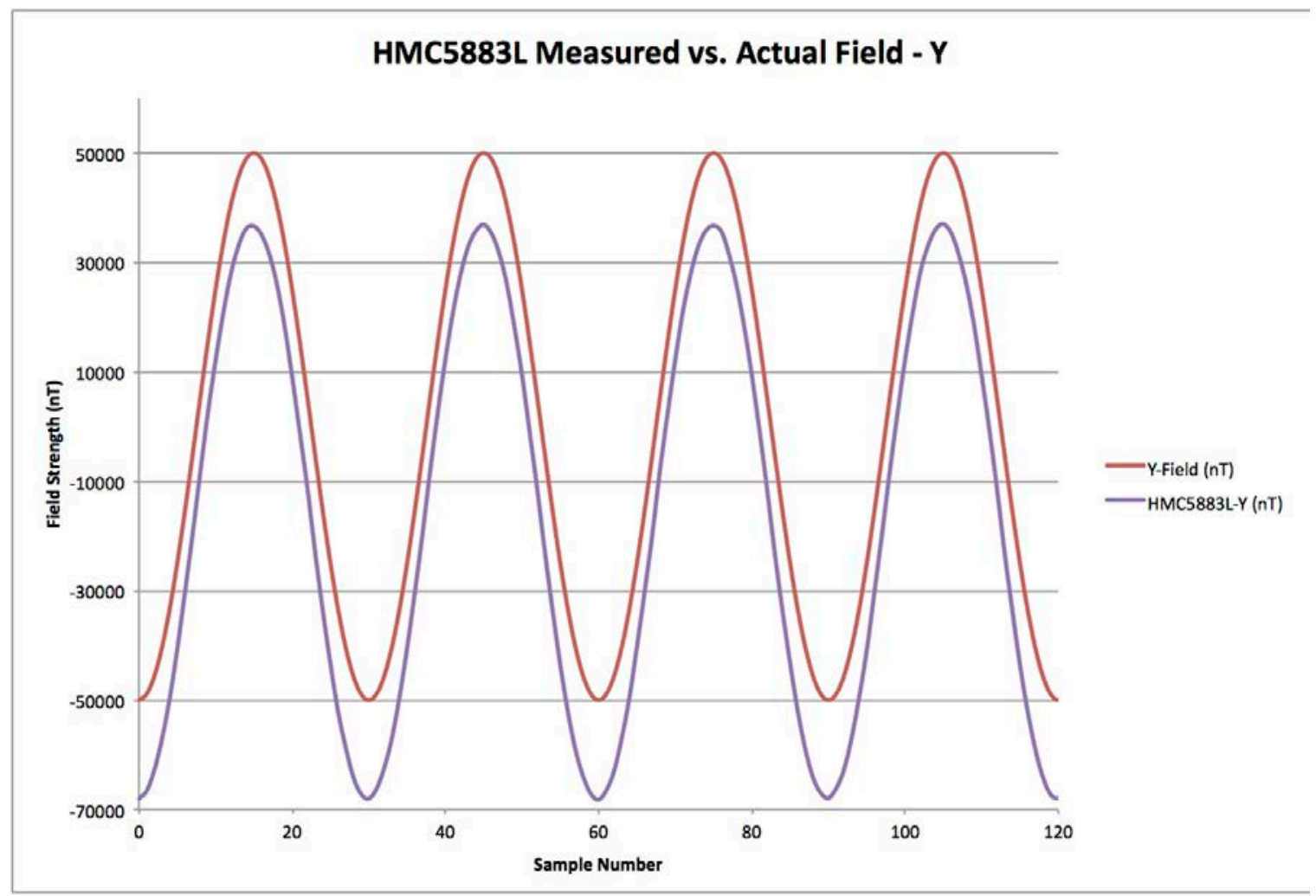

Figure 36: Full Strength Test Raw Y Data

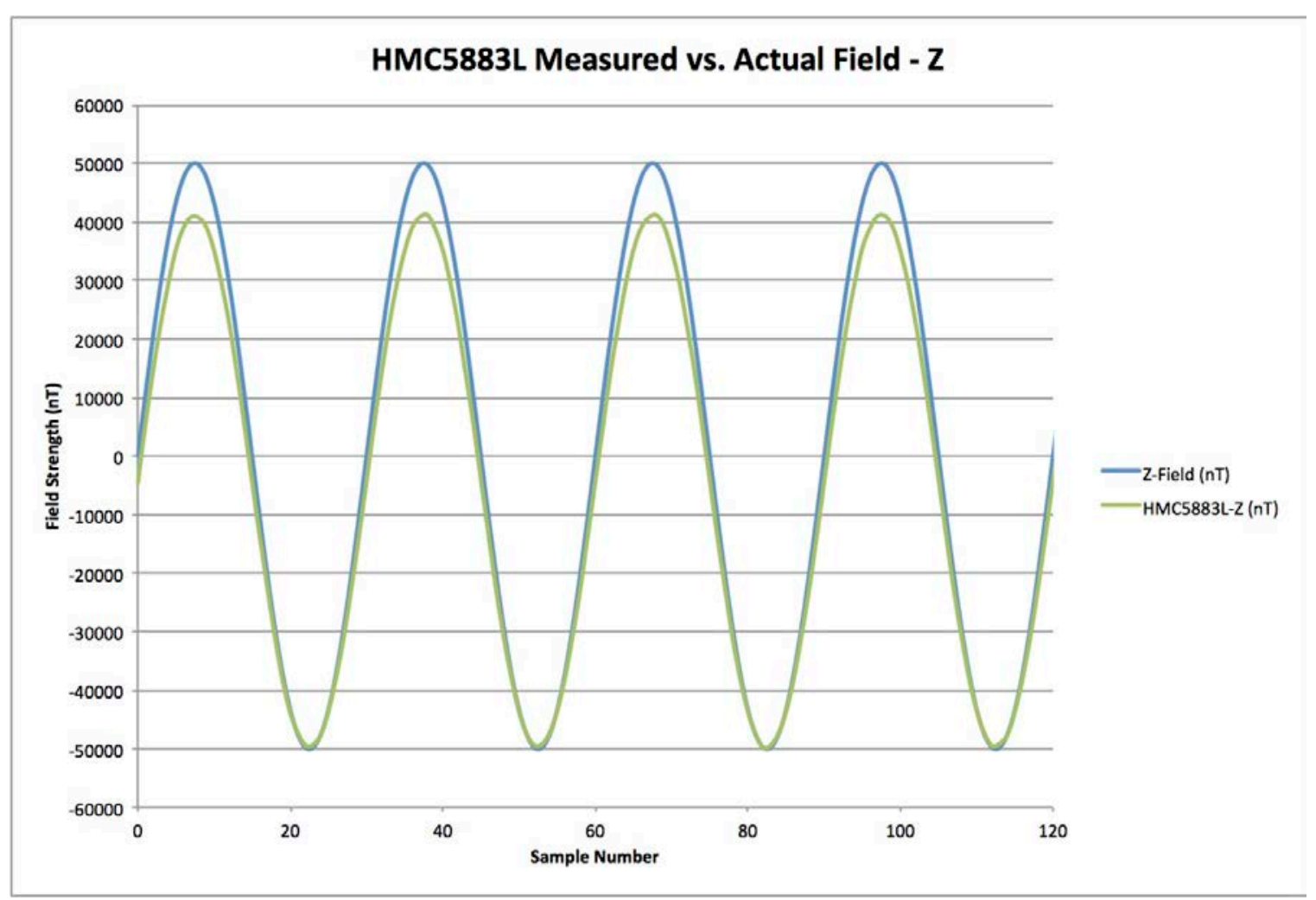

Figure 37: Full Strength Test Raw Z Data 


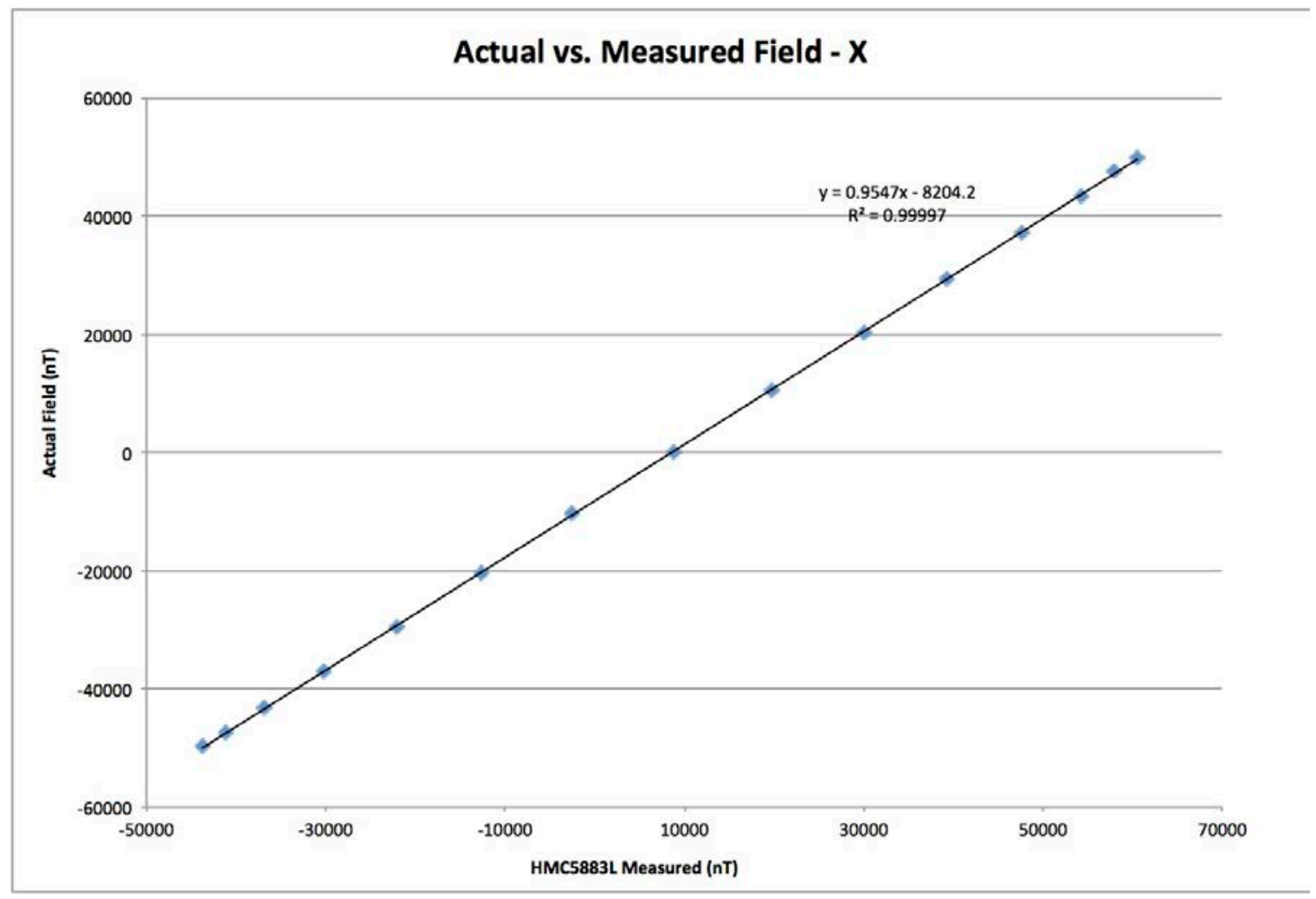

Figure 38: Full Strength Test X Linear Fit

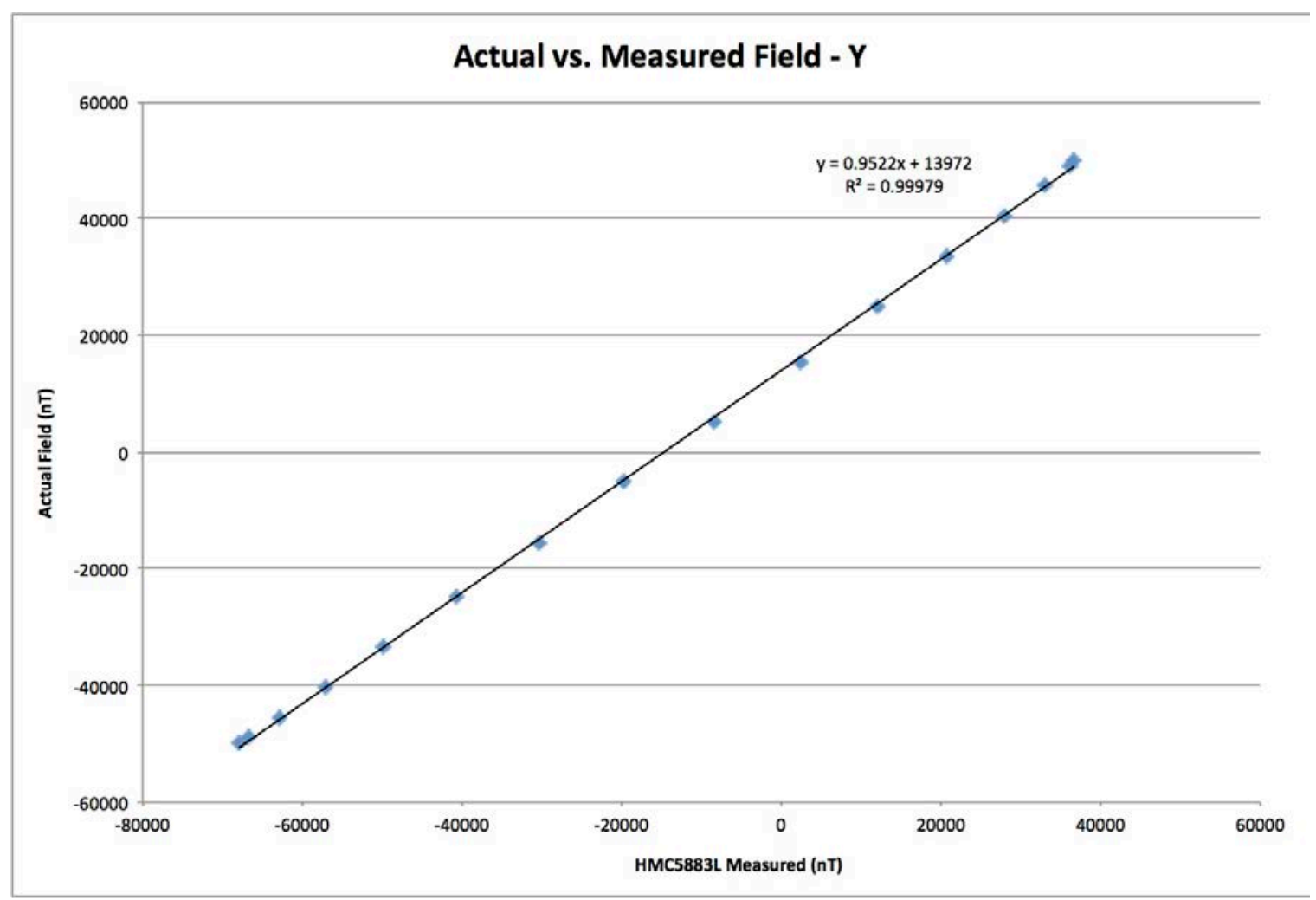

Figure 39: Full Strength Test Y Linear Fit 


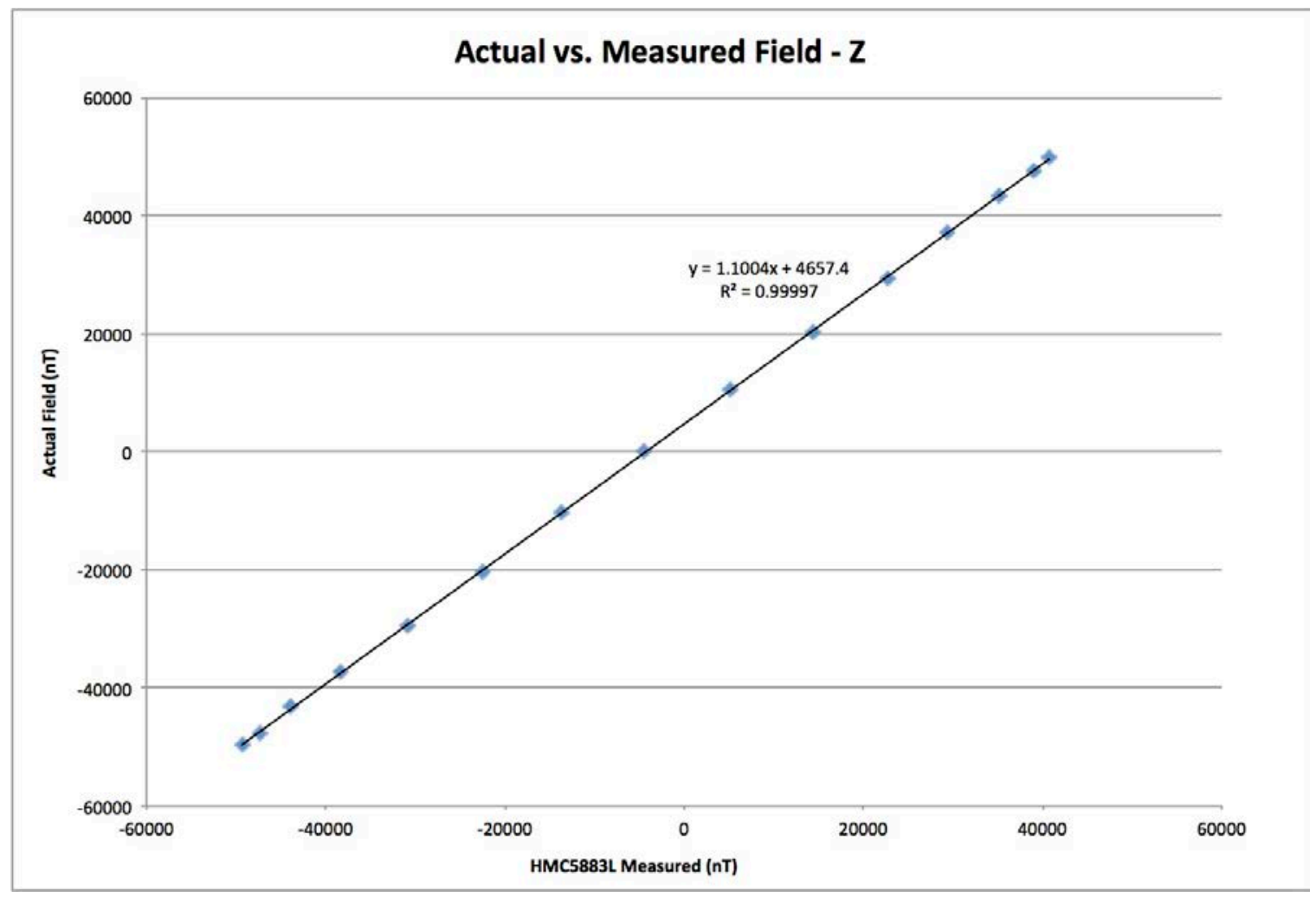

Figure 40: Full Strength Test Z Linear Fit

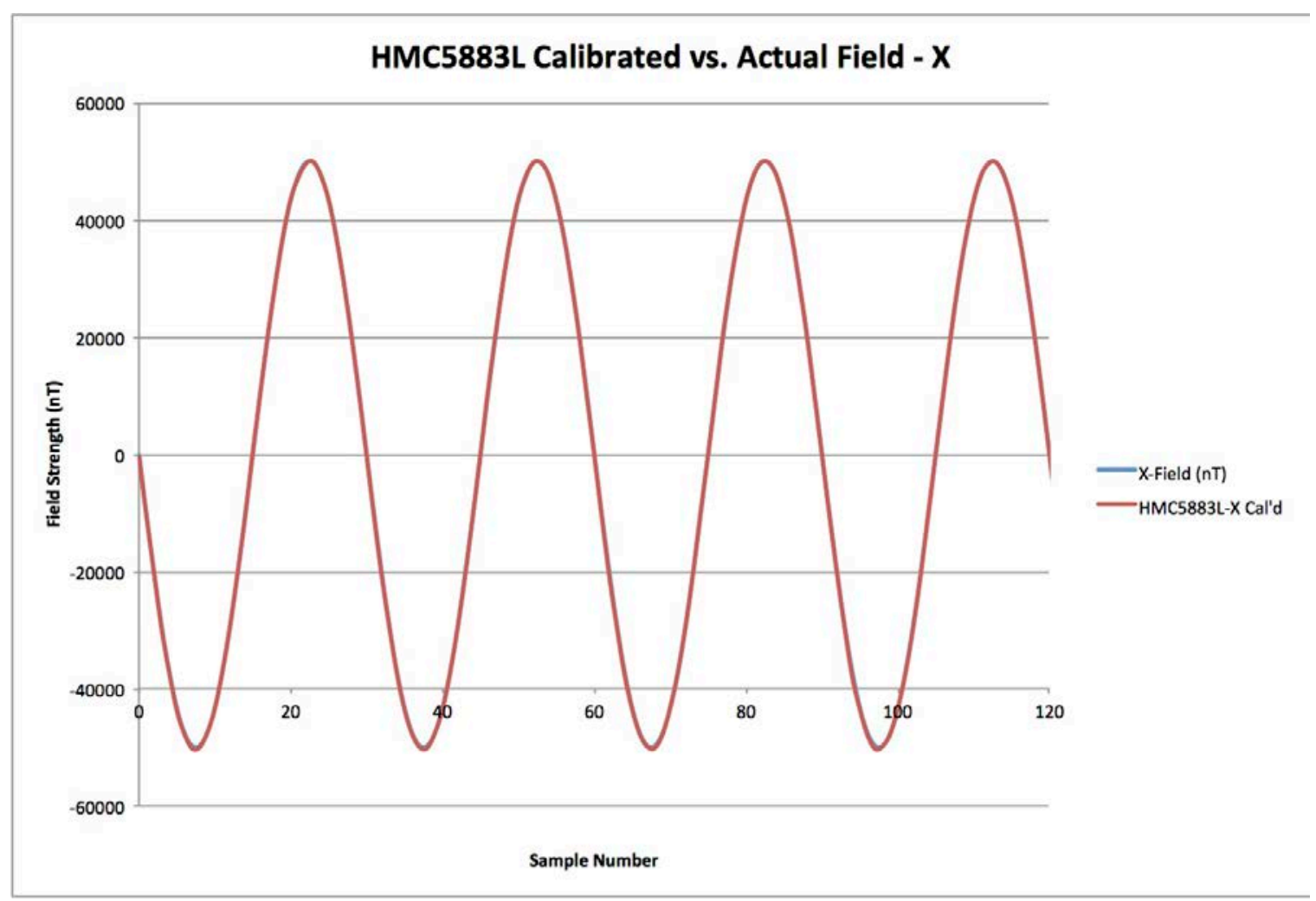

Figure 41: Full Strength Test X Calibrated 


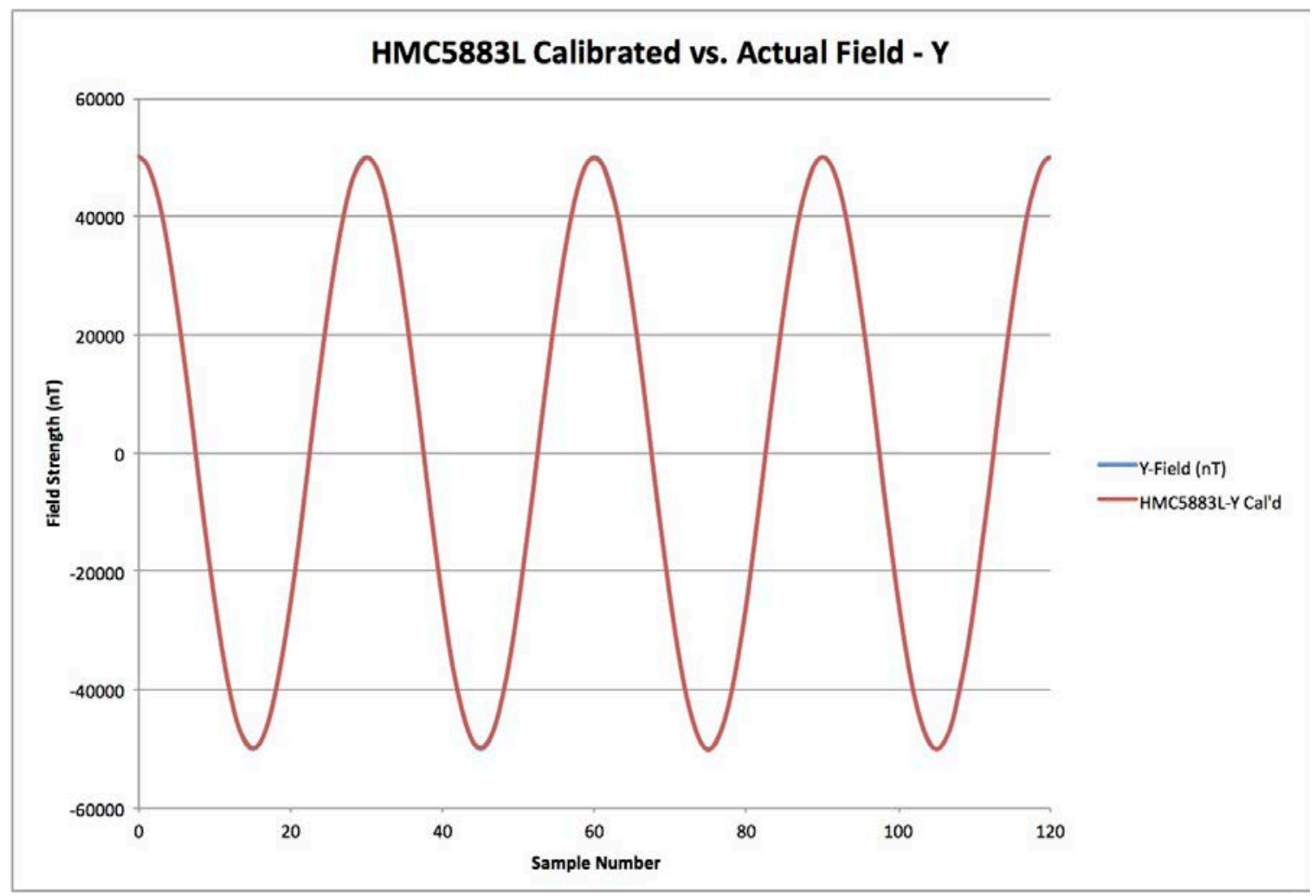

Figure 42: Full Strength Test Y Calibrated

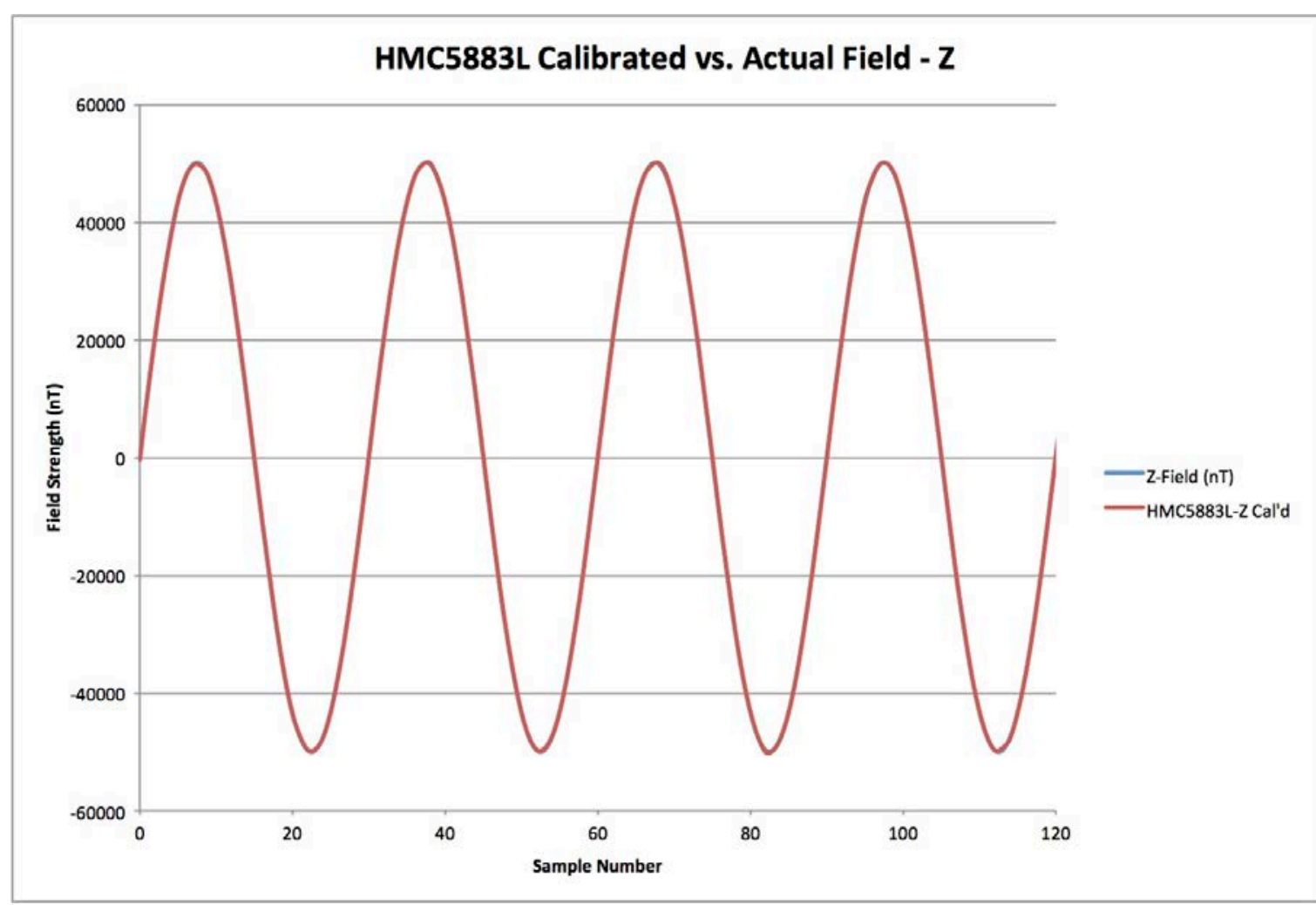

Figure 43: Full Strength Test $\mathrm{Z}$ Calibrated 


\section{MAGNETIC EFFECTS OF CUBESAT BUS}

The primary sources of error in the magnetometer readings are expected to be as a result of the magnetic fields introduced by satellite components in close proximity to the sensors. Passive effects could be as a result of high masses of metal, such as the satellite structure or batteries, and active effects could be as a result of electrical activity in the adjacent electrical circuits.

\section{Inactive Bus Effects}

Passive effects can be measured by running similar tests to the standalone HMC5883L tests, but attaching the HMC5883L to a flight-similar satellite bus. By performing the same calibration process with the satellite structure present, it can be tested whether the process will remain viable for the calibration of a fully built flight CubeSat. It also provides a way of testing the HMC5883L without running any of the necessary support electronics of the satellite bus, providing a separation between passive effects and active effects to be tested later.

The calibration tests in the Helmholtz cage were performed, as before, by simulating a rotation about the $\mathrm{X}$ and $\mathrm{Z}$ axes to exercise all three axes, with the HMC5883L development board attached to each of 5 of the 6 sides of the CubeSat structure. For brevity the full complement of calibration plots are omitted here, but the final results are presented below in Figure 44 and Figure 45 for comparison. An image of the test setup accompanying this data is shown in Figure 46. The average angle-error for the Y-axis data is $0.3^{\circ}$ and for the $\mathrm{Z}$-axis is $0.4^{\circ}$. 


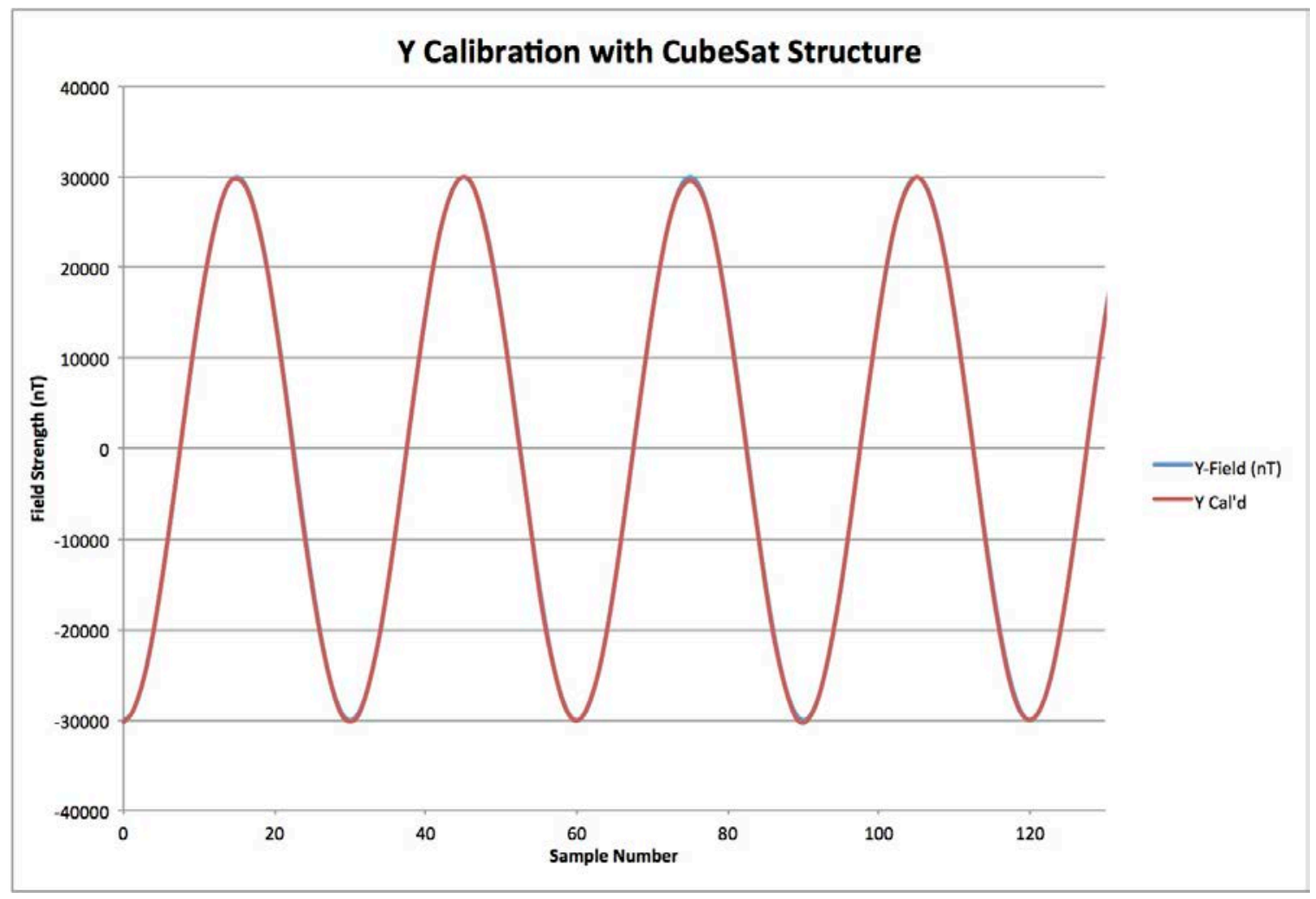

Figure 44: Y-Axis Data Attached to +Y Panel

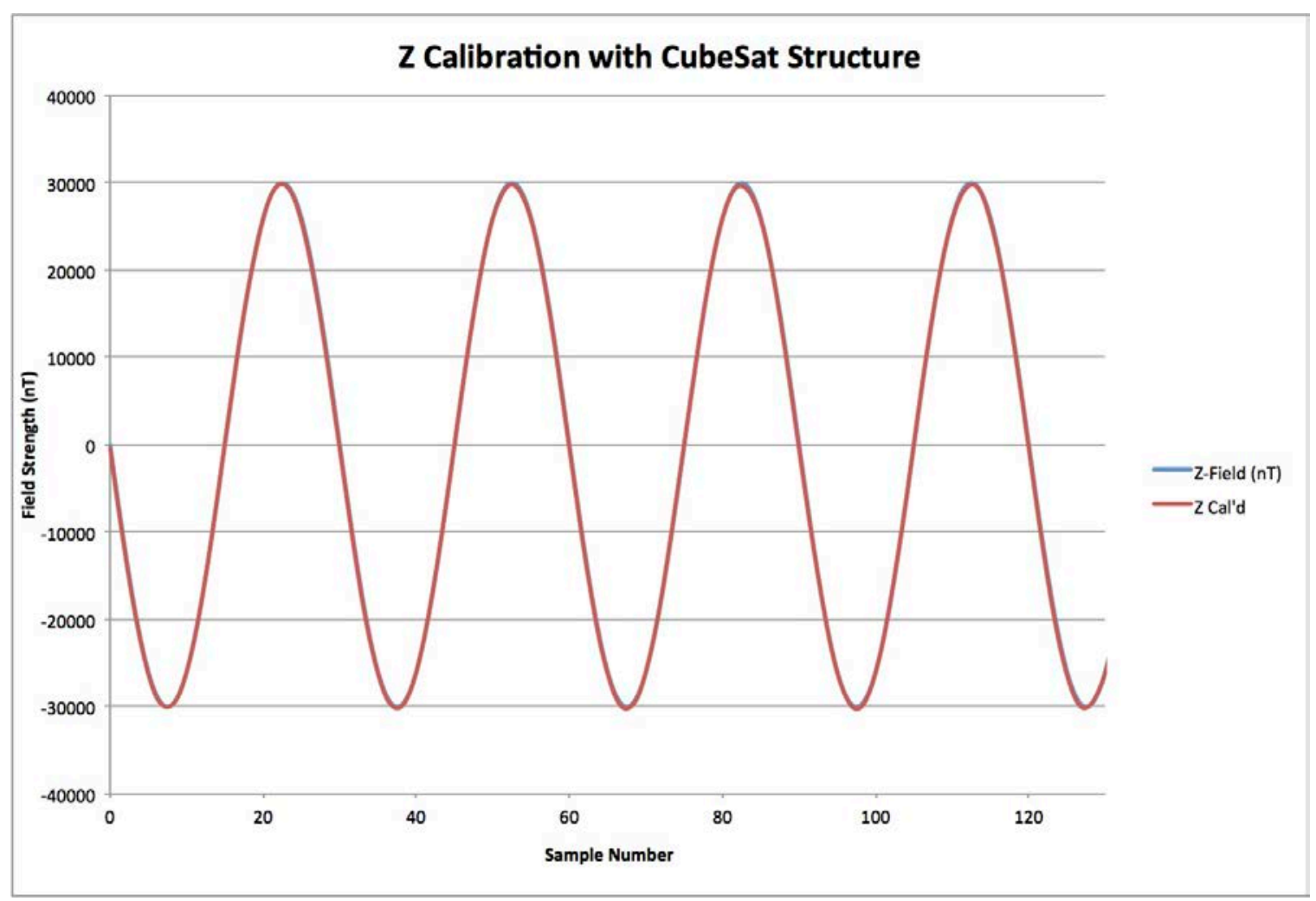

Figure 45: Z-Axis Data Attached to +Y Panel 


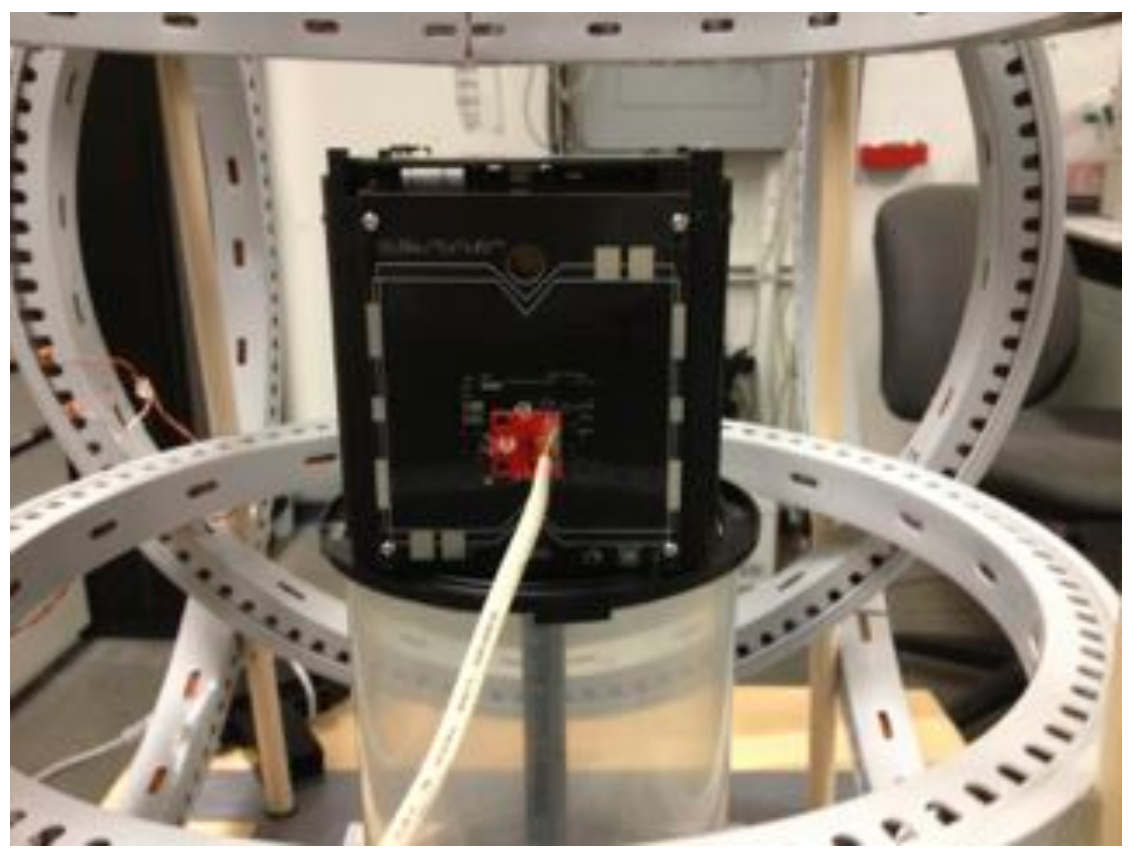

Figure 46: Inactive Bus Test Setup

Repeating the same calibration test with the HMC5883L development board attached to a flight-similar satellite bus indicates negligible effect on the calibration process. The materials used in the bus were an aluminum structure, non-functional system board and side panels without solar cells attached. 


\section{Active Bus Effects}

In order to remove the effects of active magnetic interference, the fields produced by the electronics must be measured in different modes of expected operation. The modes that cover most generic satellite operations are power-off, boot-up, idle, active CPU and RF

transmission. At the time of testing, however, next generation bus hardware was not available to directly read side panel data or activate RF transmission. Measurements could still be taken of the main system board using the FVM-400 vector magnetometer positioned at each side panel location that will contain an HMC5883L. By following the calibration procedures in the future, the effect of RF transmission can be characterized as well and factored into the concept of operations should the transmissions have a significant effect on magnetic field reading. While each of these modes of operation were of interest, it is expected that only the idle and active CPU modes would provide the most useful calibration offsets with the hardware available.

For measuring the effect of basic active electronics, the test setup is as follows:

- Next generation satellite bus on plastic test-stand

○ Aluminum structure

- System board powered by internal batteries

- Umbilical to computer for commanding activity

- FVM-400 Magnetometer on test-stand, connected to computer

The satellite bus is placed on the plastic satellite test-stand, and the FVM-400 magnetometer is positioned directly adjacent to it where the HMC5883L chip would normally be. The umbilical is connected between the satellite and the computer that is 
running terminal software. Also on the computer is the FVM-400 magnetometerrecording program. The computer is started and records magnetic field data every 100 milliseconds, then after a few seconds have passed to measure the power-off state, the RBF pin is pulled to activate the satellite. Upon power-up, the satellite goes through the boot-up process that can be tracked on the computer terminal. Once booted and logged in, it is left for a few seconds to measure the field in idle mode. Then a program is started on the satellite that induces high CPU activity. After a few seconds of measuring, the RBF pin is re-inserted and the satellite is powered off. This process was repeated for each of the 6 sides of the cube as shown below in Table 2 .

All data taken by the FVM-400 vector magnetometer is converted to the same coordinate system as the satellite structure as shown by the photos in Table 2. For completeness, a plot of vector magnitudes of the raw data is shown below in Figure 47. However, in order to more clearly compare the measurements at each side, in Figure 48 the magnitude data is normalized by subtracting the local magnetic field then lining up in the time domain by plotting an average of each mode being tested for each position. It is these averages that offer a calibration offset for each magnetometer location depending on what mode the satellite is in. The worst case is for the top panel, which is expected due to the close proximity to all the active electronics. More detailed results are presented in Table 3 which lists the resulting individual component offsets for each sensor location and Table 4 which provides estimated angle errors as a result. 
Table 2: Test Setups For Each Active Bus Measurement Location

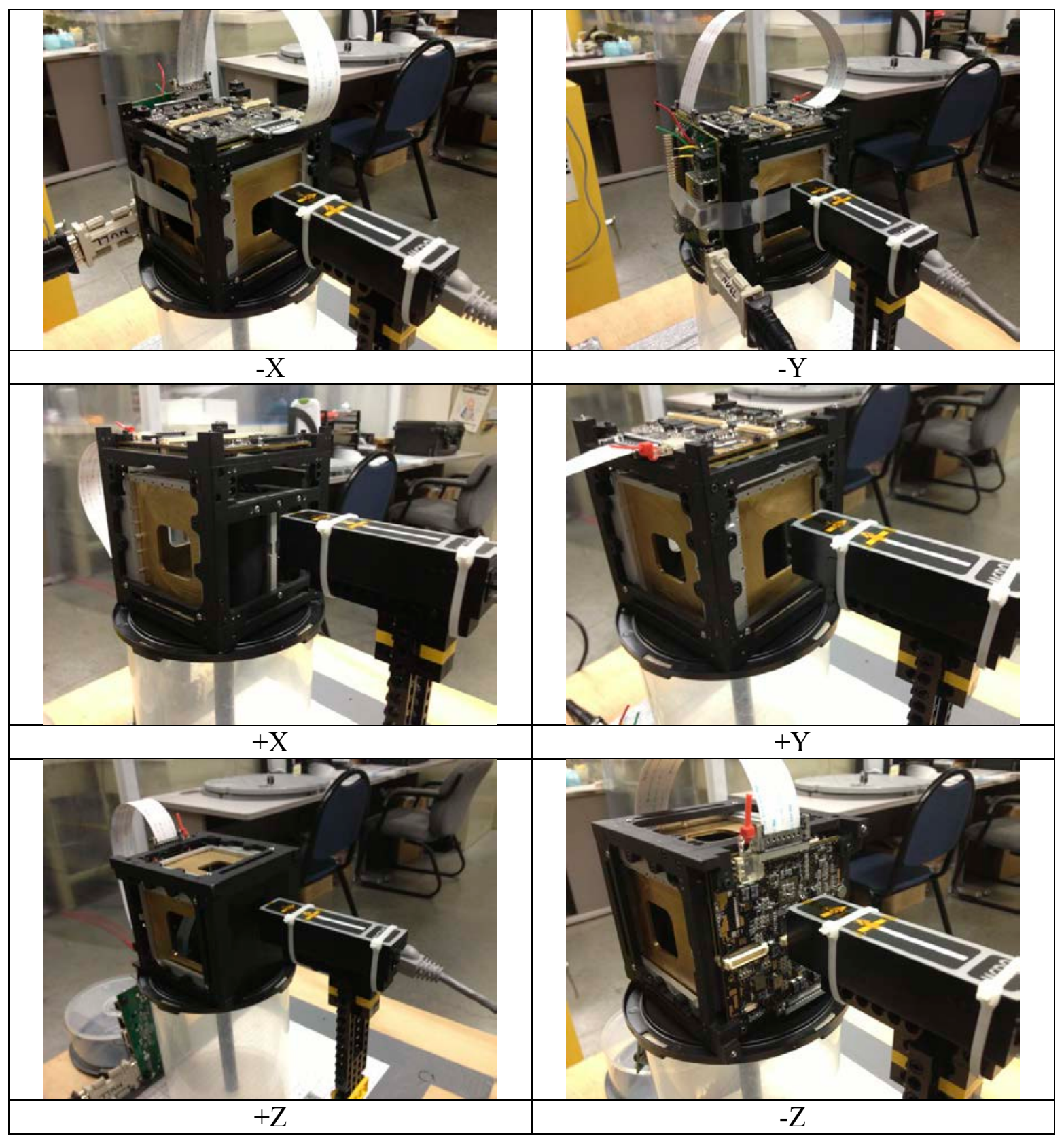




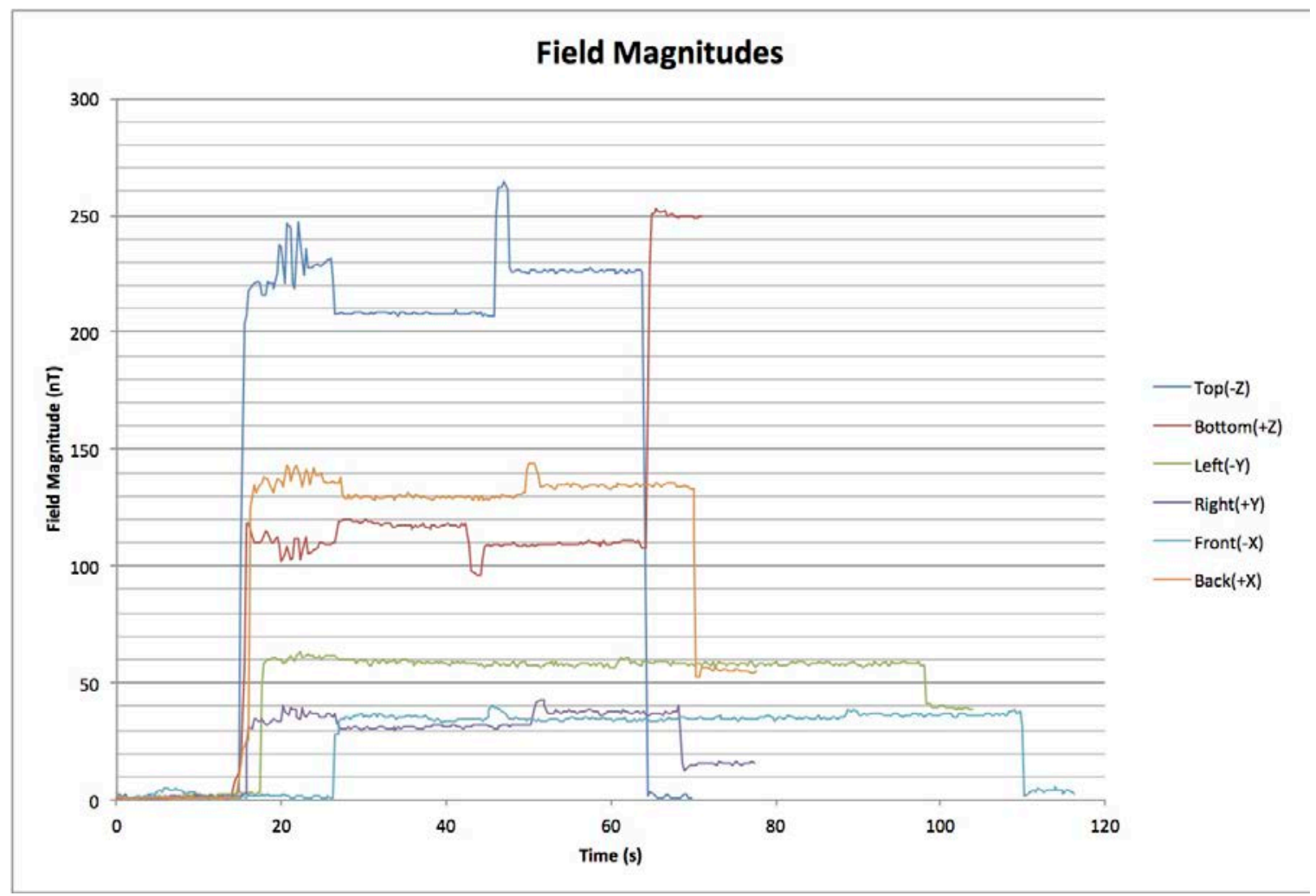

Figure 47: Active System Board Raw Field Magnitudes

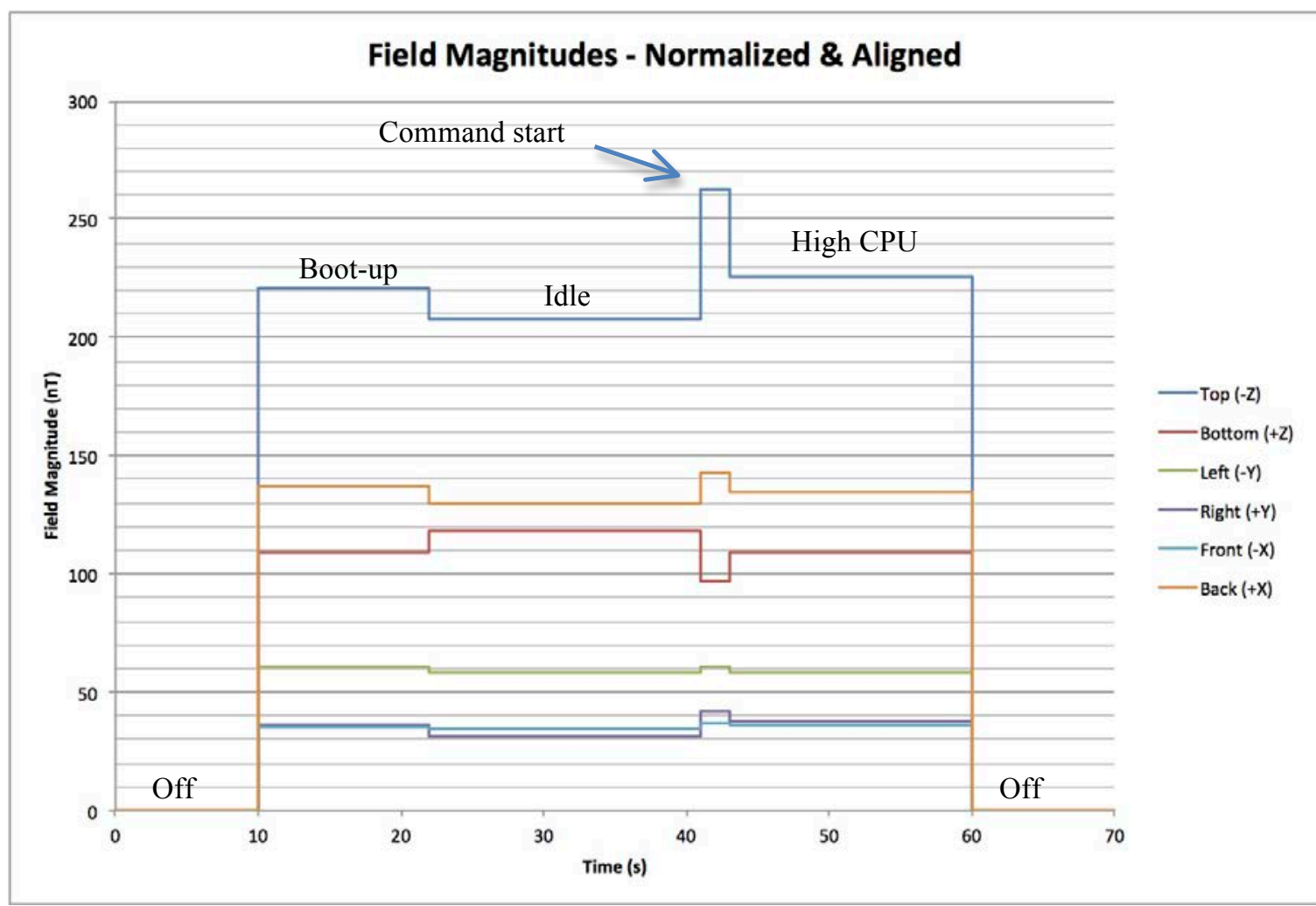

Figure 48: Active System Board Normalized and Aligned Field Magnitudes 
Table 3: Active Bus Field Strength Offsets Full Results in nT

\begin{tabular}{|c|c|c|c|c|c|c|c|c|}
\hline \multirow[t]{2}{*}{ Mode } & \multicolumn{4}{|c|}{ Top (-Z) } & \multicolumn{4}{|c|}{ Bottom (+Z) } \\
\hline & $X$-field & Y-field & Z-field & Mag. & $X$-field & Y-field & Z-field & Mag. \\
\hline Boot-up and login & -101 & -48 & 191 & 221 & 83 & 14 & 70 & 109 \\
\hline Idle & -99 & -26 & 181 & 208 & 86 & 16 & 79 & 118 \\
\hline Command engaged & -121 & -72 & 222 & 263 & 76 & 12 & 59 & 97 \\
\hline \multirow[t]{3}{*}{ CPU active } & -102 & -48 & 196 & 226 & 82 & 14 & 71 & 109 \\
\hline & \multicolumn{4}{|c|}{ Left $(-Y)$} & \multicolumn{4}{|c|}{ Right (+Y) } \\
\hline & X-field & Y-field & Z-field & Mag. & X-field & Y-field & Z-field & Mag. \\
\hline Boot-up and login & 44 & 41 & 10 & 61 & -23 & 11 & -25 & 36 \\
\hline Idle & 42 & 39 & 10 & 58 & -20 & 8 & -22 & 31 \\
\hline Command engaged & 48 & 37 & 5 & 61 & -27 & 12 & -30 & 42 \\
\hline \multirow[t]{3}{*}{ CPU active } & 44 & 37 & 7 & 58 & -24 & 10 & -27 & 37 \\
\hline & \multicolumn{4}{|c|}{ Front (-X) } & \multicolumn{4}{|c|}{ Back (+X) } \\
\hline & X-field & Y-field & Z-field & Mag. & X-field & Y-field & Z-field & Mag. \\
\hline Boot-up and login & -28 & -14 & -16 & 35 & -9 & -129 & -45 & 137 \\
\hline Idle & -28 & -15 & -15 & 35 & -9 & -123 & -40 & 129 \\
\hline Command engaged & -30 & -14 & -18 & 37 & -7 & -133 & -52 & 143 \\
\hline CPU active & -26 & -15 & -20 & 36 & -9 & -127 & -44 & 134 \\
\hline
\end{tabular}

Table 4: Active Bus Angle Errors Full Results in nT

\begin{tabular}{|c|c|c|c|c|c|c|}
\hline \multirow[t]{2}{*}{ Mode } & \multicolumn{3}{|c|}{ Top (-Z) } & \multicolumn{3}{|c|}{ Bottom (+Z) } \\
\hline & X-field & Y-field & Z-field & X-field & Y-field & Z-field \\
\hline Boot-up and login & 0.2 & 0.1 & 0.4 & 0.2 & 0.0 & 0.2 \\
\hline Idle & 0.2 & 0.1 & 0.4 & 0.2 & 0.0 & 0.2 \\
\hline Command engaged & 0.3 & 0.2 & 0.5 & 0.2 & 0.0 & 0.1 \\
\hline \multirow[t]{3}{*}{ CPU active } & 0.2 & 0.1 & 0.4 & 0.2 & 0.0 & 0.2 \\
\hline & \multicolumn{3}{|c|}{ Left (-Y) } & \multicolumn{3}{|c|}{ Right (+Y) } \\
\hline & X-field & Y-field & Z-field & X-field & Y-field & Z-field \\
\hline Boot-up and login & 0.1 & 0.1 & 0.0 & 0.1 & 0.0 & 0.1 \\
\hline Idle & 0.1 & 0.1 & 0.0 & 0.0 & 0.0 & 0.1 \\
\hline Command engaged & 0.1 & 0.1 & 0.0 & 0.1 & 0.0 & 0.1 \\
\hline \multirow[t]{3}{*}{ CPU active } & 0.1 & 0.1 & 0.0 & 0.1 & 0.0 & 0.1 \\
\hline & \multicolumn{3}{|c|}{ Front $(-X)$} & \multicolumn{3}{|c|}{ Back $(+X)$} \\
\hline & X-field & Y-field & Z-field & X-field & Y-field & Z-field \\
\hline Boot-up and login & 0.1 & 0.0 & 0.0 & 0.0 & 0.3 & 0.1 \\
\hline Idle & 0.1 & 0.0 & 0.0 & 0.0 & 0.3 & 0.1 \\
\hline Command engaged & 0.1 & 0.0 & 0.0 & 0.0 & 0.3 & 0.1 \\
\hline CPU active & 0.1 & 0.0 & 0.0 & 0.0 & 0.3 & 0.1 \\
\hline
\end{tabular}




\section{CONCLUSION}

Performance of the Standalone HMC5883L

Based on the testing performed on the standalone HMC5883L development board it is apparent that the magnetometer chip does not come with any pre-established calibration. Luckily it is relatively straightforward to analyze the reported magnetic field readings and compare them with a known magnetic field to produce reliable calibrated values. The process consists of placing the magnetometer in the center of a calibrated Helmholtz cage, applying known magnetics fields, and plotting the known field against the reported field. A simple linear trend line extrapolation will yield a slope-intercept form equation that can be used to convert the field values given by the HMC5883L into calibrated magnetic field data.

The testing performed in the development of this paper utilized a homemade Helmholtz cage magnetic simulator. Although not a perfect design, it was sufficient to establish the process of calibration. In the end, it could be shown that the HMC5883L magnetometer was able to produce magnetic field vectors with average accuracy on the order of $0.2^{\circ}$ in a magnetic field strength of $50000 \mathrm{nT}$.

\section{Effect of CubeSat Electronics}

In addition to developing a method for calibrating the HMC5883L magnetometer for use in Cal Poly's CubeSat projects, the effect of spacecraft electronics on the magnetometer was investigated as well. Due to the early stage of development of the new Cal Poly CubeSat bus, only the primary electronics board, or system board, could be tested. Results show the system board induces a noticeable magnetic field when active, however 
increased or decreased computational activity and power draw is negligible in comparison. Therefore active electronics result in a constant magnetic offset which can be subtracted.

\section{Future Work}

While the Helmholtz cage used in this project was sufficient to provide useful results on a single magnetometer chip, it is recommended a larger, more professionally built cage be used for future flight CubeSat testing. Future CubeSat missions utilizing the $3 \mathrm{U}$ form factor will absolutely require a larger cage due to the size of the CubeSat bus with respect to the Helmholtz cage volume.

Future missions requiring increased accuracy in magnetic sensing will also need to investigate the effects of solar panel and power system electrical current. Since the HMC5883L chips are mounted to the inside of the solar cell carrying boards, fluctuating sunlight due to Earth eclipsing and satellite rotation will cause fluctuating currents to pass through traces adjacent to the magnetometers. The magnetic field offsets will need to be measured as a function of solar cell current so that active calibration can occur on orbit to subtract solar cell effects out. An additional expected source of magnetic noise not tested due to unavailability of hardware is that due to RF interference. This source will need to be incorporated into future calibration work as well due to the high data throughput expected on future missions. 


\section{REFERENCES}

[1] Cal Poly CubeSat. “CubeSat Design Specification, Rev 12.” August 2009, available online at "http://www.cubesat.org/." Accessed 26 Aug 2012.

[2] Bland, Ivan. "Receive Sensitivity Characterization of the PolySat Satellite Communication System.” Cal Poly Masters Thesis. March 2010.

[3] Honeywell. "HMC105X Datasheet." January 2010. Available online at “http://www.honeywell.com/" Accessed 1 Jul 2012.

[4] Fortosis, Nick. "Testing and Debugging the B-Dot Control System for CP-6." Cal Poly Engineering REU Program. August 2009.

[5] Honeywell. "HMC5883L Datasheet." April 2012. Available online at “http://www.honeywell.com/.” Accessed 1 Jul 2012.

[6] Honeywell. "Applications of Magnetic Position Sensors." Available online at “http://www.honeywell.com/.” Accessed 8 Sep 2012.

[7] Meeker, David. "Finite Element Method Magnetics." Available online at “http://www.femm.info/.” Accessed 7 Sep 2012. 


\section{APPENDICES}

\section{A. Building the Coils}

Helmholtz coils are a simple concept and therefore can be built using a wide variety of materials. If stiff enough wire is used, an external structure may not even be required except to stand and position the coils in the right place. For durability, however, I chose to enclose each coil within a plastic cover. After researching wire and cable covers I selected a gray plastic covered cable-mounting tray, one inch in thickness and width, from cableorganizers.com. These trays are normally used to route wires and cabling around homes and offices, or behind desks or entertainment systems.

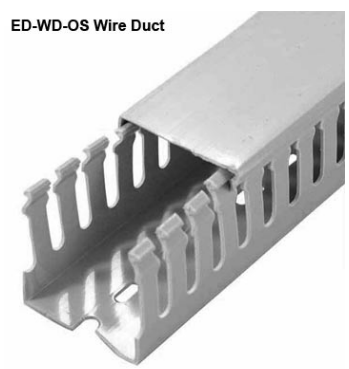

The benefits of these cable trays include low cost, strength, pre-drilled holes for mounting, side-slots for running wire, and the ability to bend and shape with relatively low heat. They are thick and wide enough to be wrapped with hundreds of turns of high gauge magnet wire, and at two meters in length each, can make one-piece Helmholtz coils at up to 25 inches in diameter. If larger diameters are required, two pieces can be easily combined by offsetting the break in the bottom from the break in the snap-on cover.

Construction of the coils can begin after selection of the small, medium and large sizes has been made using the previously mentioned formulas. The next step is to make 
molds around which the cable trays can be bent. I used a circular drilling tool and plywood scraps from the hardware store to make three wooden circles corresponding to the diameters of the three sizes of coils.

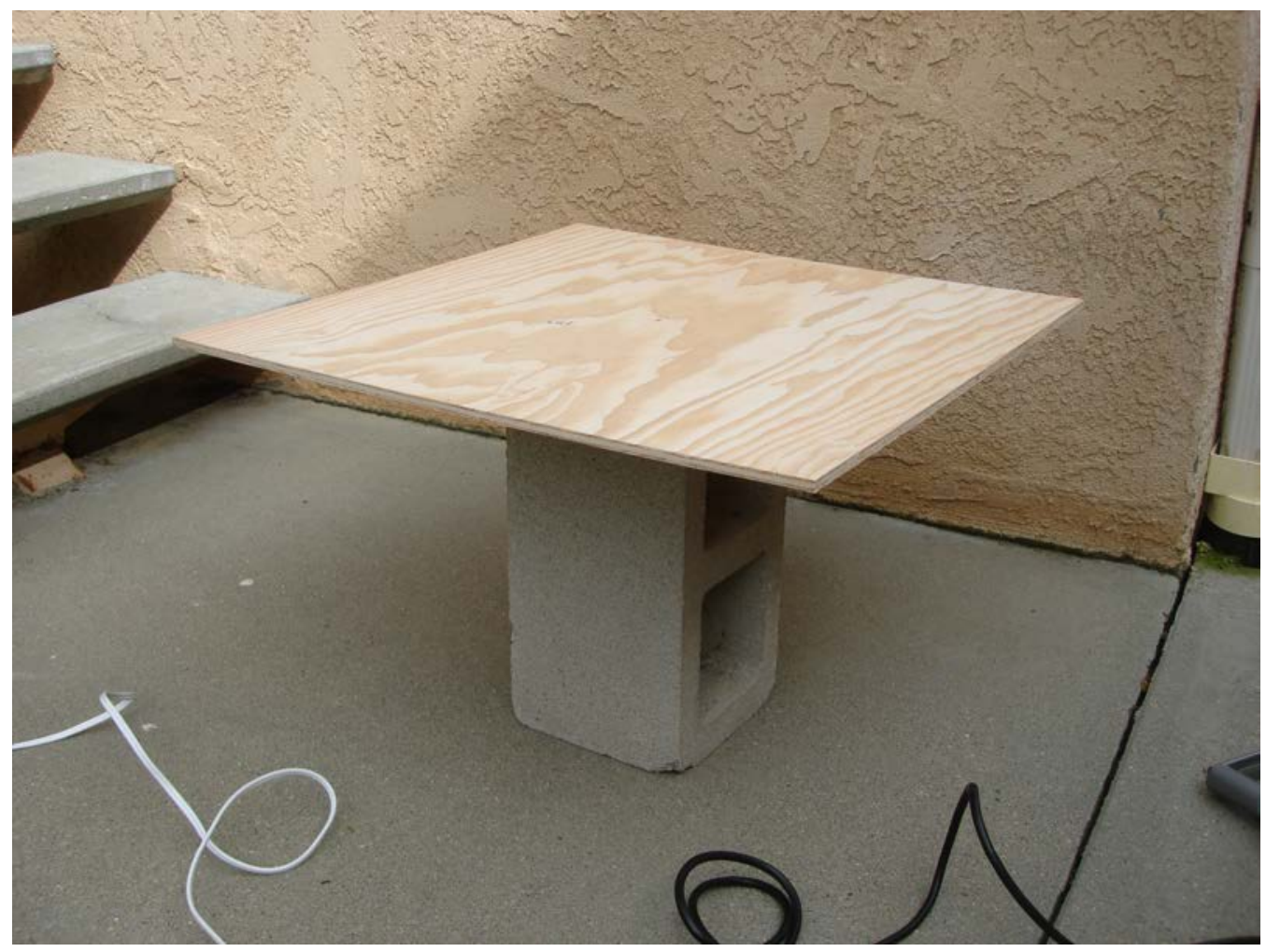

Plywood scrap from hardware store 


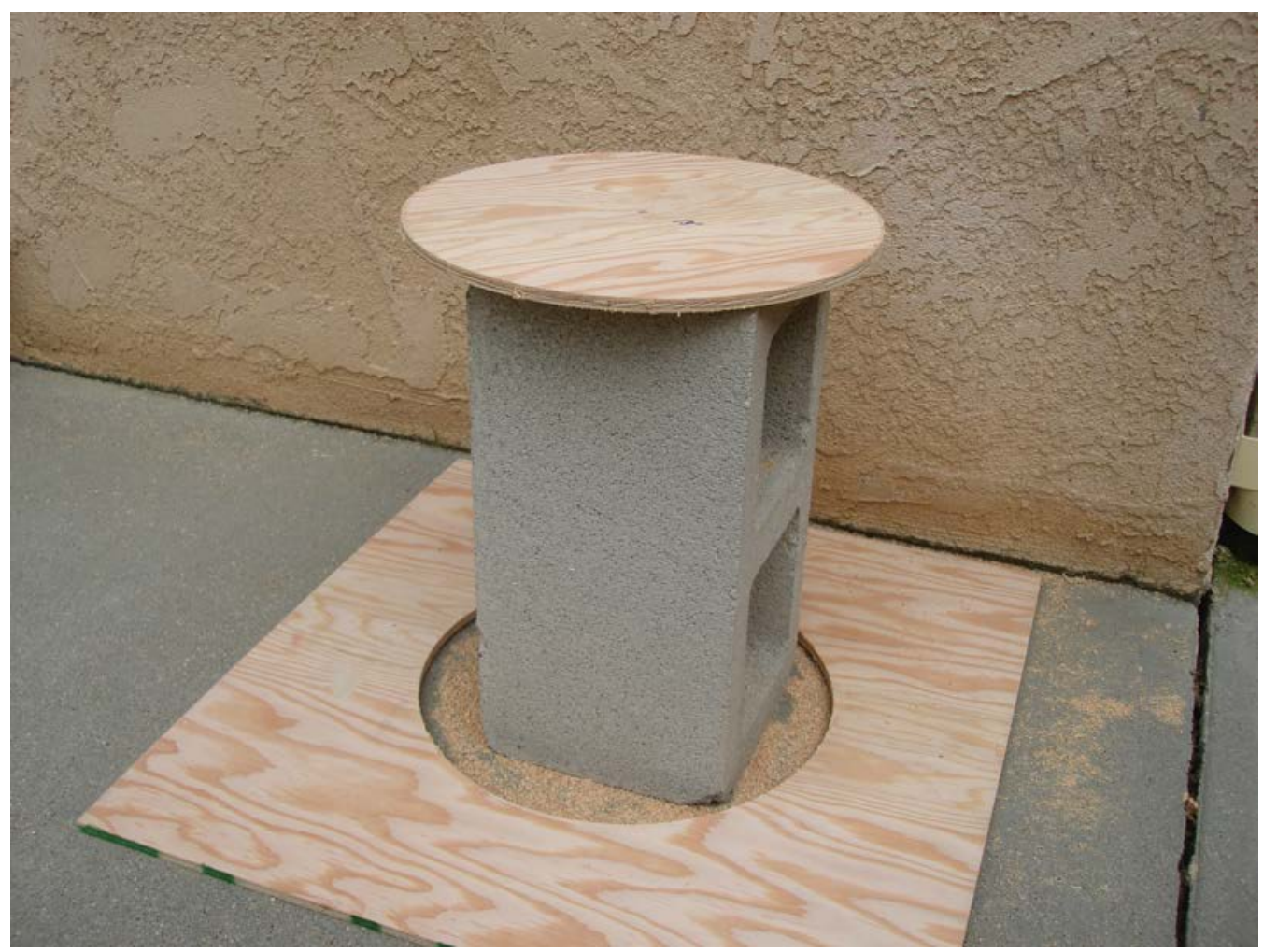

Use circular cutting tool to cut the wooden circles of the three diameters

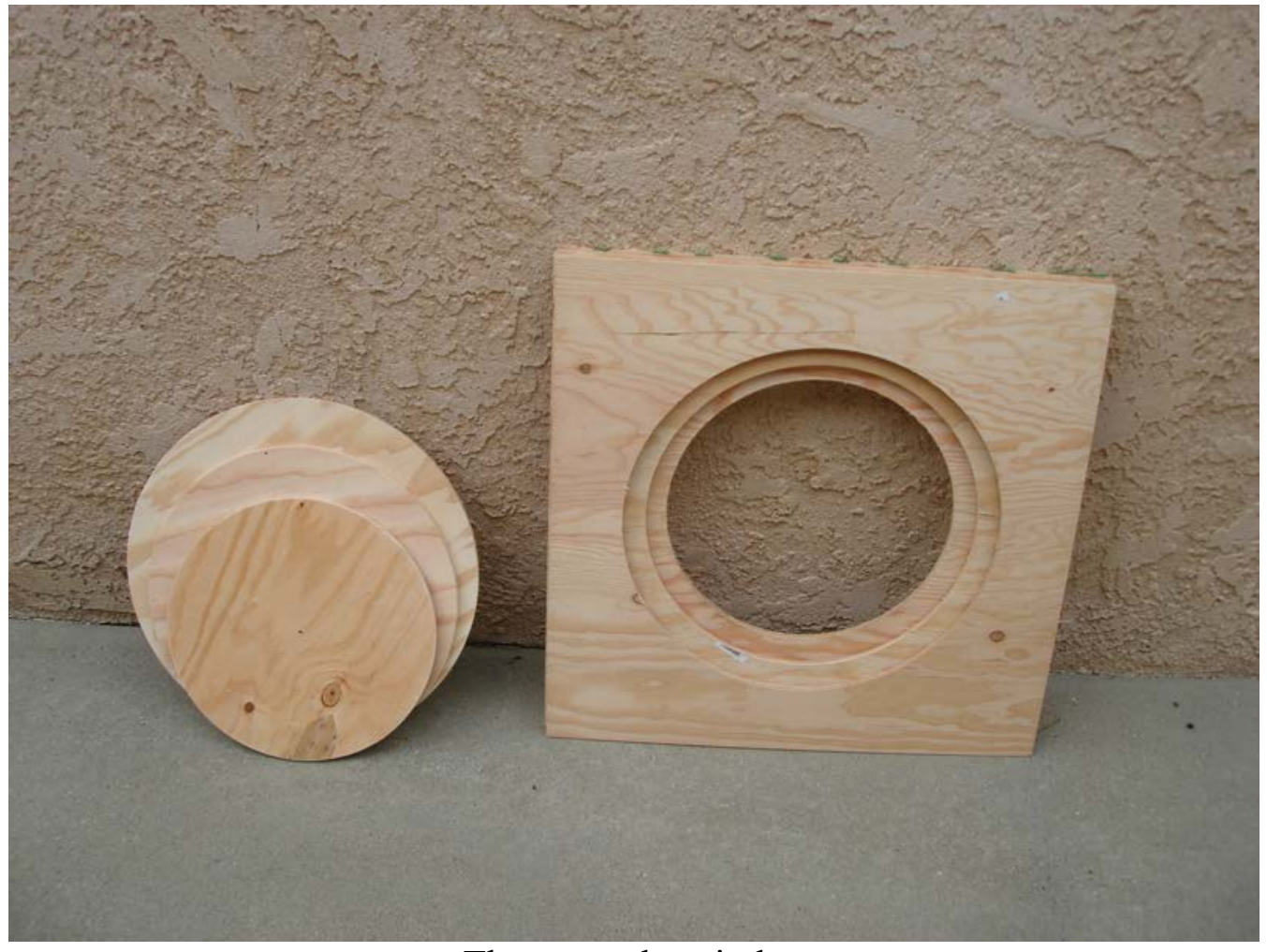

Three wooden circles 
After the molds are cut, they can be used to bend the cable trays to appropriate sizes. A wide, flat workspace is extremely helpful for this step, as working with circular objects can get awkward. My setup consisted of the dining room table, a stack of paper, the mold, and a weight. Because of the low heat required to bend these trays, all I needed was a standard hairdryer to accomplish the task. However, other cable trays or materials might require higher heat, in which case a workbench with higher heat tolerances would be a better choice than the dining room table.

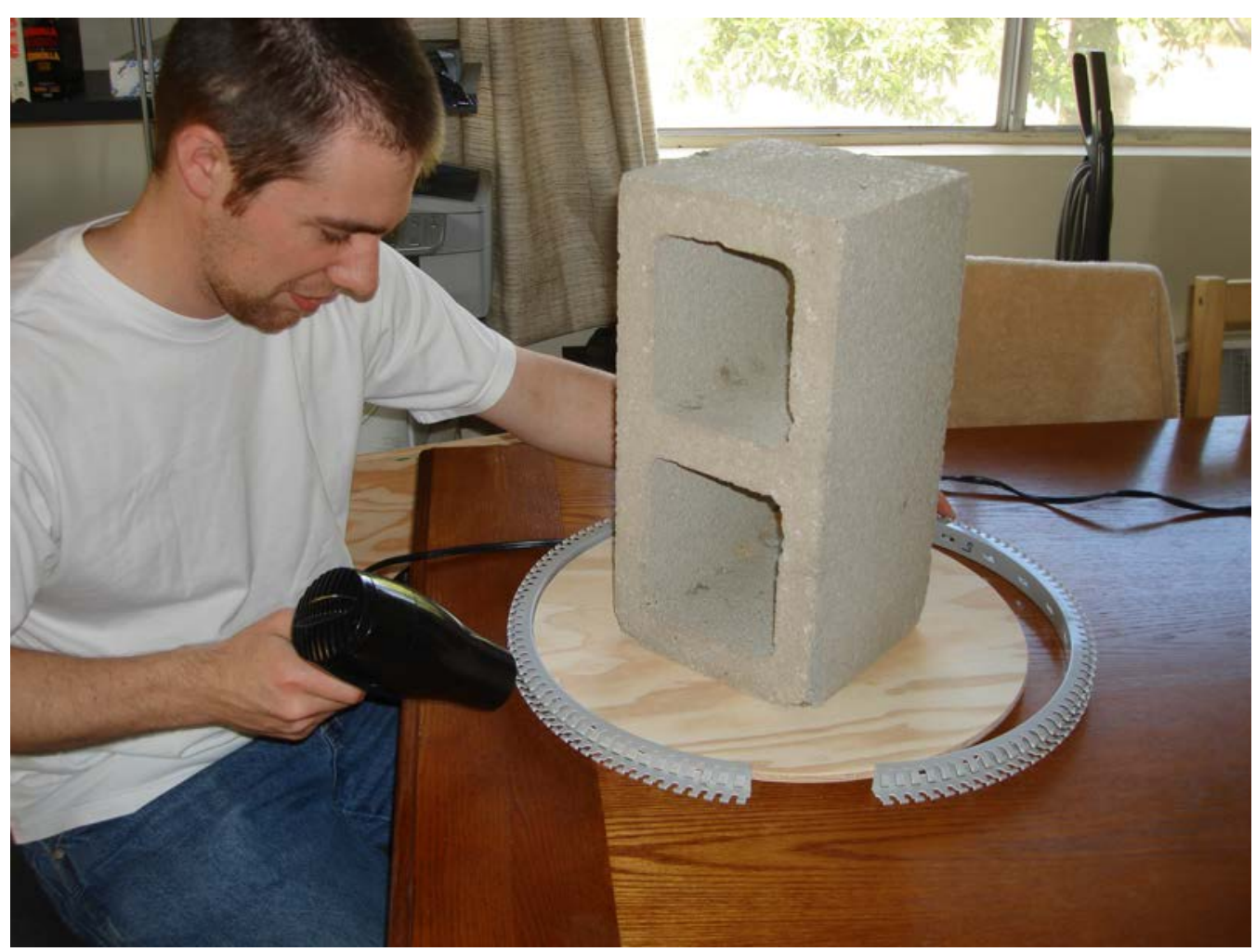

Bending the cable tray

To bend the tray, the wooden mold is placed on top of the stack of paper so the mold is in the middle of the cable tray vertically when placed flat on the work surface. The weight is placed on top of the mold to keep it from moving during the bending 
process. The length of tray required for the desired diameter can be estimated using the formula for circumference of a circle, though an extra inch or two should be added as margin as the tray can sometimes stretch or shrink in the bending process. Using a pushpin or thumbtack, one end of the tray is attached to the side of the mold, and then applying heat with the hairdryer at the bending point, the tray is continuously bent around the circle until the two ends are nearly touching. At that point the end can be cut to exactly the right length with a utility knife or wire cutters, and bent down under heat to complete the circle.

If the cover is to be used, the same process is repeated with the end of the cover attached to the bent tray, then heated and bent around the circle. With both the tray and the cover, bending can cause the sides to bow in or out. While the side facing the work surface should be held flat, the top can be guided by hand if it starts to deflect. It is recommended to wear gloves for this even for hairdryer heat. If a part has become undesirably deflected, it can be reheated and corrected. Once cooled, it should be stiff and maintain its shape.

After this process has been repeated for all coils, all the bending equipment can be removed and put away. Back on the work surface, a bent tray is placed down flat and the cover is removed from around it. Using a strong tape like duct tape or packaging tape, connect the cut ends together so the circle is structurally whole.

The next step in creating the coils is to wrap magnet wire around the circular cable trays. This is done by attaching one end of the magnet wire to one of the slots in the cable tray, then wrapping it around as many times as was decided using the turns variable of the previous formula for field strength of a Helmholtz coil. 


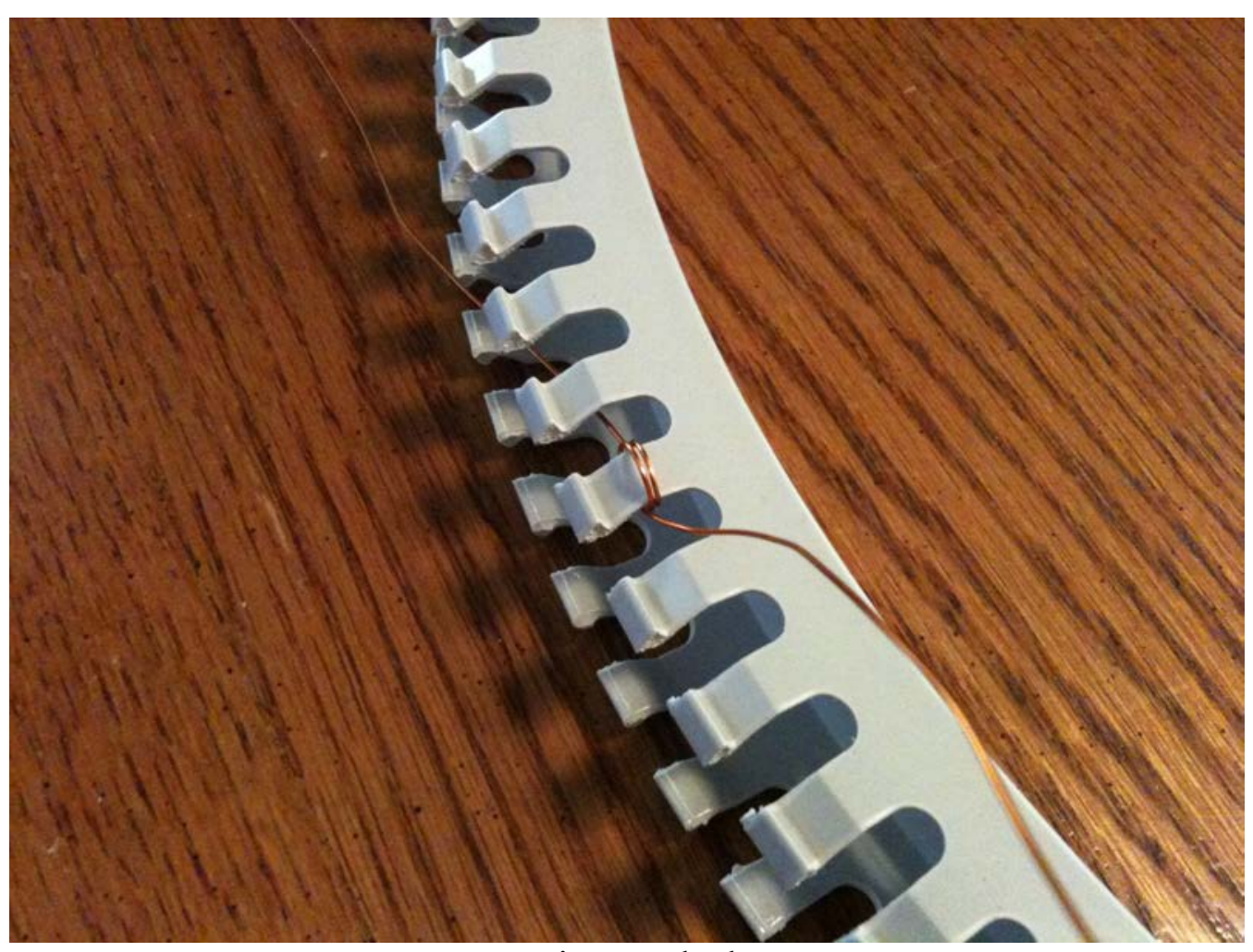

Magnet wire attached to tray

Having the spool of magnet wire held by a friend or a wire dispenser can greatly assist in the wrapping process. Once the total number of turns is completed, the magnet wire can be cut from its spool and wrapped around a tray slot next to the starting end and in the same fashion. This process is repeated for each of the six coils. 


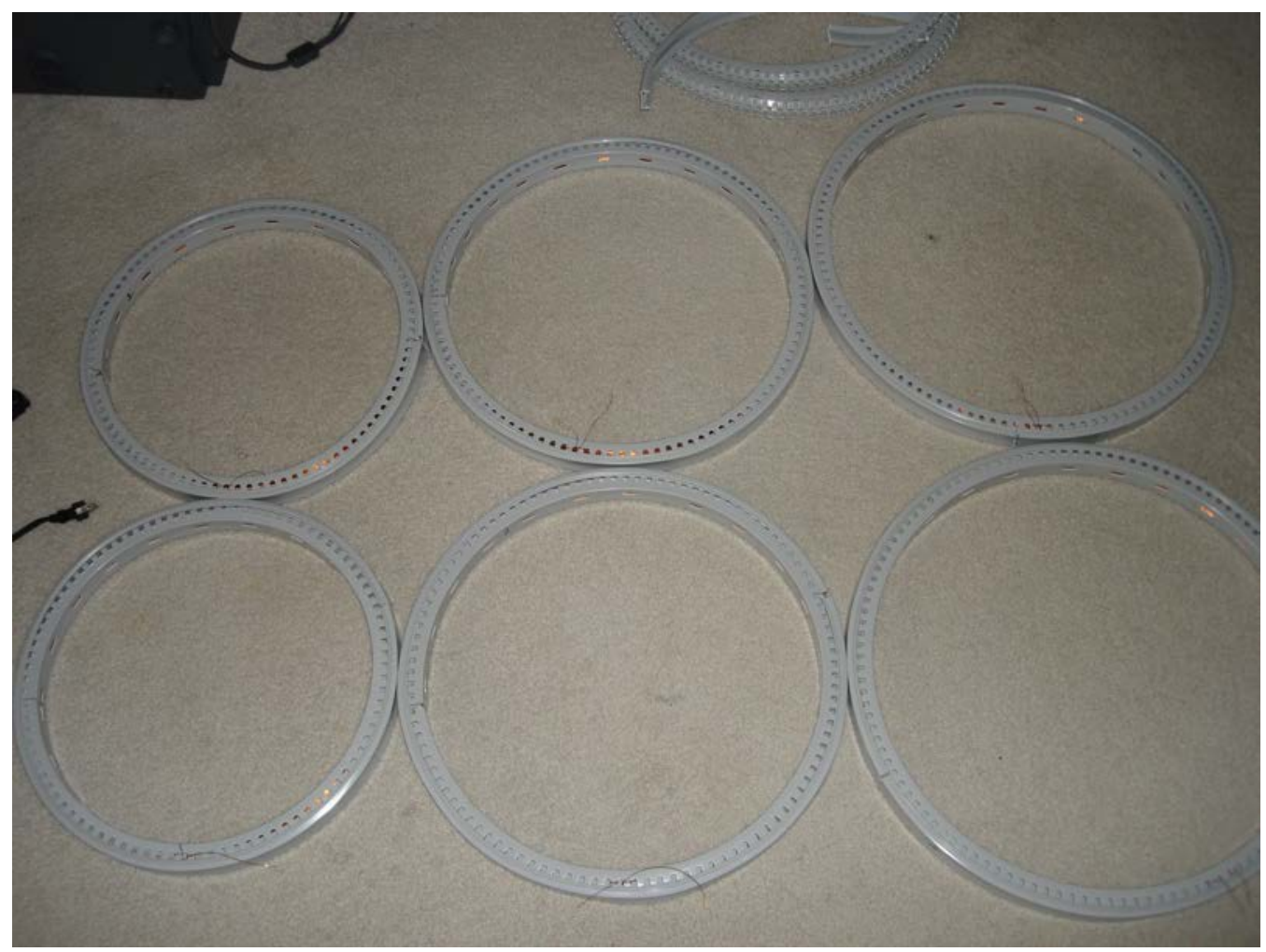

Six bent and wrapped coils

The cage assembly can be constructed using the six completed coils and four wooden dowels. The wooden dowels act as legs to support the assembly on top of a flat surface.

The most important part in assembling the cage is keeping all the parallel coils as parallel as possible and the orthogonal coils as orthogonal as possible. Starting with the smallest set of coils, four marks are made at equal distances around the circumference to indicate the attachment points of the wooden dowels. This should be done while keeping in mind the electrical connection points should end up at the same position when the coils are stacked on top of each other. The wooden dowels are measured and cut such that they span the distance of the coils' combined thicknesses, plus their separation equal to their radius, plus the distance from the bottom face of the bottom coil to the surface the cage will be placed on. That value should be calculated based on the height of the air bearing 
base underneath it and the other two sets of coils that will be perpendicularly attached with coexisting center points. All of these measurements should be marked on the dowel for straight alignment of the coils.

One of the small coils is placed flat on the work surface and a cardboard box or similar square object is placed on top of it with a corner at one of the equidistant marks made earlier. The first wooden dowel is placed with one end on the work surface and touching both the mark on the coil and the parallel corner of the box to keep it straight. Hot glue is applied to attach it to the coil and the process is repeated for each of the other three wooden dowels. Using the other marks on the dowels as a guide, the second small coil is attached with hot glue at a distance equal to one radius away from the first coil.

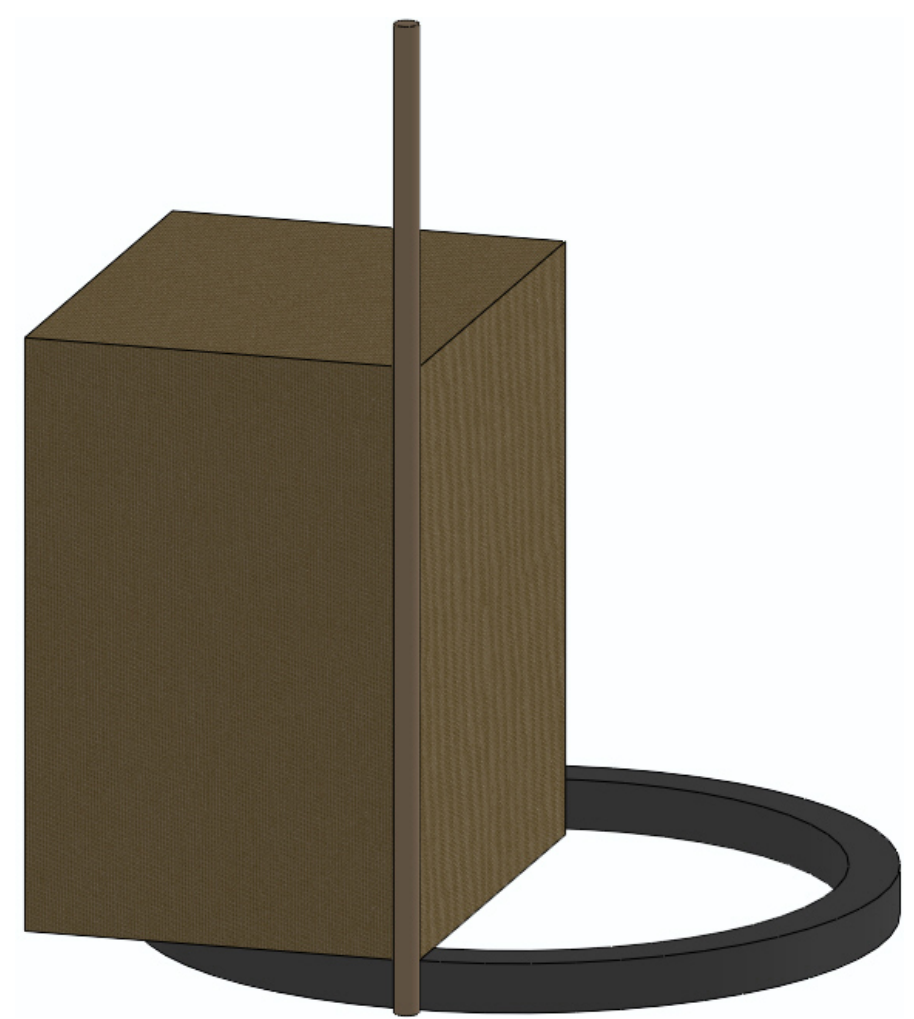

Alignment Apparatus 


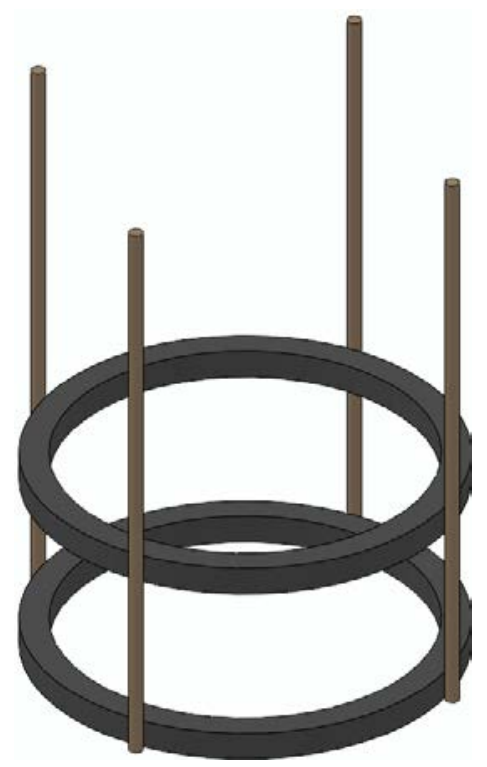

Small coils attached

Once the two small coils (Z-axis) are attached to the wooden dowels, the fixture can be flipped right-side up. The two perpendicular coil sets (Y-axis and $\mathrm{X}$-axis) should then easily slide on and glue at the mate points.

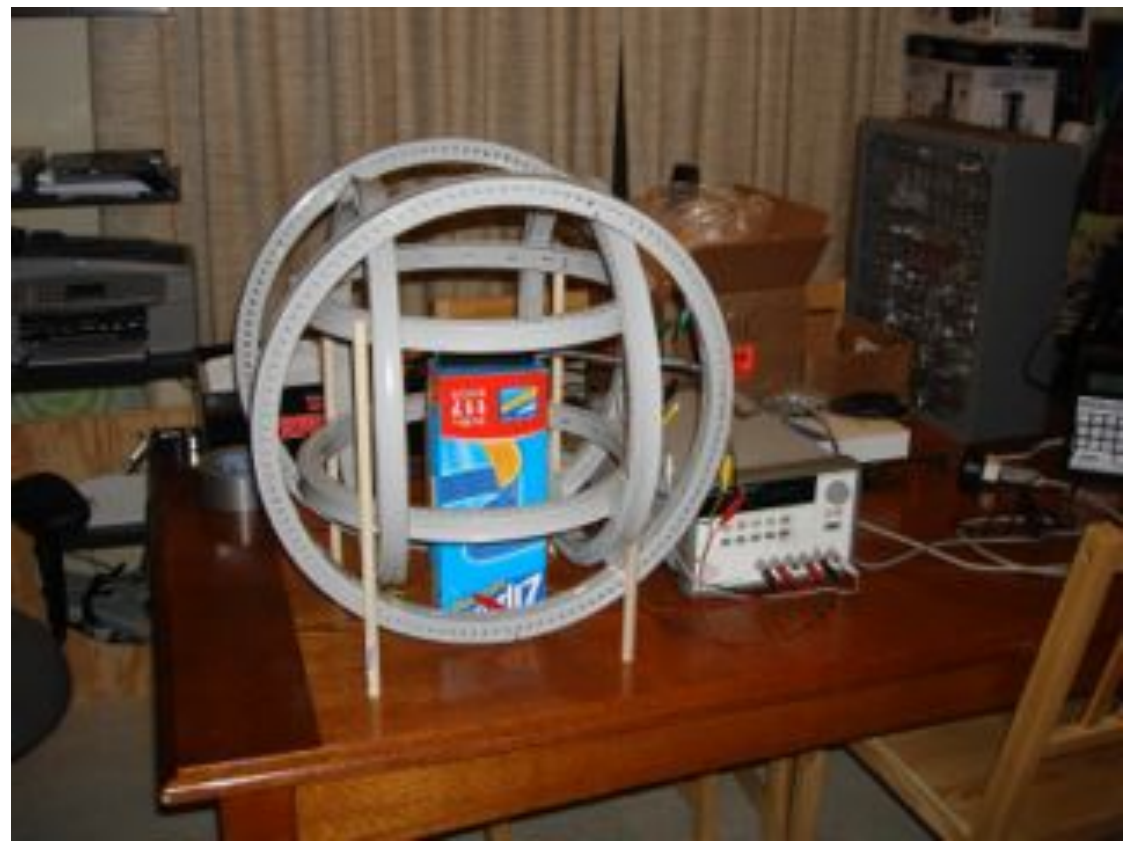

All 6 coils attached 
Driving the Coils

A computer is required to run long-term simulations of dynamic magnetic fields, and will be responsible for computing the magnetic field at any given point around Earth, then applying that field to the Helmholtz cage. My software environment of choice is LabVIEW because of its integration of hardware control and my experience with the software. I am using an Agilent E3631A triple output power supply because it's available in the PolySat lab, it has computer connectivity built in, and with three outputs, only one power supply is required. Unless the power supply is able to provide forward and reverse current automatically, an additional polarity control box is required to provide full magnetic field range. Each coil must be capable of producing a field in both the positive and negative direction. For my setup, I assembled a simple parallel port controlled relay box. Each of the three relays, when actuated, reverses the polarity of the coil it is connected in line with. Because it is parallel port controlled, it can be connected to the same computer as the power supply and automatically switch when needed.

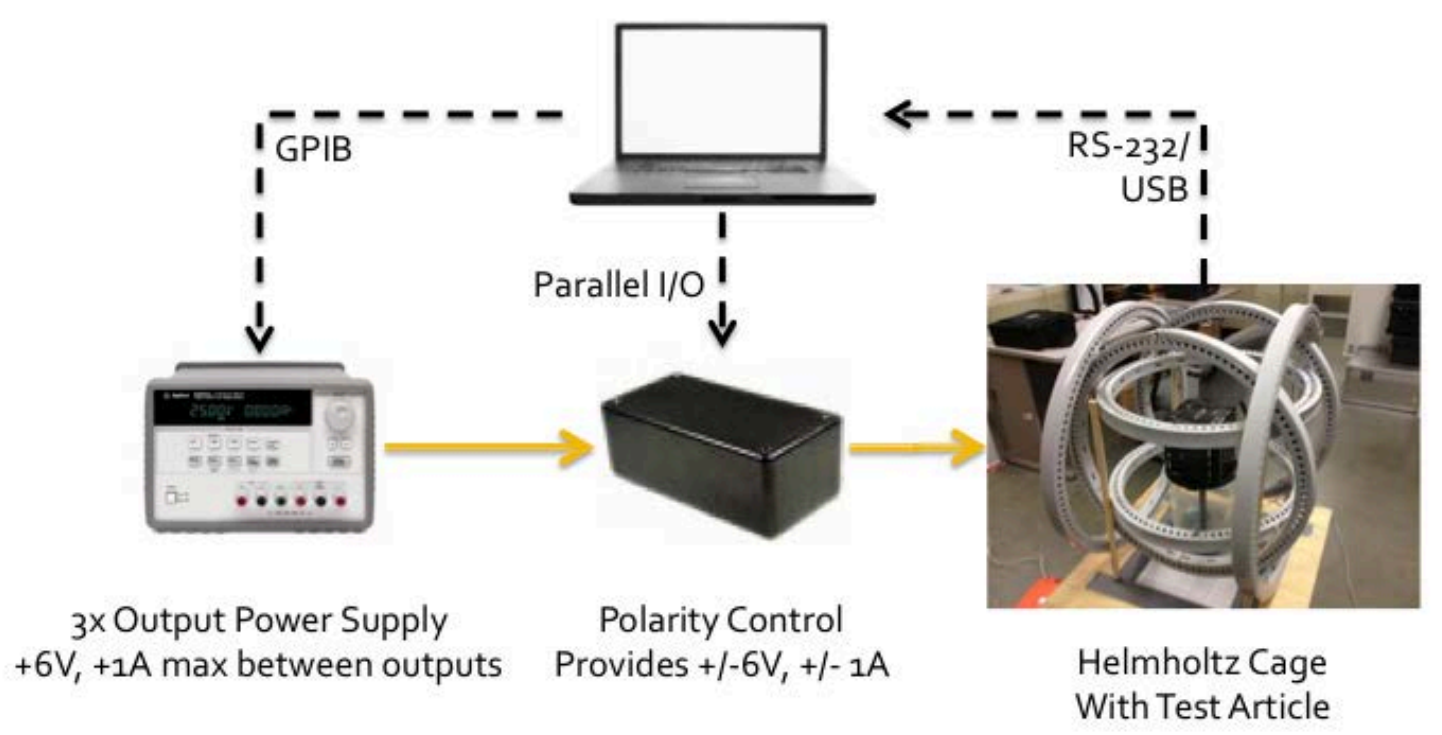




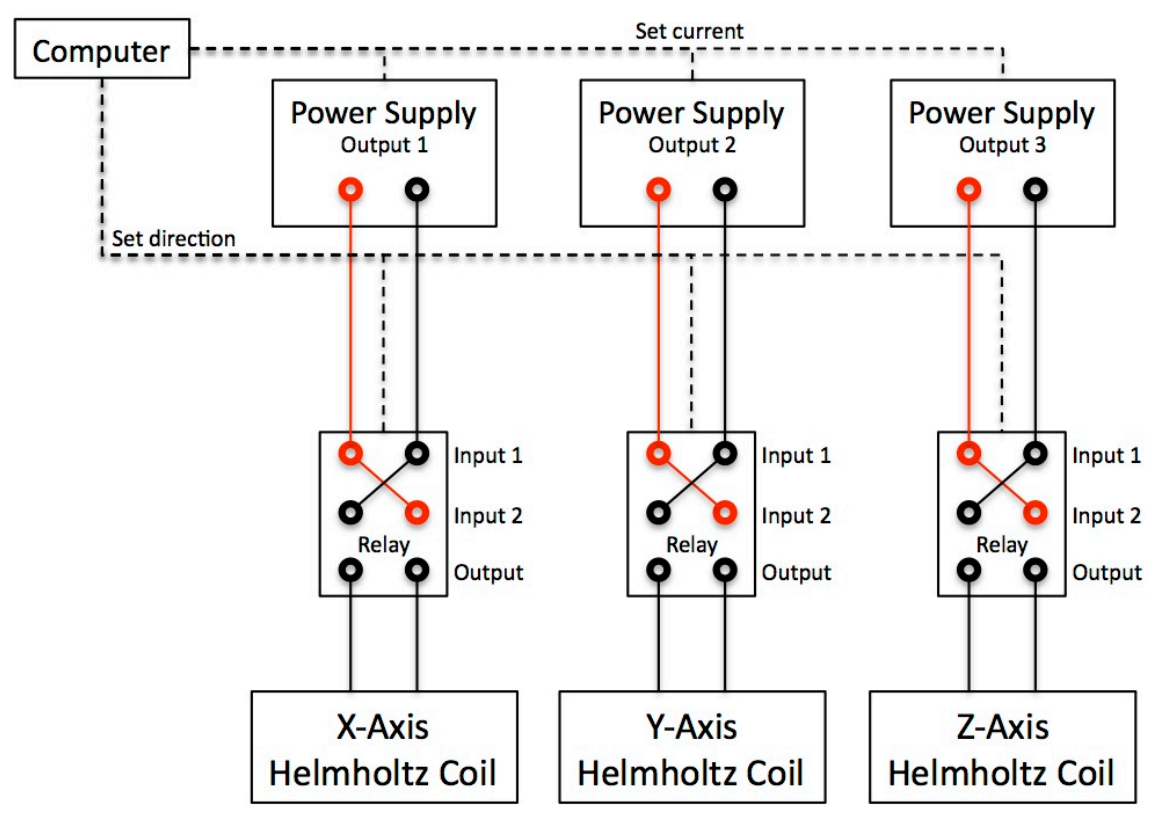

There are two additional software packages required to make magnetic field computations. One is for finding the position of a spacecraft at any point in its orbit in a specified time period. The other is for determining the magnetic field direction and magnitude at that location. For the former I chose a free open-source program called Predict. For the latter I chose a free program available from the National Geophysical Data Center, NOAA website called WMM2010. Both programs run from the Windows command line and therefore can easily be executed from within a LabVIEW program.

Simulation of a magnetic field in the Helmholtz cage requires four steps: Provide a text file containing the two-line elements of a satellite you want to simulate, determine the time period you want magnetic field data for, enter this data into the WMM Get.vi LabVIEW program and run it, open the resulting file in the Mag Field Simulator.vi program and run it. 
B. LabVIEW Programs

Set Coil Current.vi

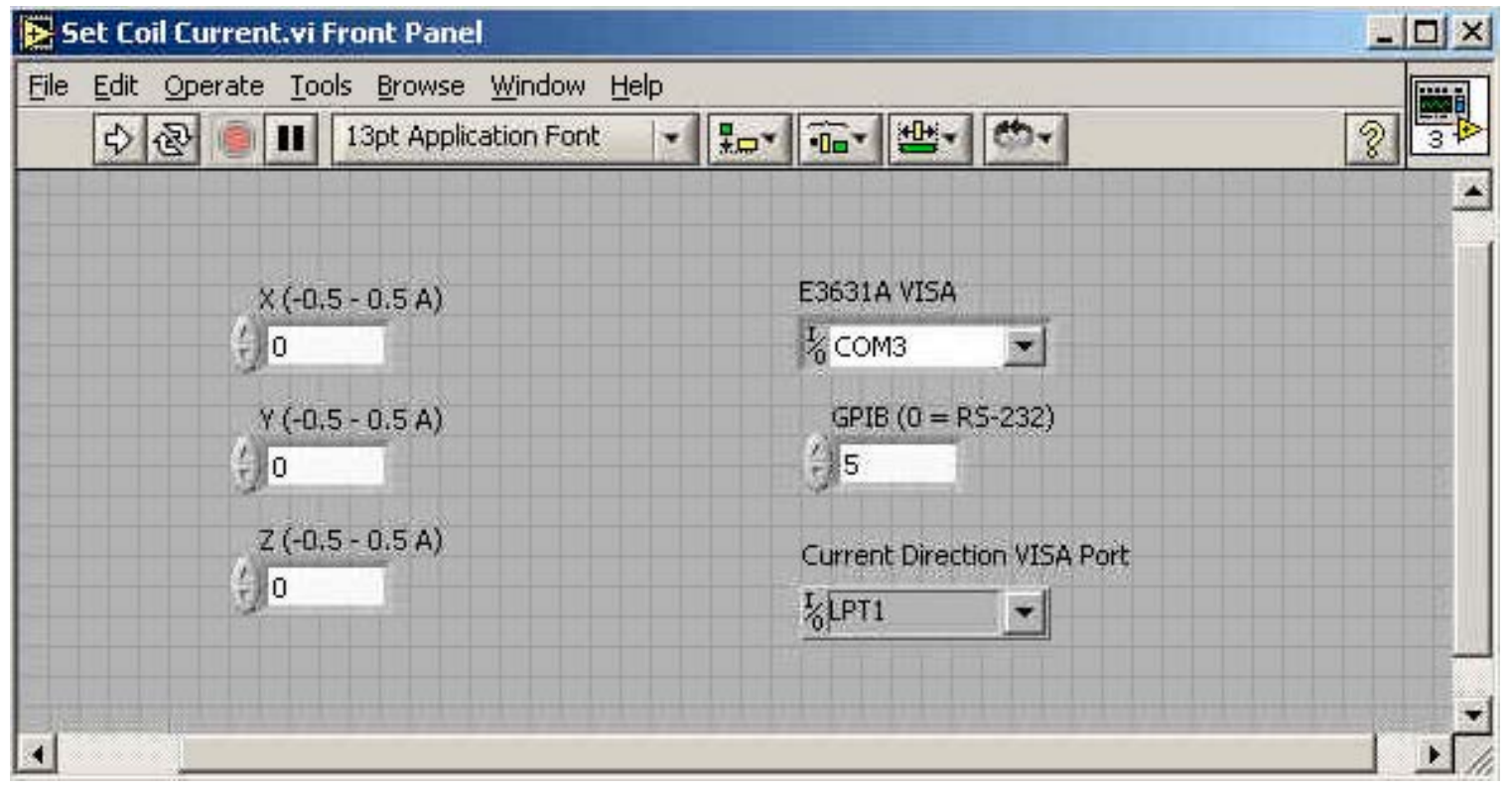

Set Coil Current uses the most basic functions of LabVIEW to talk directly with the E3631A power supply and polarity switch box to induce desired currents in each set of coils. 


\section{Coil Calibration Curve.vi}

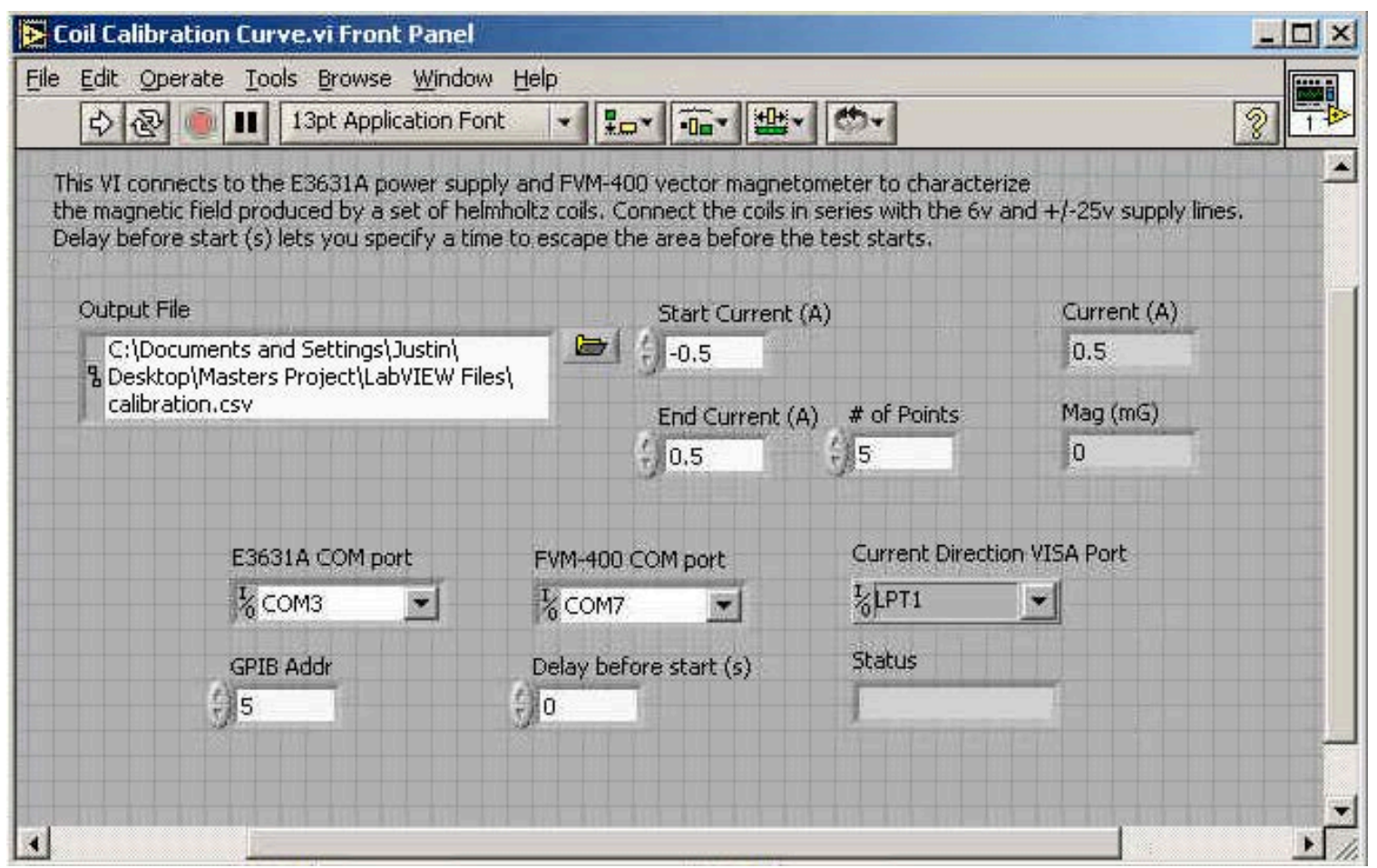

Coil Calibration Curve is required for all other Helmholtz control VI's and is the first step for any calibration procedures. Using "Set Coil Current.vi", it induces a current sweep through each coil axis according to the specified values and reads the resulting magnetic fields from the FVM-400 vector magnetometer. Using the two sets of information it then produces the calibration coefficients that relate a desired magnetic field vector to currents required to send to "Set Coil Currents.vi" and saves them in a calibration file. 


\section{Set Mag Field Vector.vi}

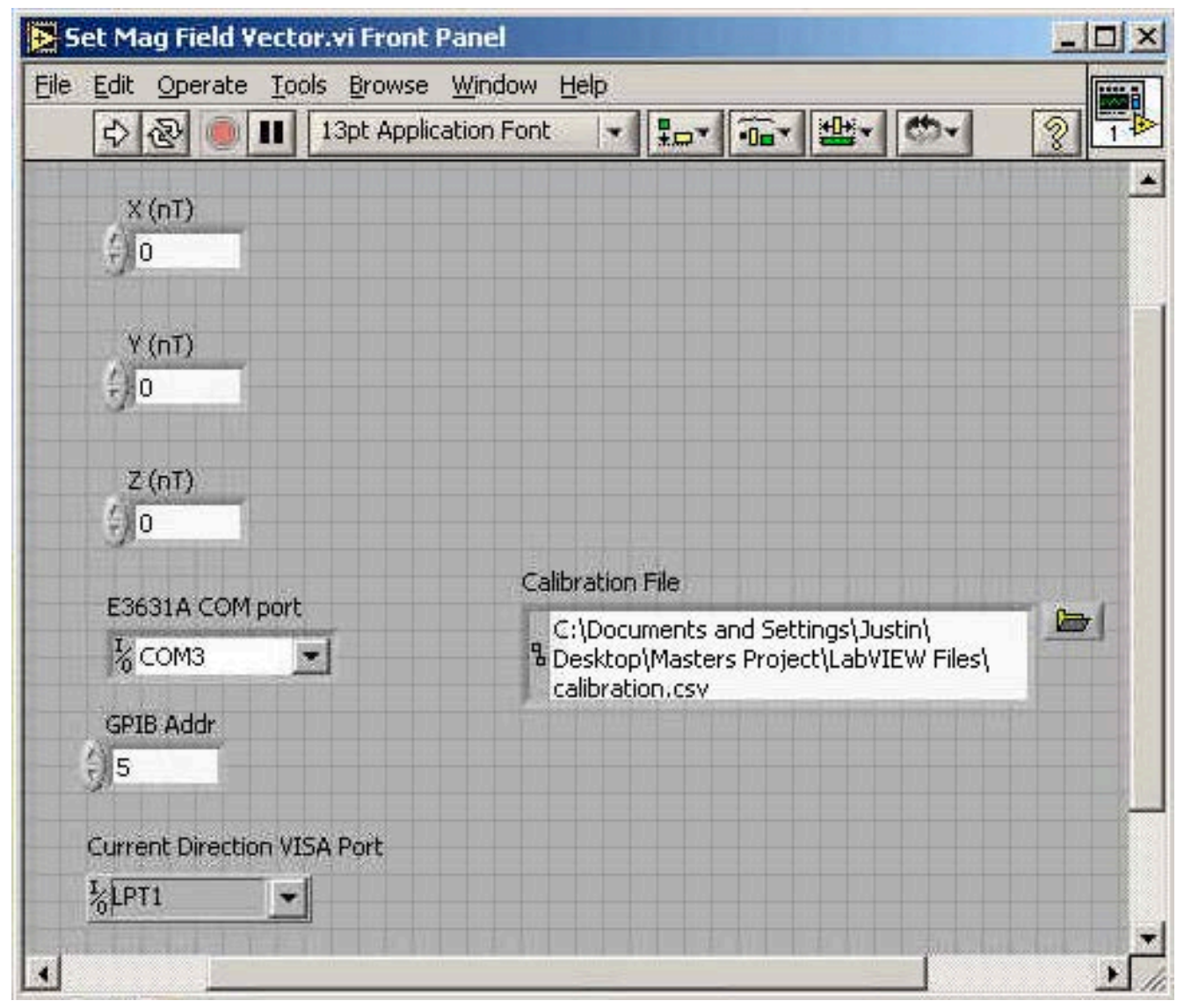

Set Mag Field Vector uses the calibration values from Coil Calibration Curve to induce the desired magnetic field vector in the Helmholtz cage. 


\section{Mag Field Simulator.vi}

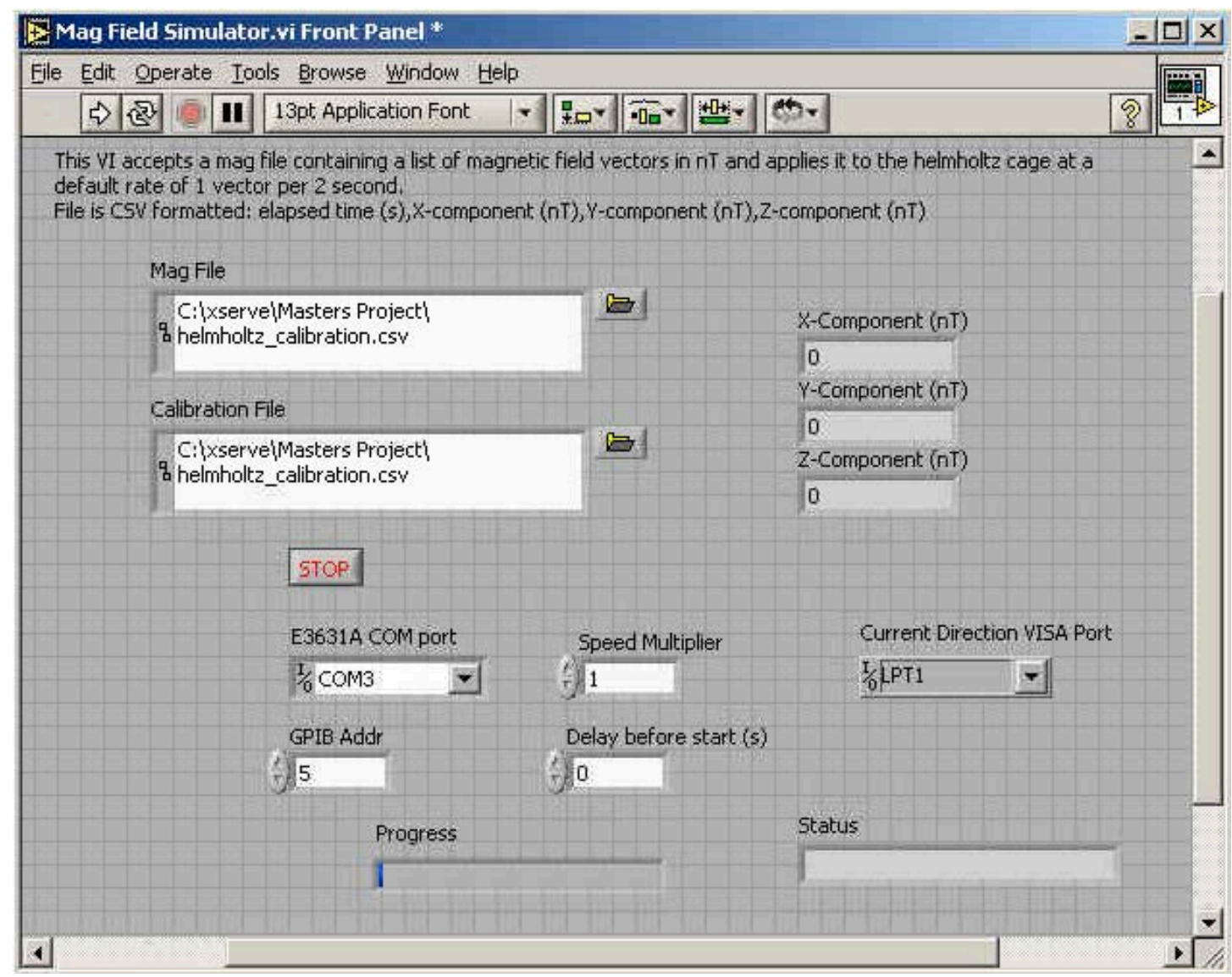

Mag Field Simulator takes a CSV file of magnetic field vectors and induces each one in sequence to the Helmholtz cage. 
Field sim and mag reader.vi

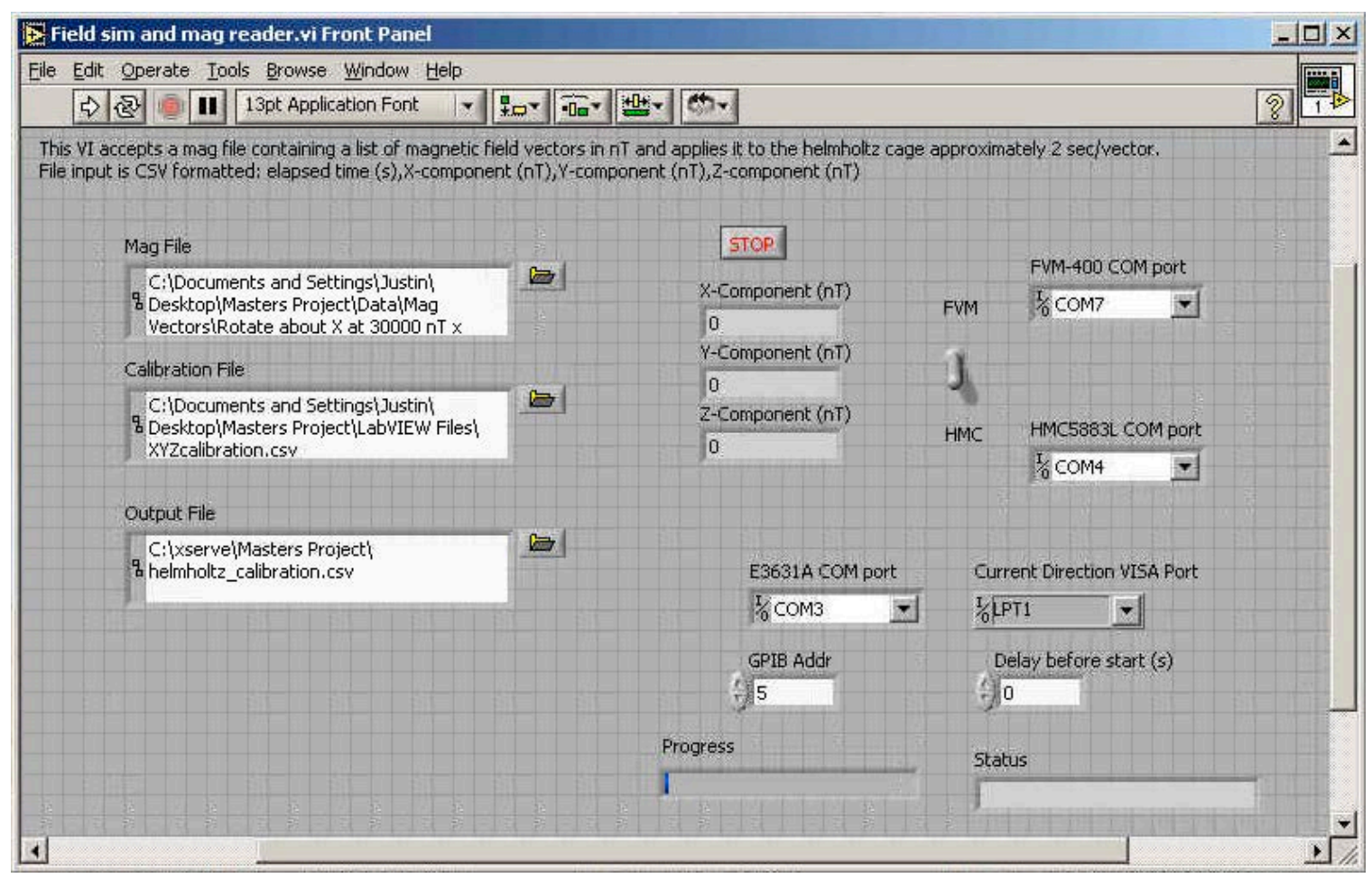

Field sim and mag reader performs the same task as Mag Field Simulator plus the added functionality of taking magnetic field measurements after each vector is applied to the Helmholtz cage. This is used in the magnetometer calibration process to compare actual magnetic field to sensor-reported magnetic field. 


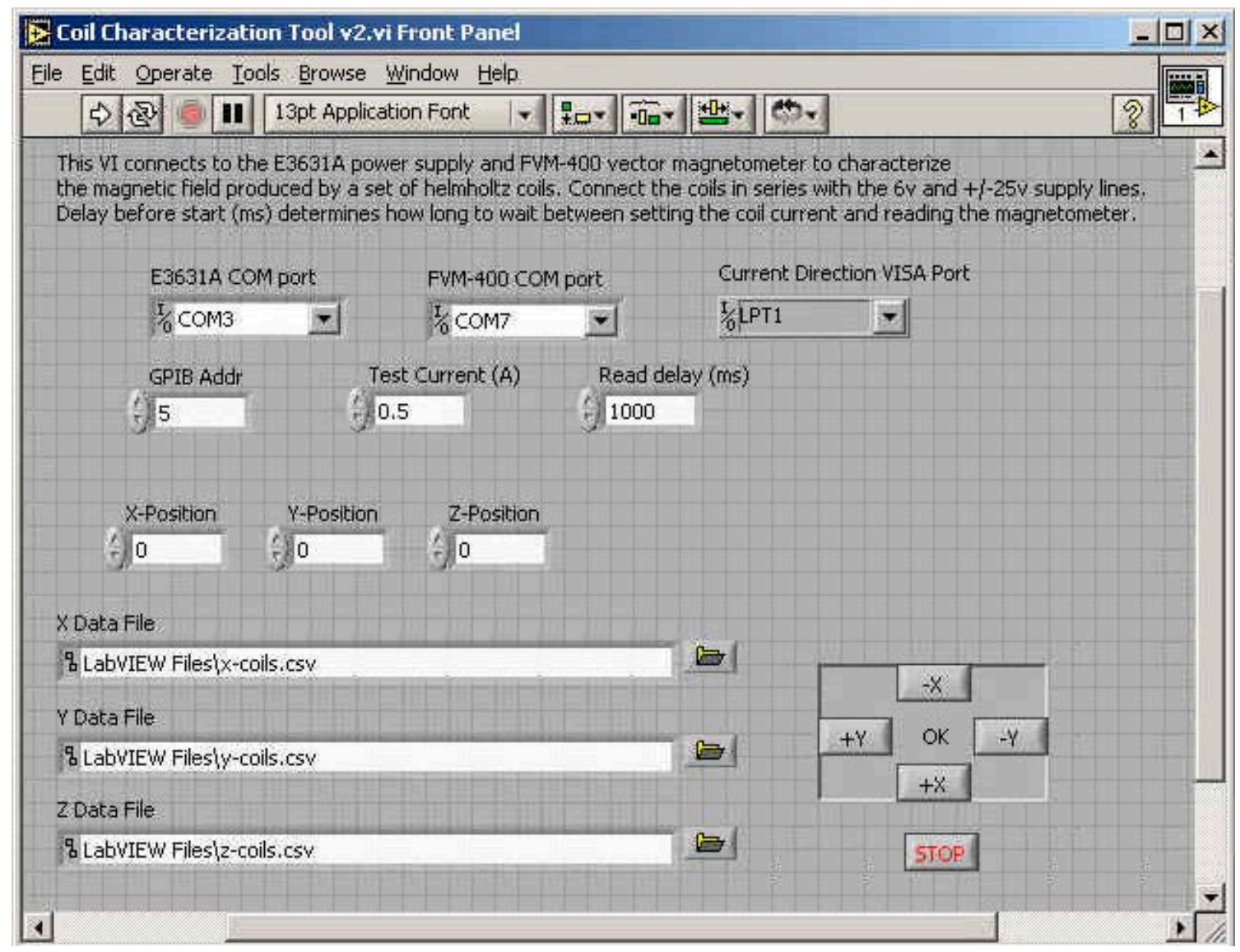

Coil Characterization Tool v2.vi is used to map the magnetic field inside the Helmholtz cage. The user places the magnetometer probe at a position on the grid paper, notes the coordinates, and clicks the appropriate direction on the bottom right. The directional buttons automatically convert the coordinate system of the magnetometer depending on how the magnetometer is oriented so the field values will always match the coil directions. This process is repeated for multiple data points and the resulting data is compiled into a CSV output file. 


\section{Calibration Procedures}

The following attachment contains the step-by-step procedures for performing magnetometer calibration using the hardware and software described by this paper. 


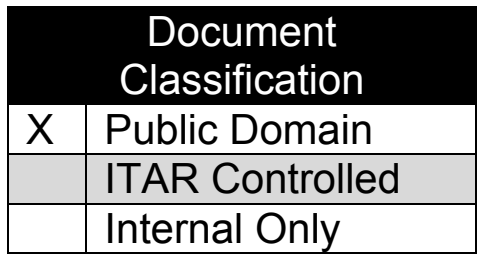

\section{PolySat Magnetometer Calibration Procedures (PMCP)}

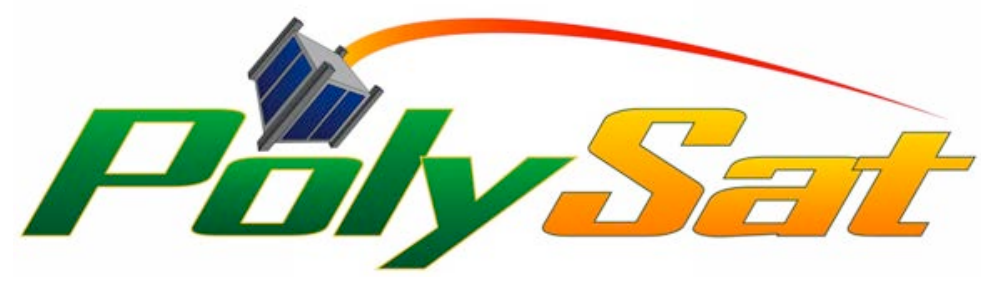

Document Number:

Project:

Date:

Satellite Name:

Satellite Serial Number:

Satellite Size:

Location:

Test Engineer:

QA Engineer:

Support Engineers: 
PolySat Magnetometer Calibration Procedures (PMCP)

\section{CHANGE HISTORY LOG}

\begin{tabular}{|l|l|l|l|}
\hline Date & Rev & Author & Description of Changes \\
\hline $11 / 22 / 12$ & Draft & Justin Foley & Initial draft \\
\hline $12 / 04 / 12$ & 1.0 & Justin Foley & Changed to standard procedures format \\
\hline & & & \\
\hline & & & \\
\hline & & & \\
\hline & & & \\
\hline & & & \\
\hline & & & \\
\hline
\end{tabular}




\section{Introduction}

This standard PolySat Magnetometer Calibration Procedure (PMCP) describes the process of determining the linear coefficients for individual magnetometers on a next generation PolySatbuilt CubeSat bus. In order to provide reliable magnetic measurements, the magnetometers on each CubeSat unit must be calibrated using well-known magnetic fields generated by a Helmholtz cage.

\section{Applicable Documents}

The following documents form a part of this document to the extent specified herein. In the event of conflict between the documents referenced herein and the contents of this document, the contents of this document shall take precedence.

PolySat Documents

SBOP

PolySat System Board Operating Procedures

\section{Required Equipment}

Gather all required equipment list below for performing this Fit Check.

- CubeSat bus w/ umbilical

- HMC5883L development board w/ Arduino

- Computer running LabVIEW

- E3631A Power Supply w/ GPIB to USB Adapter

- FVM-400 vector magnetometer kit

- Magnetometer test stand

- CubeSat bus test stand

- Polarity relay box w/ power supply

- Male to female DB-25 cable

- $6 \times$ short banana to banana leads

- Helmholtz cage

- Helmholtz cage interface cable (mini din to RJ-45)

- Digital Camera

- $\quad$ ESD wrist straps
QA Initials:

QA Initials:

QA Initials:

QA Initials:

QA Initials:

QA Initials:

QA Initials:

QA Initials:

QA Initials:

QA Initials:

QA Initials:

QA Initials:

QA Initials:

QA Initials: 


\section{Prepare the Test Setup}

\begin{tabular}{|c|c|c|c|}
\hline Step \# & Description & Time & Sig. \\
\hline 1. & $\begin{array}{l}\text { Connect all equipment } \\
\text { Set up computer on workbench. }\end{array}$ & & \\
\hline 2. & $\begin{array}{l}\text { Connect the E3631A power supply to the computer via GPIB to USB } \\
\text { adapter. }\end{array}$ & & \\
\hline 3. & $\begin{array}{l}\text { Connect the FVM- } 400 \text { vector magnetometer to its power adaptor and } \\
\text { its power adaptor to a wall outlet, connect the serial adaptor to the } \\
\text { computer's RS- } 232 \text { port. }\end{array}$ & & \\
\hline 4. & $\begin{array}{l}\text { Connect the FVM- } 400 \text { vector magnetometer probe to the hand unit } \\
\text { with the included RJ- } 45 \text { patch cable. } \\
\text { Note: Use only the included RJ- } 45 \text { patch cable for calibration } \\
\text { purposes. }\end{array}$ & & \\
\hline 5. & $\begin{array}{l}\text { Connect the polarity switching box to its power adapter, connect the } \\
\text { power adapter to a wall outlet (green power light should illuminate), } \\
\text { connect the polarity switching box to the computer's parallel port via } \\
\text { male to female DB- } 25 \text { cable. }\end{array}$ & & \\
\hline 6. & $\begin{array}{l}\text { Connect the polarity switching box to the } 3 \text { E3631A power supply } \\
\text { outputs via } 6 \text { banana to banana leads. }\end{array}$ & & \\
\hline 7. & $\begin{array}{l}\text { Set up the Helmholtz cage in a magnetically quiet location - it is OK } \\
\text { to have constant fields nearby as these will be subtracted out, } \\
\text { however large metal objects that move (carts, doors) may cause } \\
\text { interference. }\end{array}$ & & \\
\hline 8. & $\begin{array}{l}\text { Take photos of the test setup, concentrating on the electrical } \\
\text { connections and interfaces. }\end{array}$ & & \\
\hline 9. & $\begin{array}{l}\text { Power on the E3631A power supply, making note of the GPIB } \\
\text { address upon startup, write address below: } \\
\text { GPIB Address: }\end{array}$ & & \\
\hline
\end{tabular}




\begin{tabular}{|c|c|c|c|}
\hline Step \# & Description & Time & Sig. \\
\hline 10. & $\begin{array}{l}\text { Power on the FVM- } 400 \text { vector magnetometer and confirm expected } \\
\text { magnetic field readings for the area. Total magnitude should be on } \\
\text { the order of } 30,000 \text { to } 50,000 \mathrm{nT} \text {. }\end{array}$ & & \\
\hline 11. & $\begin{array}{l}\text { Press the REM button on the FVM- } 400 \text { hand unit to enable computer } \\
\text { access via the RS- } 232 \text { cable. }\end{array}$ & & \\
\hline 12. & Power on the computer. & & \\
\hline 13. & $\begin{array}{l}\text { Ensure all serial ports are properly assigned in the Device Manager. } \\
\text { Defaults used by the LabVIEW programs are: } \\
\text { - COM3 - GPIB Adapter "USB Serial Port" } \\
\text { - COM7 - FVM-400 Magnetometer "Prolific USB-to-Serial } \\
\text { Comm Port" }\end{array}$ & & \\
\hline 14. & $\begin{array}{l}\text { Perform Calibration Sweeps } \\
\text { If engineers intend to remain near to the Helmholtz cage during } \\
\text { testing, ensure all ferrous metal items and cell phones have been } \\
\text { removed from pockets and moved out of the area. Steel-toed shoes } \\
\text { and rolling office chairs are mobile and can also cause non-constant } \\
\text { interference. }\end{array}$ & & \\
\hline 15. & $\begin{array}{l}\text { Open "Coil Calibration Curve.vi", change COM ports or GPIB } \\
\text { address if necessary. }\end{array}$ & & \\
\hline 16. & Place the vector magnetometer probe on the magnetometer test stand. & & \\
\hline 17. & $\begin{array}{l}\text { Place vector magnetometer probe } \mathrm{YZ} \text { sensors at center point of } \\
\text { Helmholtz cage, matching the coordinate system of the probe to the } \\
\text { cage. Carefully align the axes as accurately as possible. }\end{array}$ & & \\
\hline 18. & Take photos of the probe placement within the Helmholtz cage. & & \\
\hline 19. & Select output file, save as "YZcalibration.csv" & & \\
\hline 20. & $\begin{array}{l}\text { Set delay before start if desired - this allows the engineers to clear } \\
\text { the space before the test starts if interference is expected as a result of } \\
\text { metal or cell phones in pockets. }\end{array}$ & & \\
\hline 21. & Press Run in top left corner and wait until finished. & & \\
\hline
\end{tabular}




\begin{tabular}{|c|c|c|c|}
\hline Step \# & $\begin{array}{ll}\text { Description } & \\
& \text { Date: }\end{array}$ & Time & Sig. \\
\hline 22. & $\begin{array}{l}\text { Move vector magnetometer so } \mathrm{X} \text { sensor is centered in the cage. Make } \\
\text { sure the axes are aligned as accurately as possible. }\end{array}$ & & \\
\hline 23. & Take photos of the probe placement within the Helmholtz cage. & & \\
\hline 24. & Change output file to "Xcalibration.csv" & & \\
\hline 25. & Press Run and wait until finished. & & \\
\hline 26. & Close LabVIEW. & & \\
\hline 27. & $\begin{array}{l}\text { Combine Calibration Constants } \\
\text { Open "Xcalibration.csv" and "YZcalibration.csv" in a text editor. }\end{array}$ & & \\
\hline 28. & Create a new text file called "XYZcalibration.csv" & & \\
\hline 29. & Copy line 1 of "Xcalibration.csv" to line 1 of "XYZcalibration.csv" & & \\
\hline 30. & $\begin{array}{l}\text { Copy lines } 2 \text { and } 3 \text { of "YZcalibration.csv" to lines } 2 \text { and } 3 \text { of } \\
\text { "XYZcalibration.csv" }\end{array}$ & & \\
\hline 31. & $\begin{array}{l}\text { Close and save "XYZcalibration.csv" } \\
\text { Use this calibration file for the rest of these procedures unless } \\
\text { adjustments are made to the test setup or surrounding environment } \\
\text { that might affect nearby magnetic fields. }\end{array}$ & & \\
\hline 32. & Notes: & & \\
\hline
\end{tabular}




\section{Calibrate a Standalone HMC5883L}

Note: The following procedures assume the Helmholtz cage has been calibrated according to Section 4.

\begin{tabular}{|c|c|c|c|}
\hline Step \# & Description & Time & Sig. \\
\hline 1. & Put on ESD wrist strap and connect to grounding point. & & \\
\hline 2. & $\begin{array}{l}\text { Connect the HMC5883L development board power and } \mathrm{i}^{2} \mathrm{c} \text { lines to } \\
\text { the Arduino board. }\end{array}$ & & \\
\hline 3. & Connect the Arduino board to the computer via USB. & & \\
\hline 4. & $\begin{array}{l}\text { Take photos of the test setup, concentrating on the electrical } \\
\text { connections and interfaces. }\end{array}$ & & \\
\hline 5. & $\begin{array}{l}\text { Ensure the Arduino serial port is properly set in the device manager. } \\
\text { The default used by the LabVIEW programs is COM4 } \\
\text { "Communications Port" } \\
\text { Note: If LabVIEW was not closed after the previous section, restart } \\
\text { LabVIEW for the new serial port to be recognized. }\end{array}$ & & \\
\hline 6. & $\begin{array}{l}\text { Place the HMC5883L development board on the magnetometer test } \\
\text { stand in the center of the Helmholtz cage, matching the coordinate } \\
\text { system of the board to the cage. Carefully align the axes as accurately } \\
\text { as possible. }\end{array}$ & & \\
\hline 7. & $\begin{array}{l}\text { Take photos of the development board placement within the } \\
\text { Helmholtz cage. }\end{array}$ & & \\
\hline 8. & $\begin{array}{l}\text { Open "Field sim and mag reader.vi", change the COM ports or GPIB } \\
\text { address if necessary, and change the FVM/HMC switch to HMC. }\end{array}$ & & \\
\hline 9. & $\begin{array}{l}\text { Select an appropriate Mag File to test the } \mathrm{X} \text { and } \mathrm{Y} \text { axes of the } \\
\text { HMC5883L, such as "Rotate about } \mathrm{Z} \text { at } 30000 \mathrm{nT} \times 5 . \mathrm{csv} \text { ", write the } \\
\text { filename below: } \\
\text { Mag File: }\end{array}$ & & \\
\hline 10. & Select Calibration File "XYZcalibration.csv" & & \\
\hline
\end{tabular}




\begin{tabular}{|c|c|c|c|}
\hline Step \# & Description & Time & Sig. \\
\hline 11. & $\begin{array}{l}\text { Select Output File with an appropriate name, i.e. " } \mathrm{Z} \text { spin at } 30000 \mathrm{nT} \\
\text { - standalone chip.csv", write the filename below: } \\
\text { Output File: }\end{array}$ & & \\
\hline 12. & Click Run and wait to finish. & & \\
\hline 13. & $\begin{array}{l}\text { Select an appropriate Mag File to test the } \mathrm{Y} \text { and } \mathrm{Z} \text { axes of the } \\
\text { HMC } 5883 \mathrm{~L} \text {, such as "Rotate about } \mathrm{X} \text { at } 30000 \mathrm{nT} \times 5 . \mathrm{csv} \text { ", write the } \\
\text { filename below: } \\
\text { Mag File: }\end{array}$ & & \\
\hline 14. & Select Calibration File "XYZcalibration.csv" & & \\
\hline 15. & $\begin{array}{l}\text { Select Output File with an appropriate name, i.e. "X spin at } 30000 \mathrm{nT} \\
\text { - standalone chip.csv", write the filename below: } \\
\text { Output File: }\end{array}$ & & \\
\hline 16. & Click Run and wait to finish. & & \\
\hline 17. & Close LabVIEW. & & \\
\hline 18. & Open the resulting Output Files in Excel. & & \\
\hline 19. & $\begin{array}{l}\text { For each magnetometer axis tested, create a new Scatter Plot chart } \\
\text { using the HMC5883L values on the X-axis and actual magnetic field } \\
\text { values on the Y-axis. }\end{array}$ & & \\
\hline 20. & $\begin{array}{l}\text { Right click on the data series in each charts and add linear trendlines. } \\
\text { In the trendline menu, check the boxes to display the equation and } \mathrm{R}^{2} \\
\text { values on the chart. }\end{array}$ & & \\
\hline
\end{tabular}




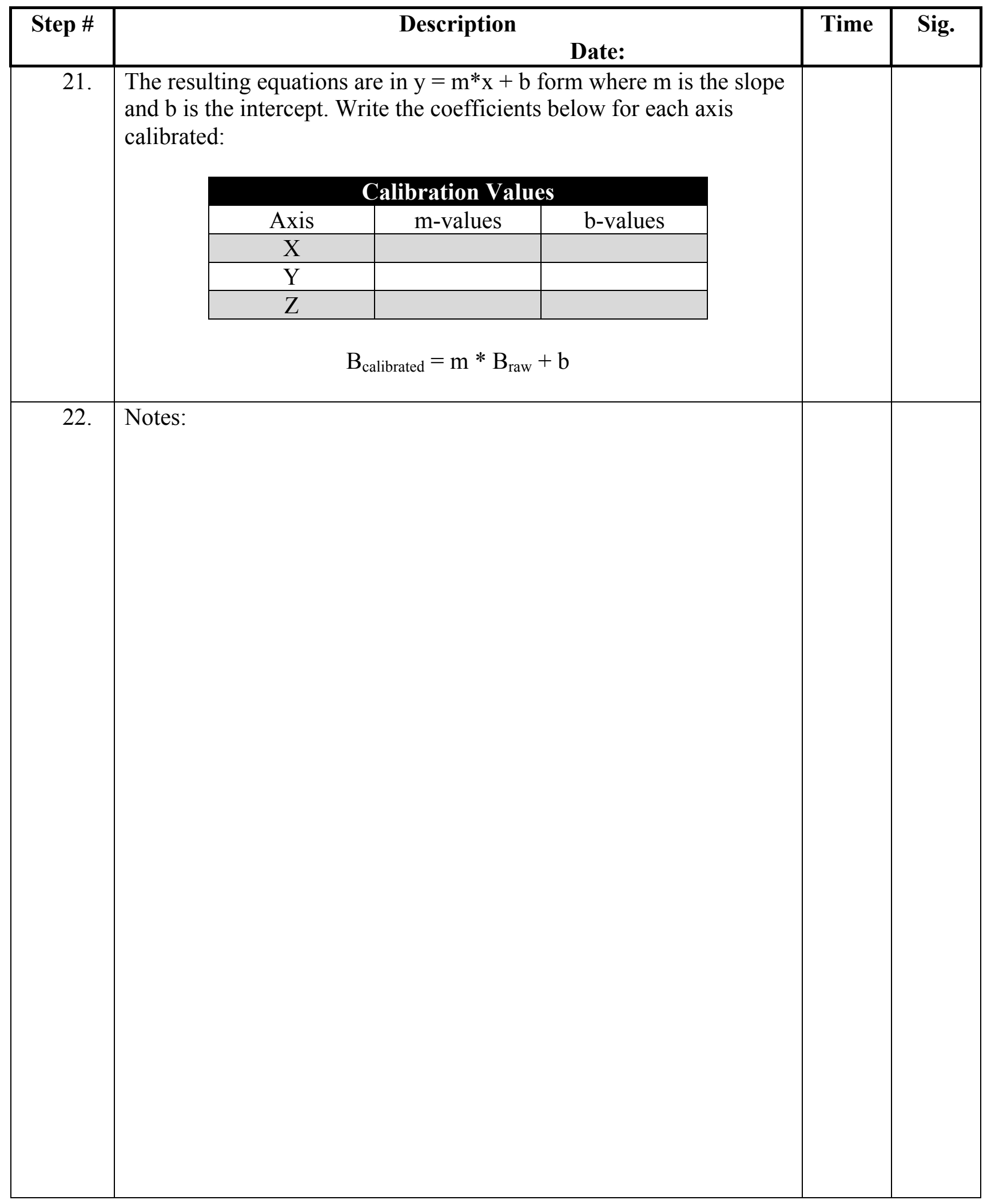




\section{Calibrate a CubeSat Bus}

Note: The following procedures assume the Helmholtz cage has been calibrated according to Section 4.

\begin{tabular}{|c|c|c|c|}
\hline Step \# & Description & Time & Sig. \\
\hline 1. & Put on ESD wrist strap and connect to grounding point. & & \\
\hline 2. & $\begin{array}{l}\text { Place the CubeSat on the CubeSat bus test stand in the center of the } \\
\text { Helmholtz cage, matching the coordinate system of the bus to the } \\
\text { cage. Carefully align the axes as accurately as possible. }\end{array}$ & & \\
\hline 3. & Take photos of the CubeSat placement within the Helmholtz cage. & & \\
\hline 4. & $\begin{array}{l}\text { Connect the CubeSat to the umbilical, the umbilical to the computer, } \\
\text { and prepare a terminal according to the system board operation } \\
\text { procedures document. }\end{array}$ & & \\
\hline 5. & $\begin{array}{l}\text { Take photos of the test setup, concentrating on the electrical } \\
\text { connections and interfaces. }\end{array}$ & & \\
\hline 6. & Open "Set Mag Field Vector.vi" & & \\
\hline 7. & Set the E3631A COM port and GPIB address if necessary. & & \\
\hline 8. & Select Calibration File "XYZcalibration.csv" & & \\
\hline 9. & Press "Local" on the E3631A, then press "Output" to turn on. & & \\
\hline 10. & $\begin{array}{l}\text { In "Set Mag Field Vector.vi", leave X, Y and Z components at } 0 \text {, and } \\
\text { press Run to set the magnetic field. If the E3631A successfully set } \\
\text { output currents, move on to the next step. }\end{array}$ & & \\
\hline 11. & $\begin{array}{l}\text { In the terminal window, access the magnetometer readings according } \\
\text { to the system board operation procedures document. }\end{array}$ & & \\
\hline
\end{tabular}




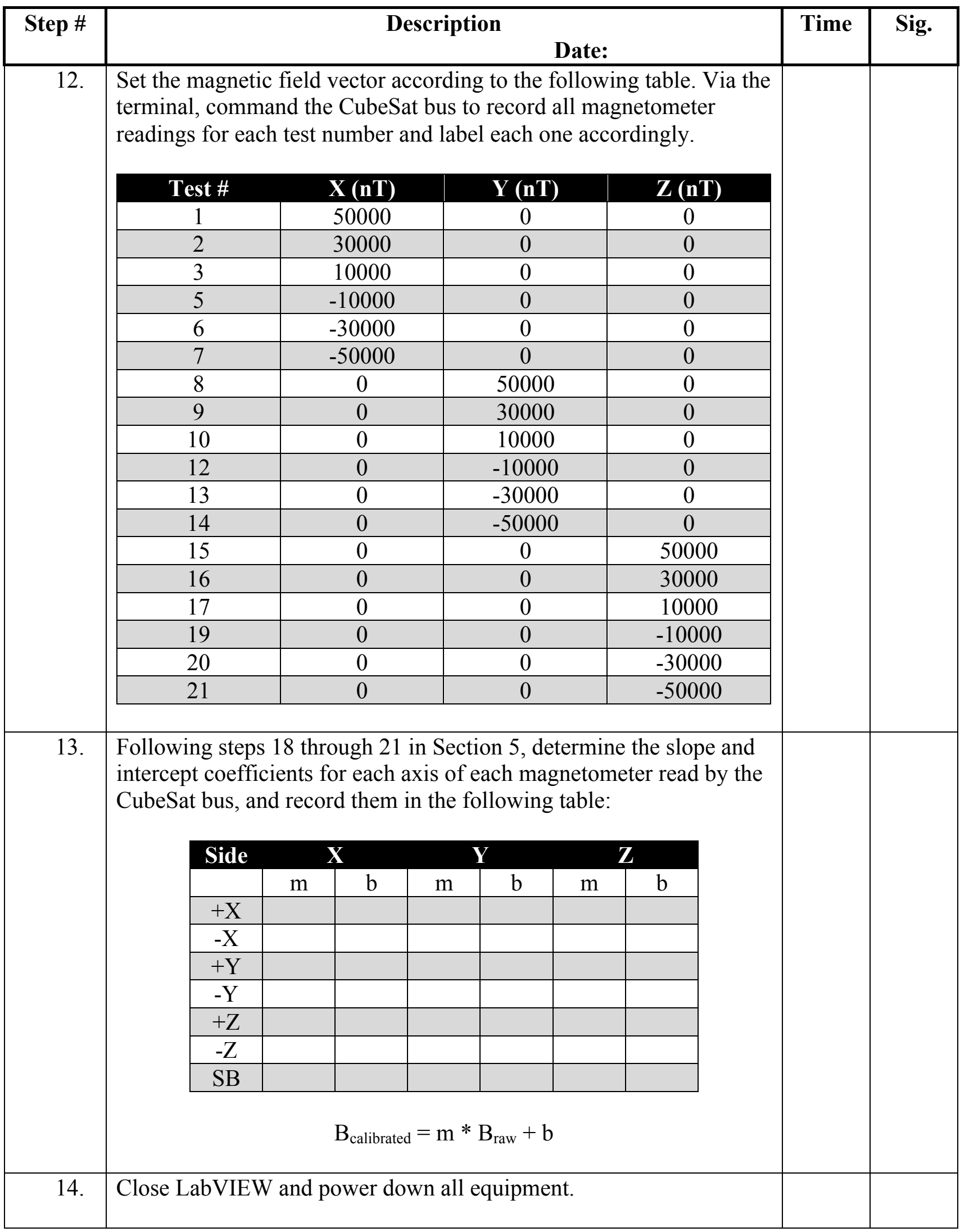


PolySat Magnetometer Calibration Procedures (PMCP)

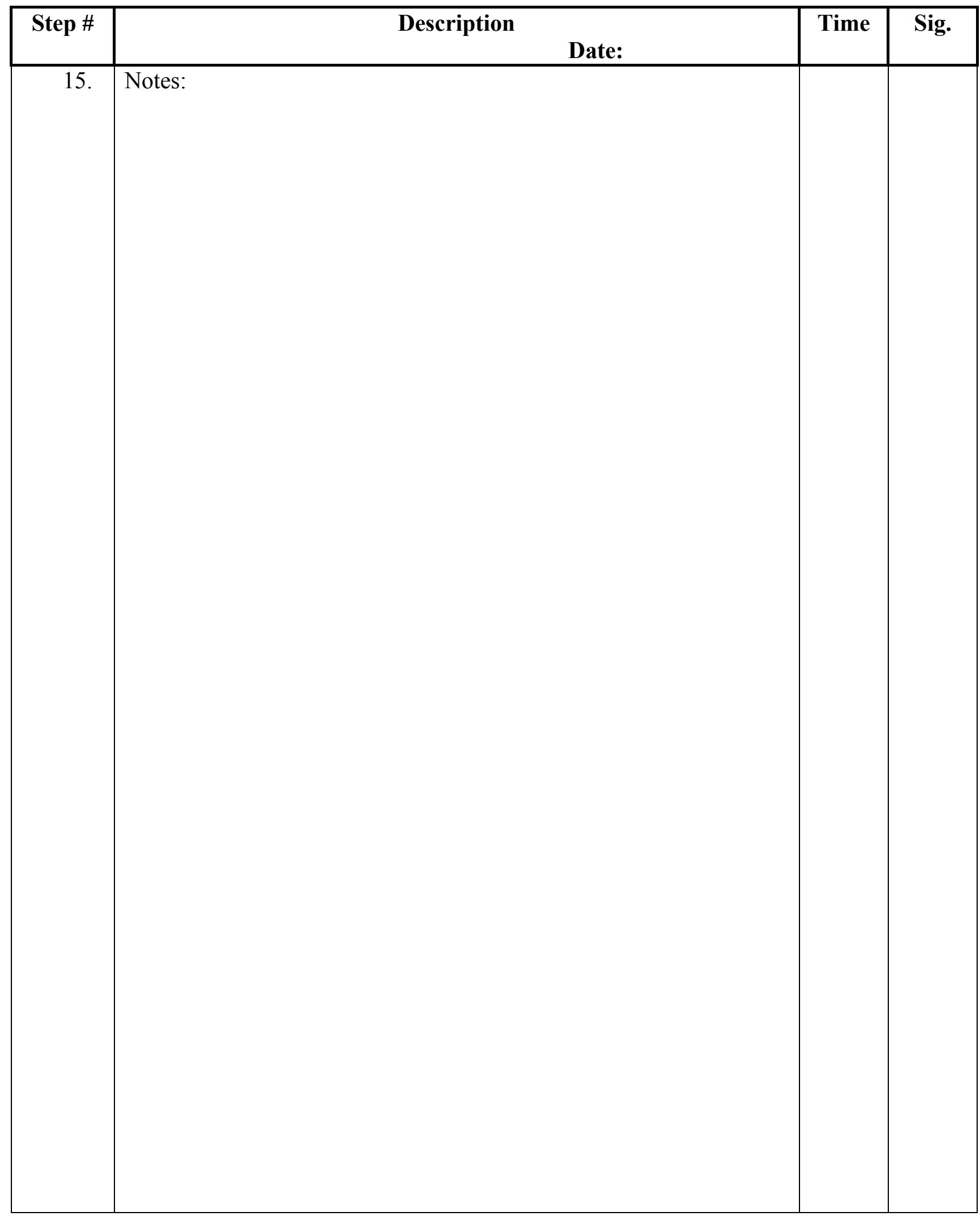

